\title{
NBSIR 75-941
}

\section{The National Measurement System for Far Ultraviolet Radiometry}

W. R. Ott

Institute for Basic Standards

National Bureau of Standards

Washington, D.C. 20234

June 1977

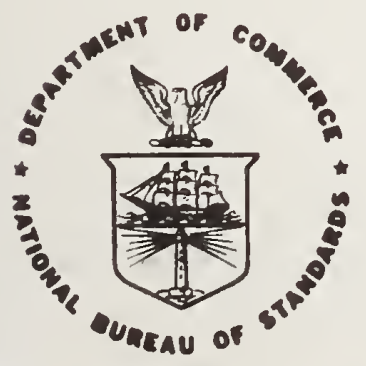

QC U. S. DEPARTMENT OF COMMERCE

100 NATIONAL BUREAU OF BTANDARDS

.456

\#5-941

1977 


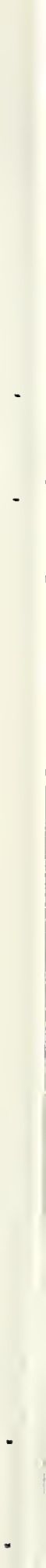


NBSIR 75-941

THE NATIONAL MEASUREMENT SYSTEM FOR FAR ULTRAVIOLET RADIOMETRY

W. R. Ott

Institute for Basic Standards

National Bureau of Standards

Washington, D.C. 20234

June 1977

U.8. DEPARTMENT OF COMMERCE, Juanita M. Kreps, Secrotery

Dr. Bidney Harman, Under Sacrotery

Jordan J. Baruch, Assistant Socrotary for Scionce and Tochnology

NATIONAL BUREAU OF STANDARDS, Emeat Ambler, Acting Director 
EXECUTIVE SUMMARY . . . . . . . . . . . . . . . . . . . . . . . 1

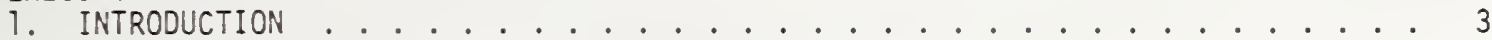

2. STRUCTURE OF THE MEASUREMENT SYSTEM . . . . . . . . . . . . . . . . . 4

2.1 Conceptual System . . . . . . . . . . . . . 4

2.2 Basic Technical Infrastructure . . . . . . . . . . . . . 5

2.2.1 Documentary Specification System . . . . . . . . . . 5

2.2.1.1 Standardization Institutions............ 5

2.2.1.2 Survey of Documentary Standards . . . . . . . . 6

2.2.2 Instrumentation System . . . . . . . . . . . . 7

2.2.2.1 Measurement Tools and Techniques . . . . . . . . 7

2.2.2.1.1 Standard Sources ........... . 7

2.2.2.1.2 Standard Detectors .......... 9

2.2.2.2 The Instrumentation Industry . . . . . . . . 10

2.2.3 Reference Data ................ . . 12

2.2.4 Reference Material ............... . . 12

2.2.5 Science and People ................. . . 13

2.3 Realized Measurement Capabilities . . . . . . . . . . . . 13

2.4 Dissemination and Enforcement Network . . . . . . . . . . . 15

2.4.1 Central Standards Authorities . . . . . . . . . . . 15

2.4.2 State and Local Offices of Weights and Measures . . . . . . . . 17

2.4.3 Standards and Testing Laboratories and Services . . . . . . . 17

2.4 .4 Regulatory Agencies.............. . . . 17

2.5 Direct Measurements . . . . . . . . . . . . . . . . 20

2.5.1 Analysis of Suppliers and Users . . . . . . . . . . . 20

2.5.2 Highlights re Major Users . . . . . . . . . . . . . 20

3. IMPACT, STATUS, AND TRENDS OF MEASUREMENT SYSTEM . . . . . . . . . . . 23

3.1 Impact of Measurements . . . . . . . . . . . . . . . . . 23

3.1.1 Functiona1, Technological, and Scientific Applications . . . . 24

3.1.1.1 Photochemistry . . . . . . . . . . . . . 24

3.1.1.2 Bacteriological ............... 26

3.1.1.3 Environmental Studies . . . . . . . . . . . . 27

3.1.1.4 Medical and Therapeutic ............ . . 28

3.1.1.5 National Fusion Research ............ 30

3.1.1.6 Space Sciences ... . . . . . . . . . . . . 30

3.1 .1 .7 Vacuum U1traviolet and X-Ray Lasers . . . . . . . . 32

3.1.1.8 Photobiology .............. . . 33

3.1.1.9 Plasma Chemistry . . . . . . . . . . . . . 34

3.1.2 Economic Impacts--Costs and Benefits . . . . . . . . . . . 35

3.1.3 Social, Human, Person-on-the-Street Impacts . . . . . . . . 36

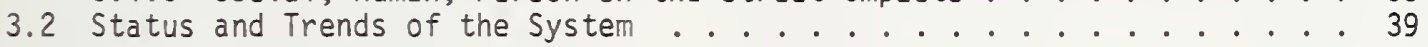

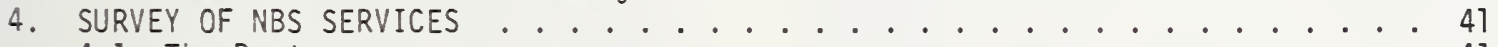

4.1 The Past . . . . . . . . . . . . . . . . 41

4.2 The Present--Scope of NBS Services............... 41

4.2.1 Description of NBS Services.................... 41

4.2.2 Users of NBS Services................. . . . 44

4.2.3 Alternate Sources . . . . . . . . . . . . . . 45

4.2.4 Funding Sources for NBS Services . . . . . . . . . . . . 46

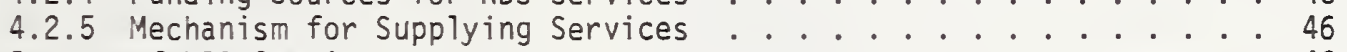

4.3 Impact of NBS Services................ . . . . 46

4.3.1 Economic Impact of Major User Classes . . . . . . . . . . 46

4.3.2 Technological Impact of Services . . . . . . . . . . . 49

4.3.3 Payoff from Changes in IIBS Services............ . 49

4.4 Evaluation of NBS Program ...................... 50

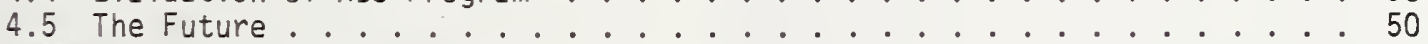

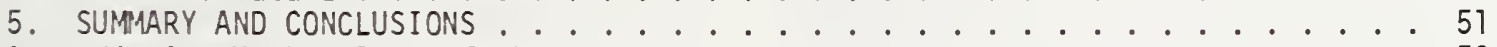

Appendix A. Methodology of the Study . . . . . . . . . . . . . . . 52

Appendix B. A Partial Listing of Manufacturers and Distributors of Incoherent

Appendix C. An International List of Companies Having an Interest in Plasma

Chemistry Applications ................ 56 


\section{LIST OF FIGURES}

Figure 1. The ultraviolet region of the electromagnetic spectrum defined in terms of wavelength $(\mathrm{nm})$, energy $(\mathrm{eV})$, and some key processes .... 3

Figure 2. Primary and transfer standard sources which have been applied in the ultraviolet . . . . . . . . . . . . . . . . . 7

Figure 3. Comparison of the spectral radiance of several ultraviolet standard sources at typical operating conditions ............. 8

Figure 4. Primary and transfer standard detectors which have been applied in the ultraviolet . . . . . . . . . . . . . . . . 8

Figure 5. A copy of a publicity release illustrating a recent NBS cooperative effort with the instrumentation industry . . . . . . . . . . 18

Figure 6. The ultraviolet radiation exposure standard recommended by the National Institute for 0ccupational Safety and Health is illustrated in terms of the relative spectral effectiveness in producing a standard erythemal response in the skin and eyes.......... . 18

Figure 7. A copy of a magazine advertisement illustrating a technology forecast with respect to laser fusion systems . . . . . . . . . . . 40

Figure 8. Current and projected NBS standard source and detector capabilities. The dashed lines indicate near term projections . . . . . . . . 42

\section{LIST OF TABLES}

Table 1. The value of shipments of a variety of ultraviolet emitting products for three different years according to the U.S. Census of Manufacturers .................. . . 10

Table 2. An overview of the far ultraviolet radiometric needs and capabilities of the National Measurement System .............. . 14

Table 3. The Far U1traviolet Radiometry National Measurement System illustrated as a direct transactions input-output matrix . . . . . . . 21

Table 4. List of users described in section 3.1.1 together with applicable Standard Industrial Classification Codes (SIC-1972) . . . . . . . . . 24

Table 5. Occupations potentially associated with ultraviolet radiation exposures ...................... 38

Table 6. List of customers requiring NBS ultraviolet radiometric services

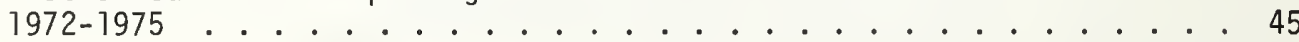


on a study of

THE NATIONAL MEASUREMENT SYSTEM FOR FAR ULTRAVIOLET RADIOMETRY

William R. Ott

NBS Institute for Basic Standards

\section{EXECUTIVE SUMMARY}

Radiometry is the quantitative measurement of radiant power. By restricting the region of interest to the far ultraviolet, the wavelength region from $10 \mathrm{~nm}$ to $300 \mathrm{~nm}$ is specified. The corresponding photon energy range extends from $125 \mathrm{eV}$ to $4 \mathrm{eV}$, very large compared for example with the visible range which extends only from $3 \mathrm{eV}$ to about $1.8 \mathrm{eV}$. Because of this, a wide variety of phenomena and radiometric applications can be found throughout the far ultraviolet. This report is a description of the evolving National Measurement System in the field of Far U1traviolet Radiometry; at the same time a critical evaluation of the NBS role in the System is made in order that we might optimize our efforts toward serving the System and thus promote progress toward the national goals of our government.

The technological importance of the far ultraviolet spectral region stems essentially from: (a) the increasing use of high temperature devices such as the fusion plasmas being produced in the search for new energy sources; (b) the industrial potential of far uitraviolet and $x$-ray lasers; (c) the high energy content of short wavelength radiation with the consequent ability to drive and control chemical reactions; and (d) the very low diffraction limit of short wavelength radiation. The problem areas where ultraviolet radiation measurements are important include both national research and development programs and major industrial applications in the following fields:
(1) Industrial PHotochemistry
(2) Bacteriological Control
(3) Environmental Studies
(4) Medical Therapy
(5) Fusion Research
(6) Space Science
(7) UTtraviolet and X-Ray Lasers
(8) Plasma Chemistry
(9) Photobiology

In order to evaluate whether or not an ultraviolet radiation measurement capability is in the national interest and affects our well being and the common good, both the social and economic significance of those activities which depend upon such a capability are discussed. Examples of areas of impact where the ultraviolet measurement system enjoys considerable social and economic leverage are national health and safety and solar simulation.

Numerous studies which are cited in this microstudy indicate that ultraviolet radiation can be dangerous and can cause such things as incapacitating burns (sunlamps and arc welders are the biggest culprits), premature wrinkling, and skin cancer. In view of the increasing use of ultraviolet lamps in our society for illumination, advertising, bacteriological control, medical treatments and industrial polymerization applications, and in view of our recent concern with the disastrous consequences which would result from even a small increase in solar ultraviolet transmission through the atmosphere, a measurement capability is absolutely necessary. It is used not only to monitor the light environment and thus guarantee radiation safety but also to enable definitive experiments which will accurately specify the safety limits themselves. If the social benefit is obvious, the economic leverage is no less so. Our work force is weakened considerably not only by work-related radiation accidents involving arc welders, high intensity sterilization lamps, etc., but also by the common "weekend-in-the-garden" sunburn. Outdoor workers are especially affected and experience in addition a significantly high incidence of skin cancer. The statistics are available and one can easily estimate the economic impact of thousands of careers terminated in this way.

Accelerated weathering machines and solar simulators are used by the paint, dye, plastics, and building material industries as well as for space-related applications, for example, in determining the lifetime of solar cell arrays. Correlations up to now have not always been significant partly because the irradiance is specified by time exposed and not in terms of intensity and spectral distribution. Because of the uncertainties in the lamp characteristics, not only are large 
amounts of money and effort wasted annually in the collection of misinformation and in some cases by the production and distribution of inferior products, but also the industries are discouraged from increasing their efforts toward developing superior weather-resistant products. Ultraviolet calibrations of these machines for wavelengths as $10 \mathrm{w}$ as $121.6 \mathrm{~nm}$ are required if accuracy, consistency, and cost effectiveness are to be achieved in testing. Manufacturers of the simulators are equally concerned with improved diagnostic methods since their world market could be threatened by the imposition of radiometric specifications on the machines by international standardizing bodies.

The NBS program on far ultraviolet radiometric calibrations is carried out mainly in the Plasma Spectroscopy Section (232.07) and in the Far UV Physics Section (232.03). It consists of several complementary components and makes use of absolute detectors as well as standard radiation sources. The primary consumers of our far ultraviolet services have been the space scientists who need absolute detectors and sources to determine the response of spectrometric optical systems. At present these users dominate by about 10:1 over the combined number of all other consumers. This statistic is due mainly to the chronology of how the National Measurement System has evolved: research and scientific explorations (NASA in the 60's) force the development of advanced technology which, in turn, stimulates and makes possible new applications of that technology. There is substantial evidence that we are entering this latter productive and potentially explosive stage. Nevertheless, it is shown in this report that there is little systematic interaction between the standards community and the users groups. Aware of this evolution and the changing priorities, NBS cannot only be open and receptive as it has always been, but must actively develop new contacts. The secondary standards laboratories and the instrumentation industry are two sectors of the measurement system with which NBS could achieve a high leverage impact through an expanded standards program and improved communications. It is also recommended that NBS initiate more mini-workshops similar to the successful meetings involving several regulatory agencies, which we organized during 1972-1974. Photochemical and biomedical applications should be the first areas where NBS should generate such a new initiative.

There are several technical recommendations in this report which are the result of some preliminary contacts of the type just discussed.
(1) NBS, although boasting two complementary programs toward the development of primary, far ultraviolet radiometric source standards, currently supports no efforts to develop reliable transfer standards. This situation must be rectified in view of requirements for field uncertainties of about 1 percent between $200 \mathrm{~nm}$ and $300 \mathrm{~nm}$, 5 percent between $90 \mathrm{~nm}$ and $200 \mathrm{~nm}$, and 10 percent below $90 \mathrm{~nm}$.

(2) We must plan to develop ultraviolet source standards of spectral irradiance, the unit of principal interest to the users. Unless this is done, we must leave it up to the individual users to generate their own irradiance standards by way of our spectral radiance standards. Not only is this an expensive and counter-productive way to go, but also it makes for a very uncertain traceability to NBS.

(3) The calibration limits for NBS ultraviolet standard detectors must be extended toward both shorter and longer wavelengths to fully cover the range $5 \mathrm{~nm}$ to $400 \mathrm{~nm}$. Only then will several high priority users be adequately served. Uncertainties must be lowered to the 1-5 percent level, at least for wavelengths above $115 \mathrm{~nm}$.

(4) A new NBS service should be initiated which would provide for monochromator or filter transmission calibrations in the far ultraviolet.

(5) Internal consistency among the various NBS radiometric standards must be systematically checked. This includes such independent standards as photodiode detectors, synchrotron radiation, hydrogen àrc radiation, electrically calibrated radiometers, and tungsten lamps. 


\section{INTRODUCTION}

The need for an ultraviolet radiation measurement system may seem a bit remote to the average person on the street. This first reaction comes about because ultraviolet radiation, unlike visible radiation, is something that cannot be seen. However, just because it cannot be seen doesn't mean that this radiation is not useful. Radio waves, also invisible electromagnetic radiation, are an example of how we can extend our experiences beyond that which is obvious in nature. But whereas radio or long wave radiation is distinquished by its small interaction with atoms and molecules, ultraviolet or short wave radiation is distinguished by its very strong interaction.

The sensitivity of the human eye extends from the red $(700 \mathrm{~nm})$ to the blue $(400 \mathrm{~nm})$. It is thought to be an evolutionary characteristic of land animals that they did not develop the capacity to see beyond blue, or "ultraviolet" as it is called. The earth was unlivable in the beginning: intense and energetic ultraviolet radiation from the sun heated the surface and caused continuous photochemical and evolutionary reactions between the element and the primeval atmosphere. Because this radiation was deadly, life developed under the protective blanket of the sea. It was a necessary condition, before the first land animal could step out of the sea millions of years ago, that an oxygen $\left(\mathrm{O}_{2}\right)$ atmosphere had to evolve, which together with ozone $\left(\mathrm{O}_{3}\right)$ would effectively block out the energetic solar radiation below $280 \mathrm{~nm}$.

The ultraviolet wavelength region can be considered to extend from $400 \mathrm{~nm}$, the near ultraviolet, to about $4 \mathrm{~nm}$ where the soft $x$-ray region begins.* For perspective some key wavelengths in this region are illustrated in figure 1. The critical atmospheric ozone absorption occurs between 200-280 $\mathrm{nm}$. Molecular oxygen absorption characterizes the region below $200 \mathrm{~nm}$ and thus the terminology "vacuum ultraviolet" to indicate the necessity for the absence of air in a system designed to measure or utilize such radiation. Atomic hydrogen absorption sets in at $100 \mathrm{~nm}$ and sets a limit to the range of interstellar astrophysical measurements. However, the real importance of the ultraviolet wavelength region is contained in the energy scale corresponding to the wavelength scale shown in figure 1. The ultraviolet energy range spans from $3 \mathrm{eV}$ to $300 \mathrm{eV}$, which is quite large as compared to the visible for example, which extends only from

*1 nanometer $(\mathrm{nm})=10^{-9}$ meter $=10$ Angstrom
$1.5 \mathrm{eV}$ to $3 \mathrm{eV}$. This energetic radiation can be used to drive and control all sorts of chemical and biological reactions. For example extreme doses of radiation having energy between 3 and $6 \mathrm{eV}$ can cause skin cancer; controlled doses of the same radiation can heal. Continuous exposure to solar ultraviolet radiation causes the fading of paints and dyes; controlled exposures to artificial ultraviolet sources can be used to produce protective coatings on materials.

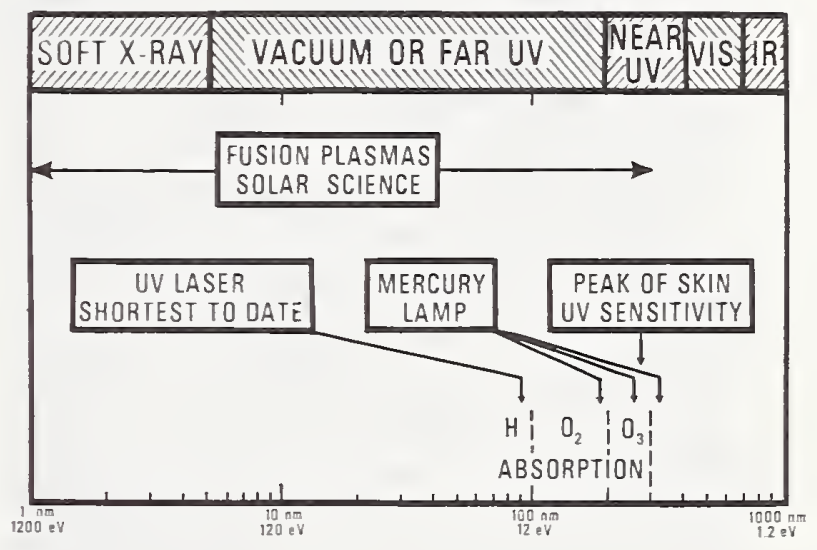

Figure 1. The ultraviolet region of the electromagnetic spectrum defined in terms of wavelength $(\mathrm{nm})$, energy $(\mathrm{eV})$ and some key processes.

The space science of the 60's and 70's literally reached beyond the atmosphere and demanded sophisticated ultraviolet instrumentation. The resulting technology, involving the production, detection, and analysis of ultraviolet radiation, is just now beginning to "spin-off" and show heavy impact in other areas.

This document describes the evolving National Measurement System in the field of U1traviolet Radiometry as seen from NBS. The term, National Measurement System, is a conceptual shorthand for the network of measurement standards and techniques, standardizing laboratories, quality control laboratories, and measuring operations of all kinds extending right to the ordinary citizen in his everyday life. It is important to periodically evaluate the role of the National Bureau of Standards (NBS) in the System in order that we may make best use of the System in promoting progress toward the national goals of our Government. Accordingly, we seek answers to these kinds of questions:

(1) Who makes ultraviolet radiation measurements, who are the users of NBS 
services, and what is the significance of their measurements?

(2) What are the interfaces of NBS with industry, instrument manufacturers, government agencies, calibration laboratories, state and local authorities, and trade and professional associations?

(3) What is the present capability of NBS in ultraviolet radiometry?

(4) What are the systems and instruments by which practical measurements are actually made, and what is known of their characteristics?

(5) Under what conditions is NBS accuracy usable by its customers; what are the service requirements?

(6) What important deficiencies exist and what areas should be emphasized in future planning?

This study on Far Untraviolet Radiometry is organized into many logical subsections some of which contain data and information appearing also in other subsections. This duplication is justified since it is expected that a reader will choose to survey only those sections relevant to his own interests. Therefore every effort has been made to make each section complete without resorting to tedious internal references. This study is undertaken with the assumption that NBS is expected not to perform all the many calibrations required by government and industry, but rather to continualzy monitor the measurement system, and on the basis of its findings, to undertake those calibrations and other tasks that will make the system function most effectively and economicarzy.

\section{STRUCTURE OF THE MEASUREMENT SYSTEM}

\subsection{Conceptual System}

The present scientific SI unit for the quantitative measurement of radiant energy is the watt as defined internationally in the Treaty of the Meter. The watt is a derived unit equal to one joule per second or

$$
\begin{aligned}
1 \text { watt } & =1 \text { joule } \mathrm{sec}^{-1} \\
& =1 \text { newton meter } \mathrm{sec}^{-1} \\
& =1 \mathrm{kilogram} \text { (meter) } \mathrm{sec}^{-3}
\end{aligned}
$$

Radiant energy, as defined in SI units, is therefore based on mechanical equivalents; how one best determines in a practical way a standard watt of radiant energy is still an open question at the present time.

Traditionally, the problem has been approached by the definition of an ideal blackbody radiator at a particular temperature T, for example, the temperature of the melting point of gold. In this case the spectral radiance, or the radiant energy per unit area, wavelength $(\lambda)$, and solid angle, in units of Watts $\mathrm{m}^{-2} \mathrm{~nm}^{-1} \mathrm{sr}^{-1}$, can be realized by applying Planck's blackbody radiation law

$$
L_{\lambda}=\frac{c_{1}}{\lambda^{5} \exp \left[c_{2}(\lambda T)^{-1}-1\right]}
$$

where $c=1.19096 \times 10^{-16} \mathrm{~W} \mathrm{~m}^{2} \mathrm{sr}^{-1}$ and where $c_{2}=1.4388 \times 10^{-2} \mathrm{~m}-\mathrm{K}$. Conventional blackbody cavities and radiators emit most of their light in the visible and infrared region of the spectrum. In order to shift the spectral distribution of a blackbody radiator into the ultraviolet, the temperature must be increased to such a large extent that solid blackbodies are no longer feas ible and plasma blackbodies would need to be utilized instead. These are generally very complicated devices requiring gas temperatures above $6000 \mathrm{~K}$ and pressures on the order of $10^{7} \mathrm{~N} \mathrm{~m}^{-2}$ (100 standard atmospheres). Nevertheless, several laboratories outside of the United States have been active in developing both continuous and pulsed plasma sources which appear to be capable of providing blackbody continuum radiation over at least a small portion of the ultraviolet spectral range.

There are several alternatives to the blackbody approach which are currently being investigated. For example, an electrically calibrated radiometer has been developed both at NBS and in Australia. With this technique one compares the amount of electrical energy needed to cause the same heating effect as a given amount of radiant energy. A second alternative is to measure the wavelength, $\lambda$, of the incident radiation and to determine the flux of photons, $N$, with an absolute detector. Highly energetic vacuum ultraviolet radiation can be depended upon to produce one electron per incident photon in a properly designed ionization chamber. The spectral irradiance is then given by $E_{\lambda}=N h c \lambda^{-1}$ where $h$ is Planck's constant and $c$ is the speed of light. A third method is to depend upon the calculated intensity of synchrotron radiation (dependent on electron energy, current, and radius of orbit) or the calculated intensity of a completely ionized equilibrated hydrogen arc plasma (dependent only on the condition of local thermodynamic equilibrium). More details on these various methods of establishing a radiometric base are contained in sections 2.2.2 and 4.2.1.

There are in general two ways to determine the radiant power of an unknown light source: (a) through the use of standard sources and (b) through the use of standard detectors. Standard sources are most useful when it is desired to know the emission 
characteristics of an unknown source. Standard detectors are most useful, on the other hand, when it is desired to know the photon or energy flux at the location of the detector. For example, if the quantity of interest is spectral radiance [watts $\mathrm{m}^{-2} \mathrm{~nm}^{-1} \mathrm{sr}^{-1}$ ] or the energy radiated per unit wavelength band $(\mathrm{nm})$ per unit solid angle $(s r)$, then the light source to be investigated as well as the standard source may be set up in such a manner that the radiation from both passes through the same optical-spectrometric arrangement. In this way all geometric and other specific factors of the instrumentation are eliminated. In short the calibration is effected by a simple substitution of sources in the experimental arrangement. On the other hand, if the quantity of interest is spectral irradiance [watts $\mathrm{m}^{-2} \mathrm{~nm}^{-1}$ ] or the energy incident on a specified surface area $\left(\mathrm{m}^{2}\right)$ per unit wavelength band $(\mathrm{nm})$, a standard detector and filter arrangement may be placed at the location of the irradiated surface and the light source calibrated accordingly. Alternatively, if such a standard radiometer is not available, a standard source of spectral irradiance may be used in conjunction with a suitable diffusing element (to account for variations in the geometries of the standard and the unknown sources) to determine the response function of the user's diffuserradiometer system.

This microstudy is intended to determine the needs and requirements of the National Measurement System for measurements of this type but only in the far ultraviolet region of the spectrum, i.e., for wavelengths less than about $300 \mathrm{~nm}$. The region above $300 \mathrm{~nm}$, i.e., the near ultraviolet, visible, and infrared, is treated in another microstudy, Radiometry and Photometry, organized by Dr. Henry Kostkowski of the NBS Optical Physics Division. The scope of the two microstudies can be expected to overlap slightly in the near ultraviolet since the NBS far ultraviolet research efforts, in view of the traditional basis of radiometry, the

blackbody emitter, attempt to overlap into the wavelength range of conventional radiometry wherever possible in order to insure a consistent radiometric base.

\subsection{Basic Technical Infrastructure}

This section is intended to identify the various technical elements needed for realizing the conceptual system described in section 2.1. A more detailed description of those particular elements which have a rather direct impact on the user is contained in section 2.4, which addresses the nature of the dissemination and enforcement network.

\subsubsection{Documentary Specification System}

\subsubsection{Standardization Institutions}

Standardization institutions assist in transferring measurement technology from the research and development community to the user and act as an advisor to the central agencies regarding new and better measurement techniques that are needed. Notable examples of such organizations in the United States are the Occupational Safety and Health Administration, the National Institute for Occupational Safety and Health, the Bureau of Radiological Health, and the Consumer Product Safety Commission. An example of such an institution on an international scale is the World Health Organization, which has recently established a program for standardization of protection against non-ionizing radiation (which includes the ultraviolet). In fact, the U.S. Bureau of Radiological Health (Dept. of Health, Education and Welfare) has been designated the primary collaborating center whose purpose is to assist the World Health Organization in:

(1) estimating present levels and trends of human exposure to non-ionizing radiation;

(2) promoting the establishment of instrument calibration and reference services in various countries;

(3) establishing internationally acceptable nomenclature, definition of terms and quantities, and dosimetry methods;

(4) training public health personnel;

(5) alerting health officials to the need for establishing non-ionizing radiation control programs; and

(6) stimulating international research on biological effects and development of measuring instrumentation.

Little information has been found concerning state, local, and voluntary participation in ultraviolet standards specification. This is not because there are not enough users or no causes for concern--in fact, there are presently several million germicidal lamps in the country, including a set of such lamps in every hospital operating room. It is also not because ultraviolet radiation products have only recently become available in the market place--in fact, many American school rooms in the early 50 's were equipped with germicidal lamps to prevent the spread of bacteria.*

*I personally recall seeing them for the first time in 1949 when polio was a serious problem in my elementary school. 
Rather, there are few local standards committees because the technology for producing and measuring the radiation has been extremely slow in developing, thus making specifications of any kind difficult to decide upon. It is only in the last eight years that the national regulatory agencies mentioned above have been organized, and they are still struggling with the measurement problem (see section 2.3.4).

Because there has not been a clear appreciation for the need of an interacting national measurement system, there has been little communication between the academic community, the instrumentation industry, the users groups, and the national laboratories. It was as if each application took place in a technological vacuum. This situation can be illustrated, in a general way, by the following likely sequence of events. Looking back at the last 30 years, it is clear that ultraviolet lamps existed and were used. However, without standards, reports of the users were sometimes incomplete, misleading, or at least ambiguous. Without good diagnostics based upon reliable standards the application of the technology to other problems areas was severely hampered; results could be published but the science which could adequately explain the results and enable generalizations, extrapolations, and improvements was missing. Without economic stimulation from a diverse base of customers, the instrumentation industry did not expand or improve on its products. Without this type of activity, the standards laboratories assumed that their assistance or guidance was not required. Without the standards from the standards laboratories there was little uniformity, and the regulating bodies found that it was impossible to draw up realistic specifications. Without the documentary specifications and standards, the user was left with applications or experiments which could only be described in a qualitative way. And so the vicious circle continues.

\subsubsection{Survey of Documentary Standards}

As one might anticipate from the discussion in the previous section, documentary standards for far ultraviolet radiation are not widespread. At the present time there are none at all for radiation below $200 \mathrm{~nm}$. For an idea of the variety and significance of documentary standards which apply for the most part to visible radiation but which also touch lightly on the near ultraviolet and may eventually extend into the far ultraviolet, the reader is referred to the microstudy on Radiometry and Photometry. A few examples are briefly discussed here.

The American National Standards Institute (ANSI), a group which is set up to coordinate the development of national consensus standards established by other voluntary standards organizations, has recommended procedural standards to protect arc welders from face and eye injuries due to ultraviolet radiation produced in welding operations. Goggle transmittance (ANSI

287.1-1968-6.3.4.6), the proximity of coworkers, wall reflectivity, and shielding materials (ANSI Z49.1-1973-7.2.3) are typical areas of concern.

The National Institute for Occupational Safety and Health has recommended to the Occupational Safety and Health Administration standards of a more technical nature in its publication Criteria for a Recommended Standard ... Occupational Exposure to UTtraviolet Radiation [1]. Included in this document was input received from the American Conference of Government Industrial Hygienists, a voluntary group active in developing and recommending standard procedures affecting radiation safety. Indicative of the improving technology, this document defines the safety problems in terms of the absolute intensities and spectral irradiance levels involved rather than such relative quantities as, for example, goggle transmittance, distance from "typical" lamps, etc.

Whereas the National Institute for Occupational Safety and Health is concerned with the individuals who are being irradiated by ultraviolet light and the irradiance level at a particular location on a work site, the Bureau of Radiological Health is charged with regulating the sources of radiation themselves and is currently working towards establishing suitable standards and controls. They address themselves to such questions as the following. Do potentially dangerous ultraviolet lamps carry appropriate labels or warnings? Should certain lamps be required to have shielding? Under what conditions are ultraviolet lamps dangerous? The first ultraviolet product standard to be issued by the Bureau of Radiological Health will deal with commercial sunlamps and it is expected that significant documentary standards will be forthcoming from this regulatory agency.

Although our survey of documentary standards is brief and perhaps incomplete, it is apparent that many of those that address the wavelength range between 200-400 nm are often inadequate because of a confusion 
between radiometric units and photometric units.* A major manufacturer of laboratory weathering and colorfastness test equipment describes this situation as follows:

"These instruments enjoy international use and are specified in commercial and government standards for evaluating the durability of a wide variety of materials.... The International Standards Organization, Technical Commitee 61 on Plastics, is presently considering standards of light intensity. Confusion exists through the present use of luminous standards, e.g., lux, rather than radiometric standards."

\subsubsection{Instrumentation System}

\subsubsection{Measurement Tools and Techniques}

Various technical reviews on ul traviolet instrumentation are available in the open literature. For example, one whole issue of Electro-Optical Systems Design [3] has been devoted to this subject and includes authoritative discussions of: (1) ultraviolet lasers; (2) optics in the vacuum ultraviolet; (3) far ultraviolet filters; (4) reflective coatings; (5) diffraction grating; (6) polarizers; and (7) far ultraviolet detectors and sources. More specialized reviews on ultraviolet sources and detectors are available, for example, a review [4] which is devoted to applications in the photochemical industry. Likewise, there have been many specialized reviews on the status and applications of ultraviolet lasers (see for example $[5,6,7]$ ).

Rather than repeat much of what has already been described in the literature, we have decided to concentrate on reviewing only the type of primary and secondary standards which have been used in the various ultraviolet radiometric applications. These are illustrated on a wavelength scale in figure 2 (standard sources) and figure 4 (standard detectors). The scales are logarithmic and extend from $1 \mathrm{~nm}$ in the extreme ultraviolet to $1000 \mathrm{~nm}$ in the infrared. ${ }^{\dagger}$ The figures are meant to be representative and are essentially complete only for the ultraviolet region below $300 \mathrm{~nm}$. Primary standards and their regions of application are listed above the scale; secondary or

*Photometric units involve the standard response of the eye and can only be applied, by definition, to visible light [2].

†Figures 2, 4, and 8, have identical scales in order to facilitate comparison of the information content in each figure.

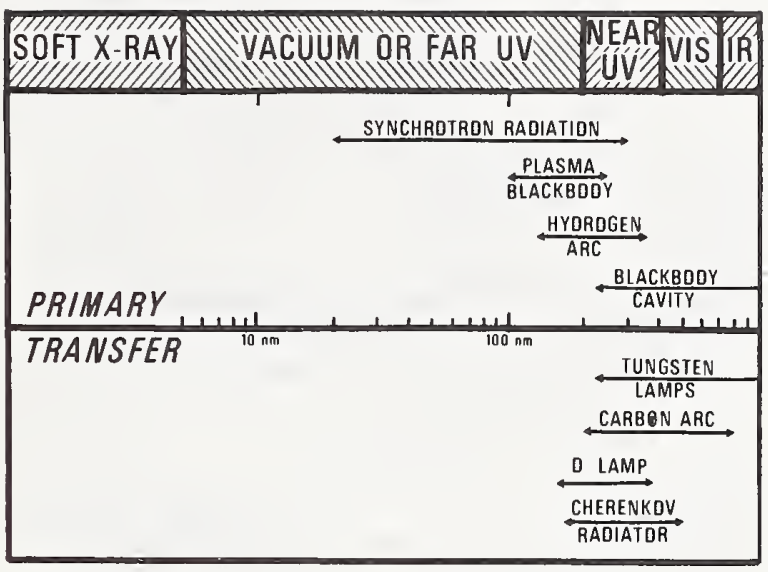

Figure 2. Primary and transfer standard sources which have been applied in the ultraviolet.

transfer standards are listed below the scale. A short description of each of the listings is contained in the following. Where it seems appropriate, relative advantages and disadvantages of a particular technique will be indicated.

\subsection{Standard Sources}

The primary and transfer source standards included in figure 2 are discussed in the following. For comparison purposes the spectra of several of the sources are shown in figure 3.

(1) Conventional Blackbodies. These are hollow cavity sources designed to closely approximate the ideal blackbody, which may be defined as a surface that absorbs all incident radiation and reflects none. This ideal is approximated by an enclosure whose walls and contents are all at a common temperature (isothermal enclosure) and impervious to radiation. The radiation leaving the walls consists almost entirely of radiation emitted by them. The spectral radiance of the radiation leaving a small hole in the enclosure is given closely by Planck's law and is a function only of the temperature of the enclosure. The temperature is related to the international practical temperature scale (IPTS) by operating the source at the melting point of a noble metal such as gold [8].

Strengths: Technique is accepted and used on a worldwide basis. Very high accuracy is possible. 


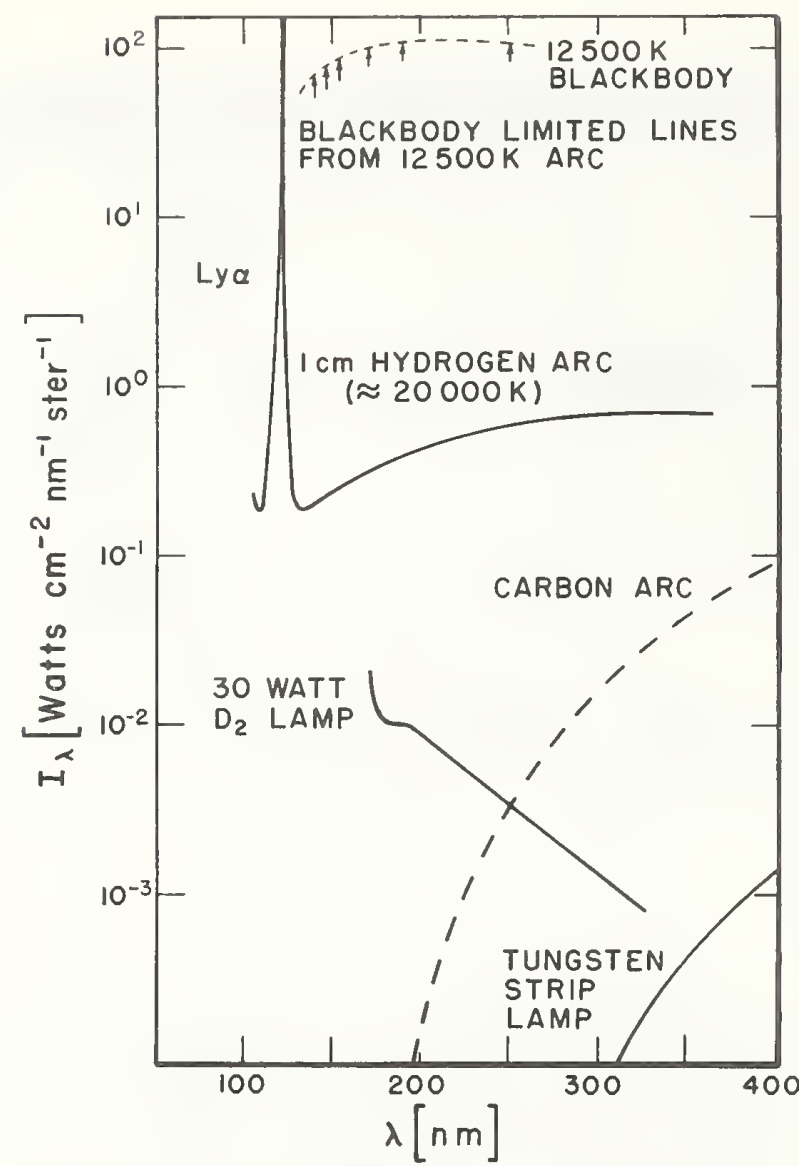

Figure 3. Comparison of the spectral radiance of several ultraviolet standard sources at typical operating conditions.

Limitations: The ultraviolet continuum at the gold point is extremely weak. Applications are limited to wavelengths greater than about $250 \mathrm{~nm}$.

(2) Plasma Blackbodies. These are high temperature noble gas stabilized arc discharges which are used to produce plasmas whose radiation absorption coefficient is equal to or greater than $5 \mathrm{~cm}^{-1}$ (optically thick plasmas). This is accomplished either by using very high pressures to increase the continuum absorption coefficient [9] or by operating only at wavelengths where atomic transitions with very high absorption coefficients (resonance lines) exist [10]. Strengths: Technique is applicable in principle down to $50 \mathrm{~nm}$.

Limitations: Relatively high uncertainties (10 to 25 percent) due either to uncertain temperature determination or lack of plasma homogeneity.

(3) Hydrogen Arc. This is a stabilized arc plasma which emits optically thin continuum radiation between $360 \mathrm{~nm}$ and $130 \mathrm{~nm}$ [11]. Because the continuum absorption coefficients are exactly known for atomic

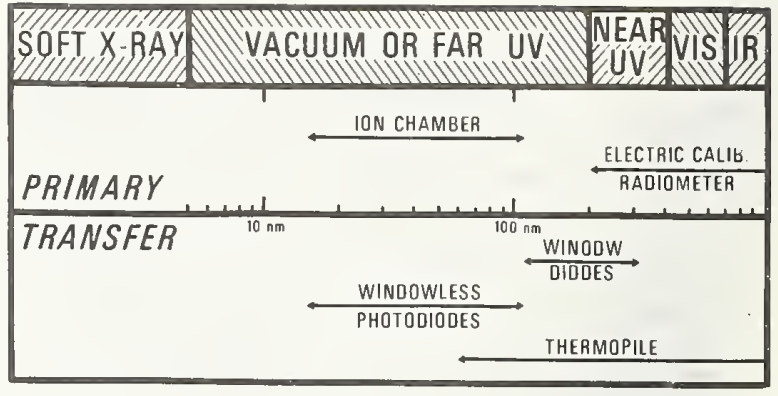

Figure 4. Primary and transfer standard detectors which have been applied in the ultraviolet.

hydrogen and because the conditions for 10cal thermodynamic equilibrium can be produced in the arc, the emission coefficient can be calculated as a function of temperature. However, extensive temperature diagnostics are not necessary and do not limit the ultimate accuracy since the arc can be applied as an absolute standard of spectral radiance by increasing the arc temperature to such a value that the spectral radiance reaches a unique and calculable maximum where the two major competing processes, increasing ionization and the lowering of total number density, provide an optimum balance.

Strengths: High intensity continuous spectrum ranges from visible into the vacuum ultraviolet with relatively low uncertainties (5 percent).

Limitations: High power $(100 \mathrm{~kW})$ is required; $\mathrm{H}_{2}$ molecular absorption lines limit use below $130 \mathrm{~nm}$.

(4) Synchrotron Radiation. The theory of synchrotron radiation from accelerated electrons is on excellent theoretical grounds, and the continuum emission can be quantitatively predicted when the energy of the electrons, the radius of the circulating orbit, and the electron current are known. The first two quantities can be determined typically to better than 0.5 percent, and the electron current is determined either by direct counting or by radiometry in the visible region where relatively high accuracy can be achieved $[12,13]$.

Strengths: An intense source of continuum radiation which covers the entire ultraviolet region. Polarization characteristics are desirable for certain applications. Iimitations: Major facility, not portable, high vacuum requirement; highly polarized radiation is undesirable for certain applications.

(5) Tungsten Lamps. Tungsten strip 1 amps are used as transfer standards of 
spectral radiance, and tungsten quartzhalogen lamps are used as transfer standards of spectral irradiance [8]. The tungsten resistive element is heated to incandescence by a dc electric current. The continuum spectral output is similar to that of a $2600 \mathrm{~K}$ blackbody.

Strengths: Techniques are well understood and uncertainties on the order of 1 to 3 percent are possible.

Iimitations: Weak source in the ultraviolet. Much more intense visible light spectrum can cause serious scattered light and out-of-band rejection problems when making measurements below $300 \mathrm{~nm}$.

(6) Deuterium Arc Lamps. These have been applied as transfer standards of both spectral radiance $[14,15]$ and irradiance [16]. A short L-shaped arc discharge is formed between to electrodes set at $90^{\circ}$ and excites a molecular $D_{2}$ continuum extending from $167 \mathrm{~nm}$ to about $400 \mathrm{~nm}$.

Strengths: Very strong ultraviolet output, portable, low power requirements.

Limitations: Alignment is difficult, and there are uncertain long-term aging effects.

(7) Carbon Arc. This is a transfer standard of spectral radiance [17]. A low current arc is formed between two electrodes so that the carbon anode is heated and emits a spectrum similar to that of a $3800 \mathrm{~K}$ blackbody radiator.

Strengths: Stronger in the ultraviolet than the tungsten lamps (but weaker than the deuterium lamps).

Limitations: Emission and/or absorption by the plasma cause relatively large uncertainties and limit the wavelength regions of applicability.

(8) Cherenkov Radiators. Radiation is produced when energetic beta particles emitted from a radioactive source pass through a medium having a high index of refraction such as quartz or magnesium fluoride. The beta particles travel at a speed greater than the speed of light in the refractive medium and emit characteristic Cherenkov continuum radiation [18]. The spectral distribution is given by theory. Absolute values are determined through normalization by comparison with a standard lamp in the visible.

Strengths: Requires no power supply and is portable.

Limitations: Possible radiation damage to the optical grade crystal; requires shielding to protect personnel and equipment from exposure to radioactive source. Radiant power output is very weak.

\subsection{Standard Detectors.}

The primary and transfer detector standards included in figure 4 are discussed are discussed in the following.

(1) Electrically Calibrated Pyroelectric Detector. This is a radiometer [19] which is based upon a pyroelectric detector and waveform-independent synchronous amplification. The receiver-heater is alternately exposed to the radiation field and to a calibrating electric current. A phase demodulator is used to provide for null measurements. Strengths: High accuracies and fast response times are possible.

Limitations: Cannot be used at wavelengths less than about $160 \mathrm{~nm}$ due to production of secondary electrons at the receiver.

(2) Ion Chamber. This is a gas filled detector in which each photon absorbed produces one electron-ion pair which is collected by a simple arrangement of parallel plates used to set up the collecting field. Absolute detection based upon a single chamber requires knowledge of the gas absorption coefficient and pressure. However, the more common use of a double ionization chamber [20] as an absolute detector does not require such diagnostics and depends only on an accurate measurement of the currents to the two identical chambers.

Strengths: High accuracy, easy to operate. Iimitations: Operates only for wavelengths short enough to exceed the photoionization threshold, but not so short that multiple ionization is possible.

(3) Window Photodiodes. This transfer standard is basically a vacuum photodiode in which ideally the only parameter to be determined by reference to an absolute standard is the absolute photoelectric yield of the photocathode as a function of wavelength [21]. The detectors are evacuated and sealed by an ultraviolet transmitting window to avoid atmospheric contamination of the surfaces. The uniformity of the quantum efficiency of the cathode can be excellent, on the order of 1 percent. Aging effects are likewise negligible.

(4) Windowless Photodiodes. Basically this is the same type of detector as described above in (3), but the lack of a window and the consequent occasional exposure of the photocathode to laboratory conditions affects the overall reproducibility. Nevertheless, there is evidence that natural aging effects cause not more than a 20 percent change in quantum efficiency over a three year test period. 
Strengths of (3) and (4): Wide wavelength region of applicability, relative high accuracy and stability.

Limitations of (3) and (4): These are low current devices (no amplification). If they are used to calibrate more sensitive photomultipliers, linearity and scaling problems with the latter introduce additional uncertainties.

(5) Thermopile. This transfer standard is based upon the voltage developed across an arrangement of thermocouple junctions heated by absorption of the incident energetic radiation [21]. The efficiency of the device is calibrated either by a spectral irradiance source in the near ultraviolet or by an ionization chamber in the far ultraviolet. The thermopile is designed so that the percent of radiation absorbed is independent of wavelength, and the detector is said to be uniformly grey. Thus, a thermopile can be applied in regions far removed from the calibration wavelengths.

Strengths: Wide wavelength range of applicability.

Limitations: Low sensitivity, not suitable for pulsed operation.

\subsubsection{The Instrumentation Industry}

The basic instrumentation involved in making measurements of ultraviolet radiation consists of detectors, analyzers (spectrometers, filters, and associated optical systems), and the radiation sources themselves. Consequently, the lamp industry, the optics industry, and the electronics industry all have an interest in ultraviolet measurement technology. This section describes the nature and extent of that interest. In particular, it shows that: (I) the instmumentation industry is seriously involved in the business of ultraviolet radiation measurements;

(2) there are sufficient customers (or users) to make a significant economic impact both on a national and an international scale (see also section 3.1.2.(3)); and (3) the traceability of the products to reference standards is tenuous at best and in many cases it is entirely inadequate.

A partial listing of ultraviolet lamp manufacturers and distributors is shown in appendix B. This list is reproduced from a 1973 issue of the trade journal, ElectroOptical Systems Design.* It is probably not

\footnotetext{
*A few other categories besides ultraviolet radiation products are also scattered throughout the list. The journal publishes such a list annually and can provide more recent compilations to interested readers.
}

Table 1. The value of shipments of a variety of ultraviolet emitting products for three different years according to the U.S. Census of Manufactures.

\begin{tabular}{|c|c|c|c|c|}
\hline \multirow{2}{*}{$\begin{array}{l}\text { SIC Code } \\
(1970)\end{array}$} & \multirow[b]{2}{*}{ Description } & \multicolumn{3}{|c|}{ Value of Shipment } \\
\hline & & 1967 & 1963 & 1958 \\
\hline 36410.41 & Germicidal lamps & 1.1 & 1.1 & 1.0 \\
\hline 36410.42 & $\begin{array}{l}\text { Sunlamps } \\
\text { Sump }\end{array}$ & 3.2 & 1.7 & 2.4 \\
\hline 36410.51 & Glow discharges & 7.4 & 5.8 & 2.8 \\
\hline 36410.52 & High intensity discharges & 27.6 & 17.3 & $8.8-1-1-1)$ \\
\hline 36410.53 & Photochemical discharges & 3.6 & 1.7 & 2.0 \\
\hline 36423.14 & Industrial mercury fixtures & 17.7 & 2.1 & 10.0 \\
\hline 36425.13 & Outdoor mercury lights & 18.3 & 11.9 & 5.1 \\
\hline 36425.14 & Outdoor mercury lights & 35.5 & 23.6 & 5.1 \\
\hline 36425.19 & Mercury floodlights & 20.2 & 8.0 & - \\
\hline 36425.25 & Mercury decorative lights & 9.0 & 1.3 & - \\
\hline & Fluorescent 11ghts & 9.0 & 4.5 & - \\
\hline \multirow[t]{2}{*}{36426.21} & MIscellaneous discharges & 6.7 & 5.3 & 3.6 \\
\hline & Totals & 140.3 & 84.3 & 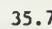 \\
\hline
\end{tabular}

complete, as it emphasizes primarily small businesses. The giants of the trade, Sylvania, General Electric, ITT, Westinghouse, etc., must be added to such a listing and are responsible for much of the research and development on the lamps.

An idea of the impact of these industries can be obtained in the U.S. Census of Manufacturers. For example, the section on electric lamps (SIC Code 3641) and lighting fixtures (SIC Code 3642) includes the data listed in table 1.*

According to one industry official, more than 100 million mercury vapor lamps have been sold for outdoor illumination purposes since their introduction 40 years ago. The reason for their popularity is primarily because electric discharge lamps of this type are very efficient: they typically produce almost four times more illumination than an incandescent light bulb using the same amount of electricity. Of course, in this application the lamp industry is most interested in minimizing the ultraviolet output (a measurement need) and thus maximize the overall illumination efficiency.

\footnotetext{
*The 1972 Standard Industrial Classification changed the code for commercial lighting fixtures to 3646 . The code for electric lamps was unchanged. However, the 1970 codes were preferred for illustration in table 1 since they make it possible to specify the subset which includes only ultraviolet emitting lamps.
} 
On the other hand, there are other illumination applications, for example those involving indoor lighting and solar simulators, where, according to an industry spokesman:

"... it is frequently essential to compare the ambient ultraviolet output of a

lighting installation with that of natural daylight conditions."

The significance of the measurement problem is pointed out as the statement continues:

"... the accurate uv measurements of energy from light sources and natural daylight have been hampered by the lack of reference standards."

There is an increasing variety and sophistication of products available from the lamp and electro-optics industry: over 350 fluorescent lamps including 19 colors, metallic and mercury lamps, light emitting diodes (LED), and new radiometers and spectrophotometers. The U.S. market for spectrophotometers alone is about $\$ 100$ million per year. However, it is estimated that about 40 percent of this is imported compared to an export market of only about 10 percent [22]. Two major lamp manufacturers have considered this trade deficit:

"Expansion of lamp business offshore is hindered by the lack of a uniform measurement level of luminous flux..

"There will certainly be an unfavorable foreign trade impact if electro-optical devices of U.S. manufacture cannot be relied upon to yield uniform NBS traceability quantities."

One of the principal manufacturers of near and vacuum ultraviolet spectrometers is in the U.S. and has sold now more than 300 instruments, each representing a value of about $\$ 20,000$. About 70 of the instruments have been bought by major U.S. technological companies, which is some indication of the attention that ultraviolet radiation receives in industrial research. However, it appears that Japan and France are preparing to market some new low cost ultraviolet spectrometers, which utilize recently developed holographic gratings. In our opinion these may seriously affect the sales of the U.S. companies. Quality control is then the key and a hint at what U.S. manufactures may do to secure their business is contained in a letter from the president of one of these companies:

"...During the past year there have been several requests from our customers for information on the availability of light sources calibrated for outputs below 300 nm... Such a source would be of value in calibrating the absolute transmission of our monochromators, a question we are constantly asked, A standard light source and wide range detector would probably be of considerable value to our customers for calibrating each specific system they may assemble."

It may be implied from this letter that the market for ultraviolet spectrometers and instrumentation would be more secure and perhaps larger if standard data and ultraviolet techniques were readily available. Certainly if the U.S. instrumentation industry is to continue to be competitive in the world market, the following situation, described to us by an industrial scientist, must be improved:

"... We have had the experience of purchasing a uv meter which, when compared to lab standards, had an error in excess of $100 \%$."

Ultraviolet emitting lamps are being used increasingly in the chemical and drug industries and in biomedical research. Applications include fluorescence analysis, detection of ultraviolet absorbing chemicals. quality control in the production of pharmaceuticals (testing for fluorescent contaminants), germicidal lamp installations (for air and food sterilization), photo-polymerization, and mutagenic studies. For the characterization of the emission of these lamps, calibration is absolutely essential. The following statements are examples of the interest two major lamp and detector manufacturers have taken in the development of radiometric standards in the ultraviolet.

"The development of the quartz halogen

incandescent standards of spectral irradiance was of great significance, and the availability of a similar standard in the ultraviolet is greatly to be desired. This need has been with us for a number of years and is growing steadizy more urgent. We will be looking forward with great interest to the results of your proposed program.

"We urgently need a standard uv source to provide us with an absolute calibration. We supply, with each of our detectors, an individually measured absolute spectral response calibration.... Clearly, we require absolute calibration information to maintain the accuracy of this service. Presently, we cannot maintain our absolute uv calibration accuracy to the accuracy which we desire.... We would like to cooperate with NBS in any way 
possible on this problem."

\subsubsection{Reference Data}

Quantitative spectroscopy* is a preferred diagnostic method essentially because it is a non-perturbing technique. Also it can be applied to remote sources (as in the case of the sun). Ultraviolet radiation measurements which are made through the use of the lamps, analyzers, and detectors previously discussed are often used for this purpose. For example, plasma physicists study the ul traviolet radiation from fusion devices in order to determine the identity and density of contaminants which absorb energy, radiate at characteristic wavelengths, and thus cool the plasma before fusion conditions can be achieved. In the same way, space scientists, astrophysicists, and aeronomers study solar, stellar, and atmospheric radiation in order to determine particle densities which are necessary for modeling of the system under investigation. In order to make such connections, certain basic quantities must be known. One example is the transition probability, $A_{k}$, defined as the probability that an atom or molecule in a particular excited state $k$ will decay with the emission of a photon to a lower excited state $i$. The emission coefficient of the radiation is given by the product of the transition probability, the number density of excited states $N_{k}$, and the photon energy $h c^{-1}$,

$$
\varepsilon\left(W m^{-3} s r^{-1}\right)=\frac{1}{4 \pi} \frac{h c}{\lambda_{k i}} N_{k} A_{k i} \text {. }
$$

Universities, government laboratories, and several industrial research groups around the world are involved in determining such basic atomic constants. The methods of acquisition, the sources of the data, and a discussion of uncertainties are discussed for example in recently available bibliographies and data compilations $[23,24]$. Experimentally, the determination of $A_{k i}$ very often requires the absolute measurement of intensity. For example, a laboratory emission experiment based on equation (2) can determine $A_{k i}$ through an absolute intensity measurement $(\varepsilon)$ and one other diagnostic measurement to determine $\mathrm{N}_{\mathrm{k}}$.

Equation (2) has also been applied in such a way that ultraviolet calibrations can be made without the direct use of any ultraviolet intensity standards. This is the so-called branching ratio method used often

\footnotetext{
* In general, this can be defined as the measurement of the wavelengths and intensities of radiant energy.
}

by fusion scientists [25] to determine absolute intensities in wavelength regions where there are no ultraviolet standards at all. If a particular excited level $\mathrm{N}_{k}$ decays to several lower levels $i$ and $j$ with the emission of photons with wavelengths $\lambda_{k j}$ and $\lambda_{k j}$, the ratio of the emission coefficients is given by

$$
\frac{\varepsilon_{k i}}{\varepsilon_{k j}}=\frac{\lambda_{k j}}{\lambda_{k i}} \frac{A_{k i}}{A_{k j}}
$$

If the branching ratio, i.e., the ratio of the transition probabilities, is known and if $\lambda_{k j}$ is in the visible so that $\varepsilon_{k j}$ can be measured by conventional radiometry, $\varepsilon_{k j}$ is determined. This method has the advantage that the diagnostic (which is often quite complicated and model dependent) for $N_{k}$ is unnecessary. It has the disadvantage that nature is perverse enough that it provides fewer and fewer branching possibilities as the wavelength difference between the two emission lines increases. This situation obviously improves greatly if a modest ultraviolet radiometric capability is available so that the requirement that one of the lines be emitted in the visible is not so limiting.

There is a similar need in the National Measurement System for other basic data such as collision cross sections and reaction rates, and in the same way as above, absolute radiometry is often useful in determining these quantities in the laboratory. Space science, fusion research, upper atmosphere physics, laser research-all are impact areas in the ultraviolet radiometry measurement system and depend on the availability and accuracy of such data.

\subsubsection{Reference Materials}

Spectrophotometric standards of transmittance and reflectance are available in the visible region of the spectrum at NBS. In addition spectral transmittance standards whose calibration extends to wavelengths as low as $240 \mathrm{~nm}$ in the near ultraviolet can be obtained. The reference material is a holmium oxide square glass plate (Corning $3130 \mathrm{glass}) 2.5 \mathrm{~mm}$ thick. These standards are used for checking the transmission calibrations of recording spectrophotometers which are used primarily in absorption spectroscopy and spectrochemical analysis.

As previously mentioned, the economic impact of the spectrophotometry industry is estimated to be about $\$ 100$ million per year. Typical uses of calibrated spectrophotometers are: 
(1) clinical examination (analysis of $\mathrm{Ca}$ and $\mathrm{Mg}$ in brood and urine);

(2) metal industry (analysis of $\mathrm{Mg}, \mathrm{Ni}$, $\mathrm{Mn}$, and $\mathrm{Pb}$ in iron and stee 1 );

(3) agricultural industry (analys is of $\mathrm{Cu}$ and $Z n$ in fertilizers); and

(4) chemical industry (analysis of $\mathrm{Mg}$ in cement, analysis of $\mathrm{Pb}$ in petroleum products).

Most of these instruments can be operated down to $195 \mathrm{~nm}$ (the cutoff of air). In the opinion of a senior product manager for a spectrophotometer manufacturer,

"The most widely used method in medical analysis (of biological fluids) has been ultraviolet spectroscopy."

It is evident that the wavelength range of standard reference materials presently available is not sufficient to meet current demands. *

Vacuum ultraviolet spectrophotometers are not yet commercially available, but one may anticipate their application in the near future. Because absorbance in the vacuum ultraviolet is extremely strong due to atomic and molecular resonance transitions in this region, the sensitivity and range of the spectrophotometric techniques could be greatly improved. Also new techniques could be developed based on the fact that the energy thresholds for dissociation of complex molecules occur in this region. For example, identification and quantification of compounds by the measurement of abrupt steps in the continuous absorption background could be made. Calibration of these instruments with standard filters will then be a necessity. Although no coordinated standard reference materials program for the wavelength region under $200 \mathrm{~nm}$ exists as yet, the technology to provide such materials is available. Several companies now provide calibrated transmission filters down to 110 $\mathrm{nm}$ and have provided reflectance calibrations down to $50 \mathrm{~nm}$.

\subsubsection{Science and People}

To us, the phrase "science and people" means communication. The ultraviolet radiation measurement system is supported by scientists involved in such diverse fields as atomic and molecular physics, plasma physics, physical chemistry, space science, astrophysics, aeronomy, meteorology, photobiology, and biomedical research. Direct.

*The Radiometric Physics Section (232.14) at NBS has a program working to extend the short wavelength 1 imit of spectral transmittance standards to $200 \mathrm{~nm}$. contact between such specialized groups of people is unfortunately infrequent and occurs usually through the mechanism of invited papers at conferences. Publication of review papers in journals dedicated to fields outside of one's own speciality is another obvious possibility for contact, but it is a very unusual occurrence mainly because writing for a group with different. backgrounds is not an easy task and sometimes not very rewarding from the viewpoint of feedback. Such information activities (conferences, workshops, and publications) usually come under the auspices of the relevant professional societies such as the American Physical Society (APS), the Optical Society of America (OSA), the Institute of Electrical and Electronics Engineers (IEEE), American Astronomical Society (AAS), American Geophysical Union (AGU), American Biophysical Society (ABS), and the American Society of Photobiology (ASP).

Perhaps the group who has the best overa11 picture of the measurement system is the instrumentation industry and in particular the local sales representative whose business it is to know the present and anticipate the future needs of the various measurement sectors. He also provides sometimes the only link between the bench scientist and the outside world which is involved in testing, development, production, and evaluation programs.

Perhaps the most influential role in the dissemination of knowledge is played by the trade journals, both directly through their own distribution system and indirectly through the popular press which monitors the journals for especially significant activities and trends. Examples of such publications reporting on ultraviolet radiation developments (among other things) are Industrial Research, Electro-Optical Systems Design, Optical Spectra, Laser Focus, and Measurement and Data News, a 11 of which are freely distributed and are supported entirely by instrumentation advertisements. Some of these organizations are quite innovative and manage very effectively in stimulating interest in science and its applications. For example, Industrial Research sponsors a wel1-respected annual IR-100 award program for the 100 most significant developments in any and all fields of science.

\subsection{Realized Measurement Capabilities}

In section 3 the many areas of impact for an ul traviolet measurement capability are described in detail. The wavelength range of interest spans from $4 \mathrm{~nm}$ to $300 \mathrm{~nm}$ 
and includes a wide variety of phenomena and applications essentially because the corresponding photon energy extends from $300 \mathrm{eV}$ to $4 \mathrm{eV}$. Consequently, it is very difficult to unequivocally specify the accuracies required in the far ultraviolet. In fact, one risks overspecifying the accuracies if he insists on a simple formula. The accuracy requirements depend not only on the wavelength region but also on the manner in which the measurements are used. For example, ultraviolet measurements that are being used to generate data which are, in turn, used for trend determinations or scaling predictions require much greater accuracies than ordinary laboratory or industrial applications.
(CORM) ${ }^{+}$has estimated that at least 5 percent absolute accuracy is required by indus try and the scientific community. Below $90 \mathrm{~nm}$ we enter into the region where fusion scientists are most interested and where scaling is extremely important. It is estimated that 10 percent uncertainties would be acceptable for now in this very difficult spectral region. Because very expensive machines are being designed according to these scaling estimates and because the stakes are so high (energy crisis), an active radiometry effort below $90 \mathrm{~nm}$ is considered to be a high leverage program.

The above accuracies would certainly satisfy most of the sectors described in this survey. Most of them would probably

Table 2. An overview of the far ultraviolet radiometric needs and capabilities of the National Measurement System.

\begin{tabular}{|c|c|c|c|c|c|}
\hline \multirow{4}{*}{$\begin{array}{l}\text { Wavelength } \\
\text { Region ( } \mathrm{nm})\end{array}$} & \multirow{4}{*}{$\begin{array}{c}\text { Uncertainties } \\
\text { Required }\end{array}$} & \multicolumn{4}{|c|}{ Uncertainties in the Standards } \\
\hline & & & \multirow{2}{*}{$\begin{array}{l}\text { Detectors } \\
\text { Irradiance }\end{array}$} \\
\hline & & \multicolumn{2}{|c|}{\begin{tabular}{|c|c|}
\multicolumn{2}{c|}{ Sources } \\
Spectral Radiance & Spectral \\
\end{tabular}} & \multirow{2}{*}{ Spectral } & \\
\hline & & \multicolumn{2}{|c|}{ Primary Transfer } & & Transfer \\
\hline $250-300$ & \multirow{6}{*}{$\begin{array}{r}1 \% \\
1 \% \\
1 \% \\
5 \% \\
10 \% \\
10 \%\end{array}$} & \multirow{6}{*}{$\begin{array}{c}1-5 \% \\
1-5 \% \\
5 \% \\
10 \% \\
10 \% \\
10 \% \star\end{array}$} & \multirow{6}{*}{$\begin{array}{c}1-10 \% \\
1-10 \% \\
10 \% \\
10 \% * \\
\text { None } \\
\text { None }\end{array}$} & \multirow{6}{*}{$\begin{array}{l}5 \% \\
10 \% * \\
\text { None } \\
\text { None } \\
\text { None } \\
\text { None }\end{array}$} & \multirow{6}{*}{$\begin{array}{r}6 \% \\
6 \% \\
6 \% \\
6-10 \% \\
10 \% \\
10 \%\end{array}$} \\
\hline $200-250$ & & & & & \\
\hline $160-200$ & & & & & \\
\hline $100-160$ & & & & & \\
\hline $50-100$ & & & & & \\
\hline $5-50$ & & & & & \\
\hline
\end{tabular}

* Estimated from work in progress at NBS

Table 2 provides an overview of the measurement needs and capabilities of the national measurement system organized roughly into six wavelength regions throughout the far ultraviolet spectrum. In the ultraviolet range, $200-300 \mathrm{~nm}$, spectral radiance and irradiance uncertainties on the order of 1 percent are now required. In this region, the uncertainties of NBS ultraviolet source and detector standards range from 5 to 10 percent. Spectra] radiance standards with uncertainties on the order of 1 percent are available above $230 \mathrm{~nm}$, but are primarily for use in the visible spectrum since they have very low output in the ultraviolet. Between $80 \mathrm{~nm}$ and $250 \mathrm{~nm}$, the Council on Optical Radiation Measurements be content with even less, especially in areas where there is currently little input from the standards community. In this respect, one should note the conspicuous

\footnotetext{
${ }^{\dagger}$ CORM is a group of mostly industrial scientists and engineers interested in radioetry and photometry who are organized as an activity of Technical Committee 1.2 on Photometry of the United States National Committee of the CIE (Commission Internationale de L'eclairage) for the purpose of identifying the most urgent problems and projected national needs in the area of optical radiation standards.
} 
absence in table 2 of activity towards the development of standard sources of spectral irradiance which happens to be the radiometric quantity of principal interest to most of the users. Also, al though calibrated detectors seem to be in relatively good shape, one should keep in mind that the determination of spectral irradiance requires a wavelength filter or monochromator to be used in conjunction with the calibrated detector, i.e. a spectroradiometer. Such a complication can double the uncertainties of a practical measurement if indeed a user has the necessary techniques at his disposal to calibrate the transmission of the filter or monochromator. In principle, the calibration of such spectroradiometers is possible. The provision of such a service is under consideration at NBS, but currently there is no activity, at NBS or any of the standards laboratories, in this area.

Some users find themselves in a position where they do not even know what accuracies to specify because they are caught in a circular dilemma. The Occupational Safety and Health Administration, for example, will have the authority to close down plants which do not conform to the maximum ultraviolet irradiance guidelines which are now under scrutiny. Therefore, they will also have a corresponding obligation to recommend an accurate dosimeter. However, the reference data on what radiation and how much is dangerous are sometimes extremely uncertain due to the limited description and knowledge of the sources and detectors used in the referenced experiments. In this case, uncertainty breeds even more uncertainty. If the regulatory agency satisfies itself with an equally uncertain dosimeter, the quality of the reference data is not going to improve, and the problem with specification of hazardous radiation remains the same. The regulating agency is forced either to compromise its standards or to be super-cautious and to overspecify by defining "safety" factors. The former option may affect our health and safety; the latter option may result in costly unnecessary modifications in industrial plants which may in fact be safe working places.

Ultraviolet radiation is a resource which is just beginning to be exploited on a significant scale. It is a difficult spectral region to work in but promises to be extremely rewarding, both socially and economically. The measurement accuracies recommended in this section are the minimum required if the full potential of this relatively large part of the electromagnetic spectrum is to be utilized. Recommendations of programs which would tend to fill in the gaps apparent in table 2 are contained in section 4.4 .

\subsection{Dissemination and Enforcement Network}

\subsubsection{Central Standards Authorities}

The problem of putting ultraviolet radiation intensity measurements on an absolute scale is of international interest. There. are active government standards programs in the following nations: (1) the United States, through NBS; (2) the United Kingdom, through the National Physical Laboratories (NPL) in Teddington, England; (3) France, through the Institut National de Metrologie (INM) in Paris; (4) West Germany, through the Physikalische Technische Bundesanstalt (PTB) in Braunschweig and Berlin; and (5) Canada, through the National Research Council in Ottawa and the Ministry of Municipal Affairs in Montreal. This section provides a brief description of the ultraviolet radiometric capabilities of some of the recognized national standards laboratories and discusses the relevant interactions between these bodies.

(1) United States. The National Bureau of Standards program on ul traviolet radiometry is carried out in four separate sections in the Optical Physics Division: Plasma Spectroscopy, Far Ultraviolet Physics, Optical Radiation, and Radiometric Physics. Far ultraviolet research is 1 imited to the first two sections whereas the emphasis in the third and fourth sections is on visible and near ultraviolet radiation. The general piogram consists of several complementary components and makes use of absolute detectors as well as standard radiation sources. A detailed description of current NBS activities and future plans is contained in section 4.2.

(2) United Kingdom. The National Physical Laboratory (NPL) in Teddington, England, has available a set of seven calibrated deuterium lamps (manufactured in England) as laboratory standards of ultraviolet spectral radiance in the wavelength range $200 \mathrm{~nm}$ to $400 \mathrm{~nm}$. The spectral distribution is determined using the Daresbury synchrotron as a standard source; the spectral radiance is put on an absolute scale by a direct radiance calibration with a tungsten strip lamp in the near ultraviolet range $350 \mathrm{~nm}$ to $400 \mathrm{~nm}$. The National Physical Laboratory services are of two types: (1) the customers' own sources can be calibrated directly, if of a suitable type; (2) deuterium discharge lamps can be supplied with absolute radiance levels as required.

Future plans are to extend the radiance calibration service to include the 
wavelength region $160 \mathrm{~nm}$ to $200 \mathrm{~nm}$ and to add a spectral irradiance calibration capability in the range $160 \mathrm{~nm}$ to $350 \mathrm{~nm}$. In this connection, members of the National Physical Laboratory staff have been in consultation with NBS staff concerning absolute calibrations in the vacuum ultraviolet (below $200 \mathrm{~nm}$ ) utilizing both plasma blackbody radiation and hydrogen arc radiation. The National Physical Laboratory is currently pursuing the former approach and has built an arc and power supply similar to those used both at NBS, at the PTB in Berlin, and at the Max-Planck Institut in Munich. In addition, NBS has supplied three standard photodiode detectors calibrated in the range $116 \mathrm{~nm}$ to $254 \mathrm{~nm}$ to help establish the National Physical Laboratory's irradiance capability. Interlaboratory comparisons of both source and detector standards are either anticipated or already in the planning stages.

(3) France. The Institut National de Metrologie (INM) in Paris, France, is currentiy developing a primary standard of spectral radiance in the ultraviolet region $110 \mathrm{~nm}$ to $400 \mathrm{~nm}$ utilizing the hydrogen arc approach first demonstrated at NBS. One of their chief investigators spent $1-1 / 2$ years at NBS as a visiting scientist engaged in far ultraviolet radiometry. Originally, the Institut National de Metrologie planned to employ a high pressure plasma blackbody radiator as a standard of radiance, but because of the large uncertainties involved [9], they have apparently abandoned this idea and are actively supporting a program similar to that of NBS.

A1though standard detector research, per se, is not being pursued, standard detectors are being used. Staff from the Centre National de la Recherche Scientifique (CNRS) in Verrieres-le-Buisson have obtained from NBS several photodiodes calibrated over the range $58 \mathrm{~nm}$ to $254 \mathrm{~nm}$. These have been used to calibrate a French collaborative experiment on the U.S. space satellite OSO I and for subsequent similar work.

(4) Germany. The West Germany far ultraviolet radiometry program is centered at the Physikalische Technische Bundesanstalt (PTB) in Berlin, and to some extent in Braunschweig. There are also important contributions from several other groups within the country. The Physikalische Technische Bundesanstalt contributed to establishing the carbon arc as a standard of radiance in the near ultraviolet [26]. More recent West German activities have been to utilize the DESY synchrotron in Hamburg as a primary standard of spectral radiance. Deuterium and mercury lamps have been calibrated with the DESY synchrotron in the wavelength region $165 \mathrm{~nm}$ to $300 \mathrm{~nm}$ [15] and in addition have been cross-calibrated with a carbon arc [27]. The group in Berlin has also crosscalibrated several of these lamps with blackbody-limited line radiation from an arc plasma [28]. Space scientists from the Naval Research Laboratory in Washington, D.C. have used similar transfer lamps calibrated against the original DESY calibrated lamps for calibrating their rocket and satellite solar ultraviolet spectrometers.

A group of scientists at the Max-Planck Institut für Physik und Astrophysik in Munich have pioneered blackbody-limited line radiation [10] as a standard source of radiance and have been using the wall-stabilized arc source to calibrate rocket spectrometers in the wavelength region $110 \mathrm{~nm}$ to $300 \mathrm{~nm}$ for the last few years. Another group located at the Technische Universität in Munich is also active in ultraviolet radiometry. One of the staff has spent a year at NBS and has cooperated in further developing the NBS hydrogen arc radiance standard.

In summary, there are presently three separate efforts, as yet not totaliy coordinated, toward providing a national ultraviolet radiometric capability in west Germany: (1) the synchrotron effort in Hamburg; (2) the blackbody line radiation efforts in Berl in and in Munich; and (3) the hydrogen arc effort in Munich.

NBS interaction with the West German program is very strong. NBS has furnished calibrated photodiodes which are being used to conduct a comparison with several synchrotron-calibrated deuterium lamps. Consistency appears to be at least within 20 percent $[29,30]$. NBS has also calibrated several descendants of the original DESYcalibrated deuterium lamps with the NBS hydrogen arc and compared results [31] with the synchrotron based calibration. Again, consistencies within 20 percent were found except in one region where it appears that a systematic error affected the synchrotron calibrations [29]. Plans are currently being made to exchange deuterium lamps in a round robin calibration comparison between NBS, the DESY synchrotron group, the Physikalische Technische Bundesanstalt Berlin group, and the Institut National de Metrologie Paris group.

Independent of these events, the arc group at the Munich Max-Planck Institut has requested and received several NBS calibrated ultraviolet detectors. They have recently developed a facility whereby they can utilize their standard arc source to calibrate detectors such as photodiodes and photomultipliers. Preliminary results of a comparison of detectors calibrated at several European laboratories (unpublished) 
show large discrepancies (up to a factor 2). It is hoped that the NBS calibrated photodiodes will help to clear up the present uncertain detector-calibration base-line in Europe.

(5) Canada. The interaction with the Canadian national laboratories has not been strong and so far consists only in an exchange of program information. In December 1972, NBS received a letter from the Radioprotection Section of the Ministry of Municipal Affairs, Montreal, requesting information on ultraviolet laboratory standard sources and accessories, material characteristics, and a bibliography on ultraviolet radiation. Their general program goals were also described:

"We are developing a program of protection against nonionizing radiation.... and are most concerned with the measurements of uv light intensities in absolute units using a spectroradiometer. It is of prime importance for us to have an ultraviolet lab standard source to check periodically the accuracy of that calibration."

Details of NBS activities in developing primary and transfer standards of spectral radiance were forwarded but no further interaction has taken place as yet.

(6) Others. Australia, Japan, East Germany, and the U.S.S.R. have programs in ultraviolet radiometry, but little information on these, besides several key literature references (see for example [32] and [33]), has been obtained as yet.

\subsubsection{State and Local Offices of Weights and Measures}

No information has been found. It is assumed that activity in this area of the measurement system will not become significant until the national regulatory agencies have successfully organized their own programs in ultraviolet radiation measurement and control.

\subsubsection{Standards and Testing Laboratories and Services}

There are several laboratories in the United States other than NBS which maintain a far ultraviolet detector calibration facility, for example Stanford University [30]. NBS has been in close contact with the group from Stanford through the years, and in fact, the head of the group has served on the NBS Optical Physics Division Review Panel. NBS has also been in contact with representatives of the detector industry which maintain standards laboratories. Out of these interactions have come two of the three ultraviolet detector types now available as calibrated transfer standards from NBS. Consideration of such properties as uniform response across the photocathode and aging has resulted in products which minimize degradation of the calibration upon transfer from NBS. The manufacturer of one of the standard photodiode types has demonstrated its continuing interest in the far ultraviolet standards field by conducting a round robin intercomparison of detector calibrations from various laboratories worldwide [35].

The situation with ultraviolet source calibration facilities in the United States outside of NBS is not nearly so well developed. Although there exist at least 21 independent commercial standards laboratories involved in radiometric and photometric measurements [22], most of these apparently are not aware of recent NBS activities in far ultraviolet radiometry. This is surely a weak link in the measurement system as it presently stands. This extraordinary situation is highlighted even more perhaps by the recommendation of the Council on Optical Radiation Measurement (CORM) that industry and the scientific community at large require at least 5 percent absolute accuracy for radiation measurements between $90 \mathrm{~nm}$ and $250 \mathrm{~nm}$. Apparently, the interest is there, but the communication channels are not.

The existence of this critical missing link in the National Measurement System can be considered to be one of the more important findings in this study. Only now, at the time of this writing, is the situation beginning to change. For example, NBS has been cooperating with the commercial standards laboratories to make available deuterium lamp standards of spectral radiance and irradiance in the near and vacuum ultraviolet. A recent advertisement [36] describing this new service is reproduced in figure 5 .

\subsubsection{Regulatory Agencies}

(1) Occupational Safety and Health Administration. The 0ccupational Safety and Health Act of 1970, Public Law 91-596, is designed to assure safe and healthful working conditions for the nation's working men and women. It is implemented by two major agencies, the Department of Labor with its Occupational Safety and Health Administration (OSHA) and the Department of Health, Education and Welfare with its National Institute of Occupational Safety and Health (NIOSH). OSHA, as a regulatory agency has the responsibility:

"... to promulgate, modify, and improve mandatory occupational safety and health 


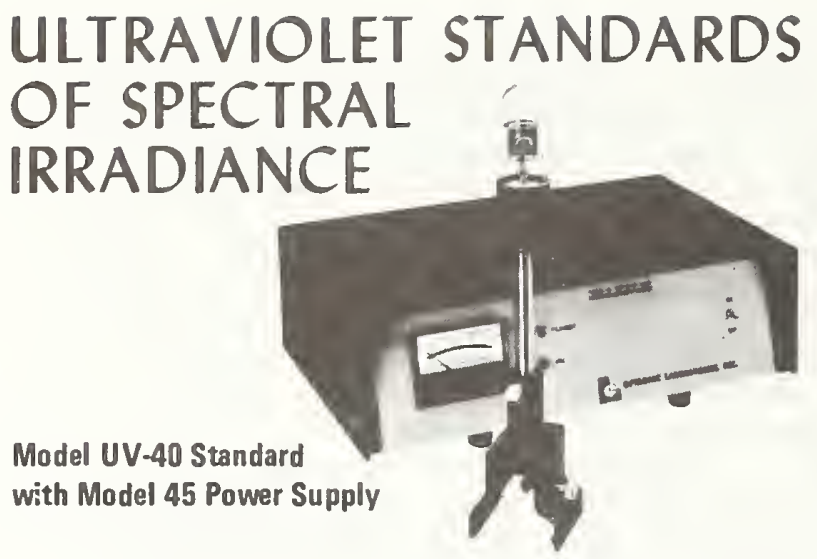

Optronic Laboratories is now issuing Deuterium lamp standards calibrated for spectral irradiance over the ultraviolet wavelength region of 1800 to $4000 \mathrm{~A}$. Calibration of the new standards is based on the NBS high-accuracy standards of spectral irradiance and on the NBS calibration of Deuterium lamps for spectral radiance. The standards typically exhibit a peak irradiance at $190 \mathrm{~nm}$ of $0.5 \mu W / \mathrm{cm}^{2} \mathrm{~nm}$. The lamps can also be obtained calibrated for spectral radiance.

Available for use with the new standards is the Model 45 Precision Constant Current DC Power Supply.

Figure 5. A copy of a publicity release illustrating a recent NBS cooperative effort with the instrumentation industry.

standards .... and to prescribe regulations requiring employers to maintain accurate records and reports concerning work-related injuries, illness, and death."

NIOSH, al though not a regulatory agency, has been given the responsibility, according to section 20(a)(3):

".... to develop and establish recommended occupational safety and health standards .....and to conduct such research and experimental programs necessary for the development of criteria dealing with toxic materials and harmful physical agents and substances which will describe exposure levels at which no employee will suffer impaired health or functional incapacities as a result of his work experience."

In order to provide relevant data from which valid criteria and effective standards can be deduced, the National Institute for Occupational Safety and Health has projected a formal system of research. As the research and epidemiologic studies are completed and sampling and analytic methods are developed, the criteria and standards will be reviewed periodically to ensure continuing protection of the workers. With the cooperation of an ad hoc committee of the American Industrial Hygiene Association,

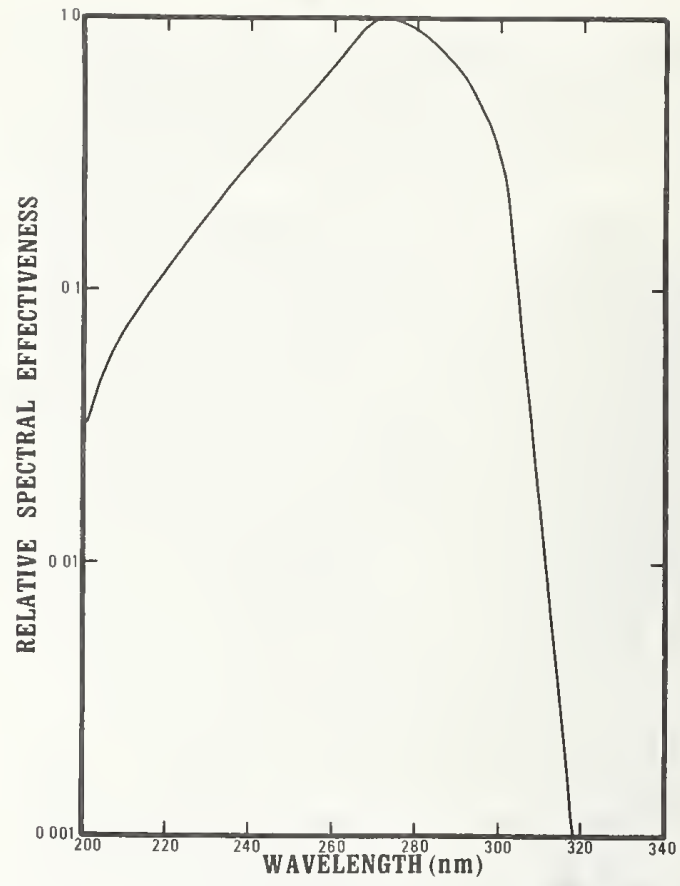

Figure 6. The ultraviolet radiation exposure standard recommended by the National Institute for Occupational Safety and Health is illustrated in terms of the relative spectral effectiveness in producing a standard erythema 1 response in the skin and eyes.

the American Medical Association Committee on Occupational Toxicology, and a number of review consultants, one such review has been published: Criteria for a Recommended Standard--0ccupational Exposure to UTtraviolet Radiation [1]. In addition, in view of their responsibility to determine the maximum allowable exposure levels for ultraviolet radiation, the Institute, in cooperation with the Department of Health, Education, and Welfare, the Department of Labor, and the Occupational Health and Safety Administration intends to develop a portable instrument for measuring these radiations so that both inspectors and industrial hygienists will be able to monitor the work environment. Two approaches are to be taken, both of which include NBS cooperation. First of all, they wish to develop a radiometer whose spectral response closely resembles the erythemal response of the skin and eyes. The desired spectral response is shown in figure 6 . This curve of relative effectiveness says, for example, that a given flux of radiation at $280 \mathrm{~nm}$ is about a factor 1000 more dangerous than the same flux at $200 \mathrm{~nm}$. The proposed radiometer or hazard meter would integrate over all wavelengths and record an effective irradiance which then can be associated with a maximum allowable exposure. Secondly, they wish to 
develop a standard source of irradiance which can be packaged along with the standard detector in order to enable the industrial hygienist to check periodically the calibration of his detector unit.

The interaction between the National Institute of Occupational Safety and Health and the standards community has been fairly strong. They have purchased a pair of deuterium lamps, which were calibrated at NBS in the wavelength region $200 \mathrm{~nm}$ to 360 $\mathrm{nm}$, in order to investigate the possibility of identifying potentially hazardous ultraviolet light sources through comparison of a spectrophotometer's response to both the standard and the unknown light source. They have also engaged NBS to provide a standard detector in the wavelength region $200 \mathrm{~nm}$ to $320 \mathrm{~nm}$ so that they can evaluate the quality of hazard meter prototypes which are being contracted out and developed by industry for the purpose of developing a capability for remote onsite inspections. Such interactions are essential since ultimately the Occupational Safety and Heal th Administration of the Department of Labor has the authority to levy fines and even close down plants which do not conform to the recommended safety regulations.

(2) Bureau of Radiological Health. Public Law 90-602 has established the Bureau of Radiological Health (BRH) to protect the public health and safety from the dangers of electronic product radiation.* According to section 354 of the Radiation Control for Health and Safety Act of 1968, it is the Bureau of Radiological Health's responsibility

"...to provide for the establishment of an electronic product radiation control program which shall include the development and administration of performance standards to control the emission of electronic product radiation... and the undertaking by public and private organizations of research and investigation into the effects and control of such radiation emissions."

Furthermore, the agency is charged with the mission

"...to plan, conduct, coordinate, and support research, development, training, and operational activities to minimize the emissions of, and the exposure of people to, unnecessary electronic product radiation."

The Bureau of Radiological Health therefore has the responsibility of determining

\footnotetext{
*Defined, in part, as any ionizing or non-ionizing electromagnetic or particulate radiation.
}

standards whereby ultraviolet emitting lamps can be safely sold and distributed. This includes determining the conditions under which a lamp may be a potential hazard to the user and recommending means by which the hazard may be circumvented, e.g., a certain type of safety goggles.

This agency is distinguished from the National Institute of Occupational Safety and Health and the Occupational Safety and Health Administration mainly by its different approach to radiation safety and health. The Bureau of Radiological Health is concerned with the sources of ultraviolet radiation themselves: Do potentially dangerous ultraviolet lamps carry appropriate labels or warnings? Should certain lamps be required to have shielding? Under what conditions are ultraviolet lamps dangerous? The Occupational Safety and Health Administration is concerned with the individuals who are being irradiated by ultraviolet light. Their interest is in the irradiance level at a particular location on the work site. The interaction between these two agencies is fairly strong since they investigate complementary aspects of the same problem, namely, ultraviolet radiation hazards.

The interaction between the Bureau of Radiological Health and NBS has also improved considerably over the last few years. A workship was organized by NBS to discuss our respective mission requirements, research plans, and present and projected calibration capabilities. This was followed up by site visits and an arrangement for the calibration of several deuterium lamp spectral radiance standards in the wavelength range $160 \mathrm{~nm}$ to $300 \mathrm{~nm}$. Because of the very broad nature of their activities (ranging all the way from lasers and ultraviolet lamps to microwave ovens and arc welders), and because of the recent expansion of their responsibilities to include acting as the World Health Organization Collaborating Center for Standardization of Protection Against Non-Ionizing Radiation (see section 2.2.1.1), this interaction is expected to continue to grow.

(3) Consumer Product Safety Commission.

The Consumer Product safety commission was established in 1972:

(a) to protect the public against unreasonable risks of injury associated with consumer products;

(b) to assist customers in evaluating the comparative safety of consumer products; (c) to develop uniform safety standards for consumer products and to minimize conflicting state and local regulations;

(d) to promote research and investigation into the causes and prevention of 
product-related deaths, illnesses, and injuries.

In view of the assortment of "black" lights available for anything from insect traps and science class demonstration kits to "psychedelic" fluorescent light displays, there is clearly a place in the ultraviolet measurement system for the Consumer Product Safety Commission. As yet, there has been no interaction of NBS with this group regarding far ultraviolet radiation measurements or activities.

\subsection{Direct Measurements Transactions Matrix}

A summary input-output matrix has been chosen as a standard technique to be used in all of the microstudies in describing the nature and magnitude of the transactions among the various elements of the measurement system. It is assumed that the reader is somewhat familiar with the technique or has at least read the "macrostudy" report which evaluates and summarizes the input from each microstudy and discusses the information content of the transactions matrix.

\subsubsection{Analysis of Suppliers and Users}

The following standard sector categories are included in the input-output matrix: (1) Knowledge Community--scientific organizations, academic institutions, professional societies, technical publishing organizations (see sections 2.2.5 and 2.2.3). (2) International Metrological Organizations (see section 2.4.1).

(3) Documentary Specification Organizations (see section 2.2.1).

(4) Instrumentation Industry (see section 2.2.2).

(5) NBS (see section 4).

(6) Standards and Testing Laboratories, including those of Department of Defense and any sectors described below, but excluding NBS or any sectors described above (see section 2.4.3).

(7) Regulatory Agencies (see section 2.4.4). (8) Department of Defense (DOD), excluding standards laboratories and any functions already covered above.

(9) Other Federal Government Agencies, for example, the National Aeronautics and Space Administration, National Oceanic and Atmospheric Administration, Energy Research and Development Administration, Department of Labor, Department of Transportation, Department of Health, Education, and Welfare. (10-19) Industrial, Technological, Commercial, or Other Intermediate Economic User/Supplier of Measurement Goods and Services, specifically:
(10) Photochemical Industry

(11) Bacteriological Applications

(12) Environmental Sciences

(13) Medical and Therapeutic Applications

(14) Energy Research

(15) Space Sciences

(16) Laser Development

(17) Photobiology

(18) Plasma Chemistry

(19) General Public.

Table 3 is the actual matrix which applies to the far ultraviolet radiometry measurement system. If one reads across, he finds out how particular sectors distribute their ultraviolet measurement results or capabilities. It tells who used the technology, results, or judgments available from a particular sector. On the other hand, if one reads down, he finds out who is providing input so that a particular industry or other sector can make necessary ultraviolet measurements or make judgments concerning the impact or effects of $u 1$ traviolet radiation. The general information content which is contained in each transaction box is given in the key below the table.

\subsubsection{Highlights Re Major Users}

Details on all of these blocks are contained throughout the microstudy. Rather than defend the individual decisions made in each block, some of which are admittediy somewhat arbitrary, an educated guess if you will, we have decided to concentrate on a few highlights and to point out several significant trends.

One of the first things to notice about the input-output matrix is the correlation between the values placed in the upper left corner (importance or criticality of transaction) and the upper right corner (adequacy of transaction). In very many cases, we find that a transaction is strongly desirable (the number "2" or more) but presently is significantly inadequate (the number "2" or more). This characteristic is also seen by calculating the average transaction quantities of the 163 boxes which have more than a trivial magnitude.

$$
\begin{array}{|ll|}
2.0 & 2.0 \\
1.4^{2.7} & \\
\hline
\end{array}
$$

This average transaction box also indicates that the average interaction is slowly growing and that its present magnitude is a little less than moderate. 
Table 3. The far ultraviolet radiometry National Measurement System illustrated as a direct transactions input-output matrix.

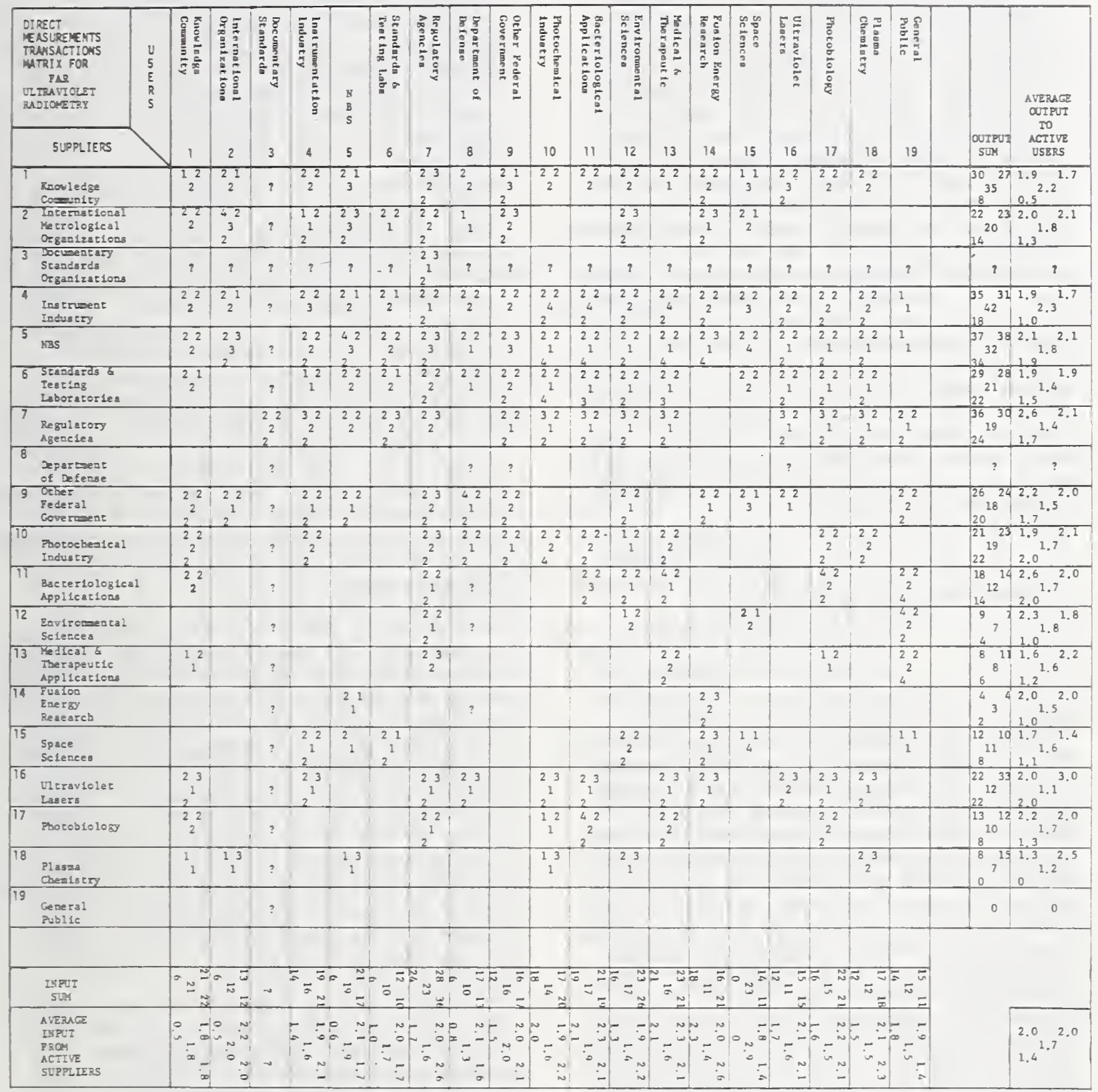

KEY TO MATRIX EUTRIES

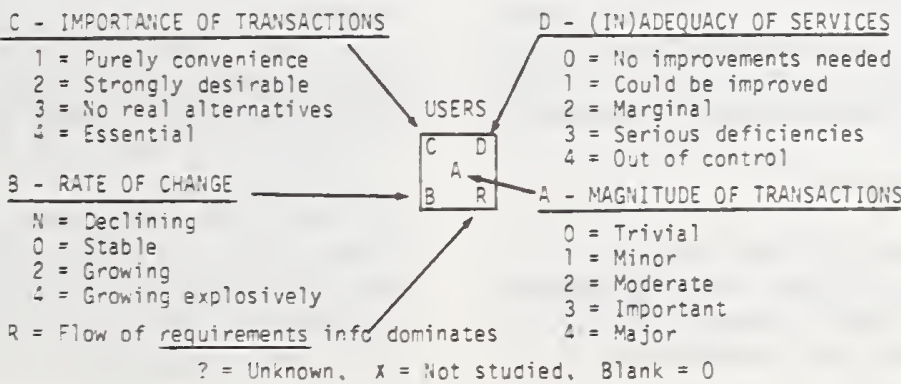


There are several cases where the imbalance in the upper corners is rather marked. For example, let us discuss the transaction indicated by block 12-19 (Environmental Science-general public). Environmental scientists are taking measurements and supplying data on the transmission of solar ultraviolet radiation through the earth's atmosphere. Since even small changes in the transmission have been shown to be a matter of life and death, there is an essential need for communication of this information to the beneficiary or user, in this case the general public. Environmental scientists supply the measurement capability or service, the general public makes direct use of the service, for example, by spending less time outdoors where they might be exposed to cancer-producing ultraviolet radiation* or by organizing programs to cut down on the cause (e.g., atmospheric pollution) of the increased solar ultraviolet transmission. Other examples of where the adequacy of the transaction is much less than it ought to be considering the importance of the transaction are:

(1) Box 2-2. It is most important economically and technologically that different countries interact with each other and have a common measurement baseline.

(2) Box 5-5. It is even more important that the different radiometric efforts within NBS interact with each other, for example, by internal cross-calibrations of source and detector standards.

(3) Box 9-8. Other government agencies like the National Aeronautics and Space Administration, the Energy Research and Development Administration, and the National Oceanic and Atmospheric Administration supply data and services concerning ultraviolet radiation which are critical in matters of national defense, for example, the influence of ultraviolet radiation on the upper atmosphere, missile reentry detection, rocket exhaust studies, and chemical laser systems. (4) Box 11-13, 11-17, and 17-11. These groups have similar needs and goals. Measurement methods developed for bacteriological applications should have considerable impact on photobiology research, and vice versa.

The second thing to notice in the inputoutput matrix is the transactions which are potentially explosive (the number " 4 " in the lower left corner) in regard to growth:

* Outdoor occupations such as highway laborer, lifeguard, field worker, farmer, etc., presentiy suffer a statistically higher incidence of skin cancer [1].
(1) Boxes 10-10, 5-10, and 6-10. The photochemical industry has been doing its own confidential research and development of the uses of ultraviolet radiation and is now just beginning to announce its products and applications through publicity releases and commercialization; by so supplying the results of its own measurement and research capabilities, these companies have a large effect on their own industry (the user in this case). New applications are found, improved methods are developed, and rapid growth takes place in a "resonant" atmosphere. This resonance is only destroyed by either the exhaustion of the potential of the field or by the exchange of misinformation. With respect to the latter, note that the interaction boxes connecting this industry with either NBS or secondary standards laboratories (boxes 6-10, 10-6, 5-10, and 10-5) indicate high probabilities for growth but are characterized by 0 's and l's: trivial or minor present interaction. (2) Box 5-14. - NBS has recently received significant funding from the Energy Research and Development Administration to provide basic data and calibration services required in the national controlled thermonuclear fusion program.

(3) Boxes 11-19, 13-19, 5-11, and 5-13. These emerging industries or technologies are beginning to supply ultraviolet products and services which have direct impact on the health and well-being of the general public. For example, ultraviolet radiation has recently been used in a process which appears to cure psoriasis, a skin condition which afflicts about 8 million Americans. Likewise, ultraviolet radiation is being suggested for use in preventive dentistry (as described later). In view of the serious impact and the consequent concern of the regulatory agencies, NBS interaction is expected to grow proportionally even though the present NBS involvement is minimal (5-11 and 5-13).

A third item of interest is the conspicuous absence of state and local interactions with the National Measurement system. The columns and rows relating to these interactions were deleted from the matrix since they were completely blank, or at least unknown. This is not surprising in view of the fact that the System is relatively young and that even the national regulatory agencies previously described have just begun to interact, having been formed only in the last eight years.

A fourth characteristic to notice is the string of 3 's in the upper right corner of the transactions in column 7 indicating the regulatory agencies as users of the services of various sectors. The suitability of the 
transactions is considered seriously deficient in many cases since the agencies usually require very high accuracies: (a) they have legal authority to close down plants which do not conform to safety regulations and therefore must be very sure of the accuracy of their measurements; (b) they have the responsibility for making specifications which affect many sectors and therefore demand quality input, e.g., how much radiation is dangerous. Because of the effect the regulatory agencies have on radiation health and safety, there is also a string of $3^{\prime}$ 's in the upper left corner of the transactions in row 7 indicating the criticality of the services provided by the regulatory agencies to the various users groups.

Finally, attention is called to the averages which are calculated at the end of each row and column. These are meant to provide some insight into the relative roles played by each sector as either a supplier or user of measurement goods or services. By reading across the bottom row, for example, one gets an idea who are the most important users (integrated over al1 suppliers having a non-negligible interaction). By reading down the last column, one gets an idea of who are the most important suppliers (integrated over a 11 users having a nonnegligible interaction). The indicated sums from which the averages were calculated are also significant since a particular supplier may have little average impact over its users, but may serve many different sectors. The sums indicate this weighting effect.

We see that NBS does not interact very strongly (center number) as a supplier of measurements goods and services on the average. In fact, it is exceeded by the instrumentation industry and the knowledge community and is on the same level with about seven other sectors. This means that, in general, NBS doesn't help anyone a lot and that the users sectors depend more on the measurement system as a whole rather than on NBS. However, NBS does interact in many areas so that when you consider the sums column the NBS relative impact comes out slightly better, although still secondary to the knowledge community and the instrumentation industry. It is my opinion that this patterm should be just the opposite! NBS basic services should not be spread so thin, and they should be more basic or critical to the System (better average values). For example, NBS could put a11 of its resources as a supplier into only two high leverage sectors: the instrumentation industry and the secondary standards laboratories which, by their very nature as commercial establishments, are intended to be omnipresent.

On the other hand, as a user NBS ought to be aware of activities in all sectors, with as good an average interaction with the suppliers as possible. The bottom rows indicate this situation. The average transaction magnitude of 1.9 (moderate) is as good as or better than most of the other users. However, the diversity of the NBS suppliers (as indicated by the sums) could be improved. For example, according to these figures the knowledge community, the regulatory agencies, and the space science community a 11 have a larger overall interaction with the supplier sector than NBS, and there are at least five other groups at about the same level as NBS.

This short discussion should give an idea of the kind of action information that is contained in the input-output matrix. One may take issue with the individual decisions made in each transaction box since it was virtually impossible to base all of them on a simple objective formula. Nevertheless, some basic trends are apparent and are not likely to be changed by a few possible exaggerations or inconsistencies in judgment.

\section{IMPACT, STATUS, AND TRENDS OF MEASUREMENT SYSTEM}

\subsection{Impact of Measurements}

Radiometric calibrations in the visible and infrared have long been one of the continuing, basic measurement services of NBS. Such calibrations represent one of the primary responsibilities of NBS to the needs of measurement technology. In the past few years, U.S. technology has been developing new techniques that require better measurement capability in the far ultraviolet region of the spectrum. The shift toward shorter wavelength follows the extensive scientific exploration of this region that has taken place over the past two decades. The technological importance of this spectral region stems essentially from:

(1) the increasing use of high temperature devices, such as the plasmas being produced in the search for new energy sources;

(2) the industrial potential of far ultraviolet and $x$-ray lasers;

(3) the high energy content of short wavelength radiation with the consequent ability to drive and control chemical reactions; and

(4) the very low diffraction limit of short wavelength radiation.

The problem areas where ultraviolet radiation measurement technology is important 
Table 4. List of Users described in section 3.1.1 together with applicable Standard Industrial Classification Codes (SIC-1972).

\begin{tabular}{|c|c|c|c|}
\hline SIC IMPACT & USERS & SIC IMPACT & USERS \\
\hline $\begin{array}{l}2641,2821,3479,3641 \\
2821,3843,8021,8072 \\
226,3641 \\
2851,3641 \\
2491,2499,2641,3479 \\
806,8011,3641 \\
3811 \\
3641,3646,3662,8411 \\
2741,3641 \\
2753,3641\end{array}$ & $\begin{array}{l}\text { Photochemistry } \\
\text { Thin film production } \\
\text { Tooth decay prevention } \\
\text { Textile dyes } \\
\text { Paint curing } \\
\text { Industrial finishes } \\
\text { Orthopedic Cast hardening } \\
\text { Instant oscillographs } \\
\text { Material degradation } \\
\text { UV photosensitive paper } \\
\text { Counterfeit money detection }\end{array}$ & $\begin{array}{l}3641,3646,3662 \\
3811,3832 \\
3811,3832 \\
3811,3832 \\
3811,3832\end{array}$ & $\begin{array}{l}\text { Fusion } \frac{\text { Research }}{\text { Nontrolionaled ther- }} \\
\text { monuclear fusion efforts } \\
\text { Space Science } \\
\text { Communications } \\
\text { Astrophysics } \\
\text { Skylab } \\
\text { Space Shuttle } \\
\text { Reentry \& rocket exhaust }\end{array}$ \\
\hline & Bacteriological & & \\
\hline $805,806,8011,3641$ & Germicidal lamps in hospi- & & VUV and X-Ray Lasers \\
\hline $2099,2834,3641$ & Environmental Studies & $\begin{array}{l}3811,3832 \\
3399 \\
2831,2833\end{array}$ & $\begin{array}{l}\text { Plasma probes } \\
\text { Isotope separation } \\
\text { Molecular synthes is }\end{array}$ \\
\hline $\begin{array}{l}3811,3829 \\
3811,3829 \\
3811,3829 \\
2099,4941 \\
3829 \\
3829\end{array}$ & $\begin{array}{l}\text { Atmospheric sciences } \\
\text { Ecology and the ozone layer } \\
\text { 0i1 spill identification } \\
\text { Water purification } \\
\text { Water pollution } \\
\text { Smog Gauge }\end{array}$ & $\begin{array}{l}806,8071 \\
3811,3832\end{array}$ & $\begin{array}{l}\text { Tumor therapy } \\
\text { Laser fusion } \\
\text { Photobiology } \\
\text { NBS benchmark experiments }\end{array}$ \\
\hline & Medical and Therapeutic & & Plasma Chemistry \\
\hline $\begin{array}{l}8011,805,806,8071 \\
8011,805,806,8071 \\
8011,805,806,8071 \\
8011,805,806,8071 \\
8011,805,806,8071 \\
806,8071\end{array}$ & $\begin{array}{l}\text { Jaundice treatments } \\
\text { Calcium deficiency } \\
\text { Skin disease treatments } \\
\text { Wrinkling } \\
\text { Drug detection } \\
\text { Medical research }\end{array}$ & $\begin{array}{l}3399 \\
3399 \\
3399\end{array}$ & $\begin{array}{l}\text { Thin film deposition } \\
\text { Plasma arc steel furnace } \\
\text { Recycling of alloys in } \\
\text { steel production }\end{array}$ \\
\hline
\end{tabular}

include national research and development programs as well as major industrial applications. These areas were explicitly included in the input-output matrix and have significant economic and social consequences. That there are diverse and potentialzy explosive areas of impact is one of the main results of this study. Because the many applications and needs were not obvious, at least to us, before this study was undertaken, we have chosen to describe some of these areas, as shown in table 4 , in some detail.

\subsubsection{Functiona 1, Technological, and} Scientific Applications

\subsubsection{Photochemistry}

(1) Thin Film Production. Polymerization of thin fiTms by exposing gaseous monomers to ultraviolet light has been demonstrated at a major industrial research and development center [37]. The technique can be used with a large number of classes of organic compounds and a wide variety of substrates. In operation a polymerization chamber is 
filled with a gaseous monomer at very low pressure and then exposed to ultraviolet light through a transparent section of the chamber. The process, at atmospheric pressure, also can produce films of fused, transparent tefion which ordinarily are sintered and opaque. Applications are anticipated in coating paper products, glass, and metal coils with dielectric, insulating, or protective coatings. Cure rate is dependent on lamp irradiance: too little exposure results in poor quality; too much results in undesirable side effects such as heating of the sample and radiation damage.

(2) Protecting Teeth with an Ultraviolet Polymerized Acrylic Shield. One of the minor uses of acrylic plastics has been as a substitute for conventional amalgams in filling teeth. Now an acrylic plastic has

found another dental use--as a tooth coating which spectacularly cuts down on tooth decay. The material in question is a liquid formulation which hardens to a polymeric resin on exposure to ultraviolet light. The liquid is painted on the biting surfaces of molar teeth with a brush and hardened in about 30 seconds by shining a beam of ultraviolet light onto the coating via a dental mirror. Preliminary tests have shown that the coating, when properly applied, generally stays in place for at least two years and during that time reduces cavities on the protected surfaces by 99 percent.

A group from the New York State Department of Health has tested the material on 4000 Rochester schoolchildren with great success. The product, including the liquid formulation and a special high power, high pressure, air-cooled mercury lamp and associated power supply, is being sold and distributed throughout the United States and Europe. About 20,000 such devices are now in the field. More information is contained in section 4.3.1(1) concerning the economic and social impact of these devices, the ul traviolet radiation measurement problem, and the reaction of the regulatory agencies to possible radiation hazards related to use of the devices.

(3) Textile Dyes and Finishes. Researchers are examining the possibility of replacing the heat used in dyeing and finishing of textiles by ultraviolet radiation. These two textile processes are among the largest energy consuming operations in textile mill processes [38]. The research specifically is designed to explore radiation-induced fixation of flame retardants in fabrics and radiation curing of adhesives in textiles. This is accomplished by shining intense ultraviolet radiation on the materials. It is estimated [38] that radiation curing may require from 10 to 50 percent less energy than for the usual solvent saturated heat cure. Another advantage of the method is that the curing is done without the use of solvents, which are responsible for much of the pollution associated with textile mills [39].

(4) Paint Curing. Mercury vapor lamps are used to accomplish nearly instant curing of industrial coatings by ultraviolet radiation. Paint applied to hardboard, wood, or plastic sheets can be radiation cured in from $2-25$ seconds using ultraviolet chambers developed by the polymers division of a major industrial corporation. A line of radiation-curable coatings also is available for use with the process.

(5) Wood Finishing and other curing Applications. In recent years the ultraviolet cure of unsaturated polyester resins has become a commercial process, particularly in the wood-finishing industry. This is due to the fast cure times, low volatiles, and low investment costs for the ultraviolet systems together with the ready availability and low cost of the resins [40]. It is expected that the technique will also find use in the can, electrical, plastic, paper, and printing industry [39]. Predictions are that as much as 20 percent of all industrial finishes may be cured by some form of radiation (ul traviolet or electron beam) in the U.S. by the end of the decade [41].

(6) Orthopedic Cast Hardening. A casting system which is being marketed commercially [42] uses a webbed glass fiber tape impregnated with an ultraviolet-sensitive plastic resin. A polypropylene stockinette protects the skin, and a web wrap, also of polypropylene, cushions bony prominences. The pliable, slightly sticky tape is applied over the web wrap, shaped to conform to body contours, and rubbed to laminate the layers. Then the resin is hardened by approximately a three-minute cure under an uitraviolet lamp. According to the company, such casts weigh only a third to half as much as comparable plaster casts. Also, they are resistant to breakage, allow air circulation, and can be immersed in water.

(7) Data Acquisition Systems--Instant Oscil lographic Recordings Using UltravioletSensitized Recording Paper. A new graphic data acquistion system, which consists of a direct writing, multi-channel oscillograph with internal plug-in signal conditioning modules, has recently been developed. The unit uses ultraviolet light generated by a cathode ray tube to record on sensitized recording paper through special glass fibers in the tube face. The system produces immediate recordings of analog data 
ranging in frequency from dc to $5 \mathrm{kHz}$ without the need for inconvenient and timeconsuming wet chemical processing.

(8) Material Degradation. A major conglomerate has a large continuing research program on the breakdown by polymerization of wire insulation used in transmission Tines. The overwhelming number of studies done on this type of deterioration suffer from lack of an exact knowledge of the ultraviolet radiation flux (see below).

Solar-simulation accelerated weathering machines are used for testing in every industry whose products are subject to degradation by ultraviolet radiation, such as paints, plastics, dyes, building materials, etc. For example, one large corporation has a very active research program in solar heating and air conditioning for single and multiple dwellings and for small industrial installations. They are testing, among other things, solar reflector and solar absorber coatings in order to come up with a prediction of their lifetime. A spokesman for the corporation describes the tests as follows [43]:

"Will they (the solar absorber coatings) last 20 years? 30 years? We have some coatings life-tested at considerably higher temperatures than we plan to use them. We hope to get a correlation of the high temperature short period which will be equivalent to the long period at the operating temperature."

This, of course, is a common and traditional testing method. However, because of the measurement problems associated with ultraviolet radiation, according to an executive of a major supplier of accelerated weathering machines for the paint, dye, and building material industry:

"Exposure tests result in a chaotic confusion of data with little correlation between laboratory and outdoor tests, and no ability to predict from laboratory tests the expected weather resistance of experimental materials. Thousands of dollars are spent annually in the collection of misinformation due in part to improper evaluation of the effect of the radiation source."

(9) Ultraviolet-Photosensitive Paper. A major chemical company has developed a photosensitive paper based on a dye that forms a permanent blue color when struck by ultraviolet light. Density is controlled by the quantity of incident ultraviolet light. The product requires no chemical or heat processing, and it may be handled in normal roomlight for a few minutes prior to exposure without appreciable fixing.

(10) Fluorescence of Counterfeit Money. A device called the Moneyscanner detects counterfeit money. The device has a high intensity, ultraviolet light under which paper without photochemicals, like Federal Reserve Notes, will not glow.

The Federal Bureau of Engraving and Printing exclusively uses paper made by one particular company. All other papers of comparable grade and quality contain photochemicals. A bill that glows in the bluish light of the MoneyScanner most likely is counterfeit.

\subsubsection{Bacteriological}

(1) Germicidal Lamps in Hospitals, Schools, and Offices. Low pressure mercury lamps have been used for years to destroy airborne and surface bacteria and viruses. Hart reported the extensive use of bactericidal ultraviolet radiation in operating rooms $[44,45]$ and in hospital rooms and wards [44]. In the former, he found that the ultraviolet radiation levels from these lamps reduced post-operative infections by as much as 85 percent [45], al though personnel had to wear skin and eye protection. In the latter, the lamps were used to disinfect the air and furnishings in rooms immediately after discharging patients. Riley [46] has reported how the use of ultraviolet-emitting lamps prevented tuberculos is organisms from spreading, and McLean [47] has reported similar data on the reduced incidence of Asian flu through the use of germicidal lamps.

(2) Germicidal Lamps in Industry. In some applications the entire work area is irradiated in order to destroy airborne mold and bacteria; in other applications, only hoods or ducts are irradiated. Nagy et al. [48] have reported that ultraviolet lamps are 90-99 percent effective in destroying airborne pathogenic organisms. In pharmaceutical houses and biological laboratories, the lamps are used to prevent product contamination. Philips [49] has reported the successful application of germicidal lamps in preventing the spread of pathogenic organisms from a microbiological laboratory to an adjacent office area. UTtraviolet lamps have also been used industrially in the tenderization and preservation of meat. Apparently the $254 \mathrm{~nm} \mathrm{Hg}$ radiation retards mold, whereas the $185 \mathrm{~nm}$ radiation creates an ozone layer around the meat through a photochemical process and protects against microbial growth. 


\subsubsection{Environmental Studies}

(1) Atmospheric Sciences. Dr. Kirby Hanson of the National Oceanic and Atmospheric Administration (NOAA), in an invited paper given at a meeting of the Optical Society of America, described the radiometric needs of oceanographers and atmospheric scientists [50]. Basically, their mission is to study the earth's heat budget, air and water pollution, climatic changes, and the environmental variables of the atmosphere. One of the most important measurements that is at the base of these studies is the heat input, and this depends primarily on solar and terrestrial irradiance, especially in the ultraviolet. Hanson further emphasized two aspects of this requirement which are particularly relevant to this survey:

(a) solar irradiance measurements in the ul traviolet are needed on a global basis in order to construct logical and consistent models for the events which are observed; (b) radiometric accuracies on the order of 0.5 percent to 1 percent are needed in order to keep the time scale for trend measurements reasonable. For example, with a 1 percent accuracy it might take 36 years of data to establish a significant short term trend in the change of ultraviolet transmission through the ozone layer, whereas with 0.5 percent accuracy it would take only 18 years to establish a pattern.

This theme has been reiterated by other members of the atmospheric science community:

"Solar ultraviolet is the dominant source of heating and ionization in the earth's atmosphere and it plays an important role in atmospheric photochemistry.... It dominates the formation of the ionospheric $D, E$, and $F$ regions and establishes the neutral particle composition of the earth's upper atmosphere. Many of the fundamental aeronomical parameters...are obtained from quantitative data on solar uv intensities."

Concerning measurement problems,

"The accuracy of the data on solar ultraviolet fluxes... depends almost entirely upon the accuracy of the photometric standards used for pre-flight laboratory calibration.... Radiometric calibrations of rocket and satellite spectrometers at wavelengths below about $50 \mathrm{~nm}$ are still a major problem since solar uv intensity measurements made by different groups differ by as much as a factor of 2..." Weather prediction, radio communications, the delicate ozone protective blanket-these are typical problem areas which depend upon input from aeronomy and atmospheric sciences. The need for solar irradiance on a global basis points out the need for a consistent measurement system throughout the world and an active measurement assurance program. The simultaneous need for accuracies of 0.5 percent to 1 percent points out the fact that much work on improving far ultraviolet standards and measurement techniques has to be done, by NBS especially, if we wish to serve those scientists who are monitoring the condition of "Spaceship Earth".

(2) Ecology and the Ozone Layer. Johnson [51] and Crutzen [52] first demonstrated that water vapor and especially the oxides of nitrogen emitted as combustion products by supersonic transport (SST) fleets may partially destroy the ozone layer which surrounds the earth and absorbs much of the shorter wavelength ultraviolet radiation. More recently, freon, a common aerosol propellant, has also been linked to ozone destruction in the stratosphere. The Environmental Studies Board of the National Academy of Science and Engineering made a study to determine the possible consequences of such an event. They found that a 5 percent decrease in the ozone concentration would cause a 26 percent increase in broadband ultraviolet transmission and that a 50 percent decrease would cause a factor of 10 increase in ultraviolet transmission. They estimated that the 5 percent decrease in ozone would lead to 8000 additional cases of skin cancer in the United States with about 300 extra deaths. They also expected serious deleterious effects on important agricultural plants and ocean plankton which are so necessary in the ecological balance of our oxygen supply, but were not able to specify details. The recommendation of the Board was to establish monitoring stations at all latitudes to detect any changes in solar ultraviolet transmission and to help evaluate skin cancer data and statistics.

An official speaking in behalf of the NAS/NRC Committee on Photobiology, describes the situation in much the same way as above, and adds

".... The major effect will probably be the catalytic destruction of stratospheric ozone with the resultant increase in ultraviolet light, especially below 230 $\mathrm{nm}$, reaching the surface of the earth. Monitoring stations are being planned to measure, at narrow wavelength bands, the present flux density of uv radiation reaching the surface of the earth at different latitudes. These instmuments, of course, must be calibrated. ....The Committee on Photobiology encourages you to proceed with this most important work 
of developing spectral intensity standards in the near and vacuum ultraviolet region."

These remarks are notably reminiscent of Dr. Hanson's description of the National Oceanic and Atmospheric Administration's radiometric requirements as detailed in the previous section. However, according to a prominent National Aeronautics and Space Administration project leader [53],

"Present day radiometric standards are inadequate by nearly an order of magnitude to permit the interpretation of satellite data in terms of possible long term cl imatological efforts."

(3) 0 i1 Spi11 Detection and Identification Using Ultraviolet Radiation. To facilitate around-the-clock surveillance of harbors and coastal waterways, an oil-spill detection and classification system has been developed under U.S. Coast Guard sponsorship. Selection of appropriate clean-up procedures, a Coast Guard responsibility, hinges in part on identification of the type of oil involved in a particular incident.

Under a contract from the U.S. Department of Transportation, a major electro-optical systems corporation designed and built the optical components and mainframe of an airborne system, from which near 47 traviolet radiation from a $\mathrm{N}_{2}$ gas laser is directed at an oil spill.

The various types of $0 i 1$ produce fluorescence at characteristic wavelengths in response to ultraviolet irradiation. This fluorescent energy is viewed from the aircraft by means of a telescope and focused on a spectrometer. System components scan the spectrometer output and determine the intensity and spectral content of the oil-spill fluorescence. The oil in the spill is identified by comparing the measurement data with readings from laboratory samples of typical oils.

(4) Water Purification Using U1traviolet Radiation. A device for decontaminating water with a combination of ultraviolet and ultrasonic energy has been patented and is expected to have applications for continuous decontamination in laboratories, ships, hospitals, and hotels. By simultaneous use of ultraviolet lamps and high intensity acoustical waves, 100 percent bacteria kill can be obtained in a much greater flow than if either method of radiation were used alone. It doesn't change the taste of water or collect bacteria and viruses on filters that must be periodically removed. The maximum flow rate depends upon the irradiance level of the ultraviolet lamps which must be checked periodically because of possible radiation-damaged optical components in the system.
(5) Water Pollution. Determination of trace mercury, performed by the so-called flameless atomic absorption technique, relies on ultraviolet spectrophotometry. A polluted water sample, for example, is treated with a reducing agent yielding gaseous mercury which is then measured by absorption of its resonance line at $254 \mathrm{~nm}$. One part in $10^{10}$ can be detected. Similar procedures have been developed for arsenic, cadmium, lead, zinc, and many other elements of current interest, as well as a variety of organic pollutants [54].

(6) Smog Gauge. Ozone is produced in the atmosphere through a series of complex photolytic reactions initiated by gaseous pollutants from automobile exhaust so that ozone densities can be used as a "smog gauge". At least one such attempt has been made. A backscattered ultraviolet spectrometer developed by an industrial research group flew on Nimbus 4. Radiation scattered by the earth's atmosphere was detected by the spectrometer, and the data were used to calculate the spatial density of ozone over the earth.

\subsubsection{Medical and Therapeutic}

(1) Jaundice in Infants. In every nursery in every hospital in the United States (and perhaps in the world), there are incubators which are being illuminated by blue or near ultraviolet light. Inside the incubators are premature babies. Twentyfive percent of these babies suffer from jaundice. It used to be an old wives' tale that, to cure such a malady in a baby, one should leave it outside (in the sun) all day. Indeed, light between $300-450 \mathrm{~nm}$ is quite effective in photodecomposing the bilirubin in the body serum which eventually, if untreated, would cause permanant brain damage in the infant. However, 1ittle information on the spectra of the lamps used for these purposes is available and even less is known about possible side effects due to the use of broadband sources. For example, there is concern [55] that the great variation in response to radiation therapy between individuals may be due to the absence or presence in varying amounts of different substances which react to various wavelengths and which can affect bilirubin through photodynamic action. According to a staff member at the Massachusetts General Hospital, pediatricians are just now beginning to consider optimizing the nursery light environment not for maximum visible light (babies are not afraid of the dark) but for the most therapeutic wavelength bands (see also the next section). 
(2) Calcium Deficiencies, Rickets, and Environmental Lighting. As mentioned in the introduction, man is a creature of the sun. His cells adapted to sunlight over hundreds of thousands of years ago, adjusting and timing their activities to its various wavelengths as did his organs. He evolved into an organism designed to respond to the radiation of this remote star.

Then click. On came the electric light less than 100 years ago. The sun was replaced and humankind found that its new stars were incandescent bulbs. The initial effect was to change the time that man was exposed to light. Then, two decades ago, fluorescent lights were introduced on a large scale with a potentially far greater significance biologically since their spectra differ markedly from the sun.

Rickets is a crippling disease which can be caused by a deficiency in exposure to ul traviolet radiation which is necessary for the synthesis of calciferol, a calcifying hormone released into the bloodstream by the skin [56]. Without calciferol not enough calcium is laid down in growing bones. Thus bone deformities were first described in England in about 1650 and were due, we now know, to the extraordinary absorption of solar ultraviolet by industrial smog and coal smoke. In 19th century Europe whole towns could be found afflicted with the disease. We now know this was due to a constant shade condition brought about by a combination of a low sun, at high latitudes in the northern hemisphere, and exceptionally narrow streets and alleys. Rickets is no longer a common problem, but it is easy to forget the lessons of history. Statistics are accumulating $[57,58,59]$ which seem to support the fact that adults, such as the elderly, the infirm, office workers and others who spend a great deal of time indoors suffer from calcium deficiencies. Under these conditions, for example, the fragile bones of the aged grow even more brittle.

Other studies have shown that the use of ultraviolet radiation in a machine shop can produce a positive effect on the health (less catarrhal infections and colds) and bodily functions of the workmen [60]. Similarly, a positive effect on the working ability of school children has been observed [61]. Sexual rhythms and development are also connected to the amount of ultraviolet radiation exposure [59].

Medical doctors and architects are now beginning to consider the addition of an artificial ultraviolet light environment for those people whose circumstances are such that they are exposed to too little sunlight. "Whether we like it or not, light is another thing that physicians must now worry about. The light to which our patients are exposed may be useful, deleterious or, most probably biologically neutral; however, we can no longer assume that it is any of these things without adequate evidence." [62]

(3) Treatment of Skin Diseases. It is estimated that from two to eight million Americans suffer "the heartbreak" of psoriasis, a disfiguring and "incurable" skin disease. The Wall Street Journal [63] and Washington Post have both featured articles describing an experimental treatment utilizing ultraviolet light which is proving to be successful in clearing up the condition [64]. A special drug is taken by mouth followed by exposure of the entire body for 8-20 minutes to a special wavelength of ultraviolet light which happens to be extremely weak in ordinary sunlight, conventional sunlamps, and even the conventional mercury lamps. It is thought that, with periodic retreatment, recurrence of the disease can be prevented. The researchers plan to expand testing of the treatment to a dozen or more major medical centers.

In a similar application, a team of doctors and industrial scientists have been studying the use of an ultraviolet laser and phototoxic drugs for the treatment of various skin diseases. Although much of the work to date has been directed toward the treatment of vitiligo (spontaneous loss of skin and hair pigmentation) which affects an estimated 1 to 2 percent of the world's population, work in the immediate future is expected to be directed toward treatment of warts, fever blisters, and cold sores. Apparently, the drug binds to the DNA in the basal layer of the skin and changes it slightly. After exposure to ultraviolet light, a mild reaction similar to sunburn takes place. Continued exposures to ultraviolet light cause these dark spots to enlarge and ultimately converge. Such treatment is usually hampered by the use of fluorescent ultraviolet lamps whose broadband nature and low intensity make precise exposure time difficult to determine (sometimes as much as two hours per week for as long as two years). The use of ultraviolet lasers is expected to make the treatment quicker and hopefully more precise. The wavelength presently being used in the experiments is $337 \mathrm{~nm}$. Shorter wavelength radiation may be more effective, but this must await the development of convenient laser sources.

(4) Wrinkling and Skin Changes. It is an established fact that ul traviolet light damages proteins that support the skin leading to premature wrinkling [59]. This is espectally common among outdoor workers [1]. 
(5) Drug Detection. Clinical analysis is relying more and more on ultraviolet spectrophotometry. Identification of many drugs, uric acid, proteins, and nucleic acids can be made most conveniently in this wavelength range.

".... Of the various techniques used for this

(drug detection), ultraviolet spectro-

scopy is perhaps most useful. Nearly all

the compounds of interest exhibit meas-

urable uv absorption and can be quickly

detected even at low levels." [65]

A combination of diagnostic techniques involving liquid chromatography followed by ul traviolet spectroscopy has enabled the detection of trace concentrations of metabolites or drugs in blood and urine samples. It is thought that this technique can be applied to disease diagnostics [54] and to the search for disease precursor effects.

(6) Medical Research. The Department of Health, Education, and Welfare awarded 109 grants in 1971 to study the medical effects of radiation, principally in the ultraviolet. The subjects under study include: (a) ultraviolet radiation induced changes in the skin, (b) ultraviolet radiation carcinogenesis, (c) biological effects of environmental radiation, and (d) the effects of ultraviolet irradiation on DNA and RNA.

often, however, the results of such studies are inconclusive because accurate dosimetry is not available. The director of a well-known skin and cancer hospital comments:

"Those of us who have been interested in developing such instruments are immediately struck by the fact that once developed, they need to be calibrated against the reference standard and that there are no efficient and reproducible standards in this field available at the present time.... The need for accurate laboratory and field dosimeters is very great. Clearly before such can be designed and built, reference standards for calibration are vital."

Additional testimony that the effects of ultraviolet radiation are a major field in medical research is obtained from an authority associated with a photobiological

professional society:

".... The effects of uv radiation on cells and biological materials constitute the largest single topic of manuscripts in Photochemistry and Photobiology."

\subsubsection{National Fusion Research}

Our modern industrial society is today struggling with the crucial requirement of ever-increasing energy needs as well as clean and pollution-free sources of energy. Large, government sponsored research efforts to achieve controlled thermonuclear fusion have been mounted in all leading industrial nations, including the U.S. This field has recently experienced a great upsurge of activity with the development of the Tokamaktype plasma machines, which show promise of becoming prototype workable fusion devices. Consequently the Energy Research and Development Administration has embarked on a greatly expanded research and development program in nuclear fusion.

The radiation from the high energy fusion plasmas is particularly intense in the soft x-ray and vacuum ultraviolet regions of the spectrum, and the analysis of this radiation has long been recognized as an important tool for diagnosing the energy balance in the plasma. A survey of the senior staff in charge of the various fusion laboratorles indicates a strong need for an ultraviolet radiometric capability. Some of the comments from these individuals are as follows:

"... We need to know more about impurity concentrations through at least oxygen.

This can best be done in the vacuum ultraviolet but will necessitate knowing more about techniques for absolute calibration of spectrometers.

"...we, therefore, have need for a quantitative measurement of radiance in the vacuum ultraviolet. If standard sources for the vacuum ultraviolet were available we would no doubt purchase them immediately.... In general we are anxiously awaiting the development of radiance standards in the vuv and soft $x$-ray region of the hot plasma spectra.

"...the uv standards program is something I consider absolutely essential.... In my opinion any plasma physics program without absolute intensity measurements is a futite enterprise.

"Radiometric standards are almost non-existent in this (vuv) part of the spectrum...

"A number of Los Alamos programs might benefit directly from the availability of better radiographic standards in the region of about $7 \AA$ to $2500 \AA$..."

\subsubsection{Space Sciences}

(1) Communications Satellites. One field in a stage of rapid deveopment in the present day is space communications technology which involves both commercial and govermmentat development and utilization of satetzites for such purposes as weather forecasting, telecommunications, and earth resources research. Solar simulators have been built for spacecraft and components testing at costs in excess of $\$ 1$ mịlition per 
facility. These simulators are designed to include all the properties of sunlight including color, divergence, spectral distribution, and intensity. Ultraviolet irradiance calibrations are necessary in this field if realistic, worthwhile environmental testing is to be done. According to one of the industrial corporations active in this area:

"We have managed thus far to get along without calibration below $350 \mathrm{~nm}$, but it would be useful for our future work to have standards available for the wavelengths extending down to Lyman a

$(127.6 \mathrm{~nm})$.

(2) Stellar Astrophysics. Dr. J. B. Oke of the California Institute of Technology, in an invited paper given at a meeting of the Optical Society of America [66], described the needs in astrophysics for radiometric standards.

"... They are needed to obtain effective temperatures and gravities of stars to study chemical abundances and stellar evolution. In the case of gaseous nebulas, absolute intensities of emission lines are used for studying the physics and chemical composition of the hot gas ....Better calibrations in the ultraviolet between 120 and $320 \mathrm{~nm}$ are required for space telescope applications."

The director of a large university institute of astronomy relates to us that

"...the analysis of all the spectroscopic data obtained from our rocket flights, from the OSO, ATM, and OAO satellites, and in the future from the shuttle, will depend in the most basic way on the provision of absolute calibration for the detectors."

An industrial aerospace corporation has recently contracted to build the largest National Aeronautics and Space Administration ultraviolet telescope-spectrometer and is most interested in accurate spectral radiance calibration. According to the industry spokesman, since stellar physicists don't know the distance to most of the sources of interest, the space telescope radiometric requirement is not for an absolute standard but for one whose relative spectral distribution is accurate to within about 2 percent throughout the far ultraviolet.

(3) Skylab. Skylab, the first National Aeronautics and Space Administration orbiting workshop, was launched on May 14, 1973 and manned by several teams of three astronautscientists. There were three solar ultraviolet experiments on board, two from the Naval Research Laboratory (NRL) and one from the Harvard College Observatory (HCO). All three experiments depended upon radiometric calibrations: NRL-A used NBS-calibrated deuterium lamps, NRL-B used the NBS synchrotron, and Harvard used NBS standard detectors. In addition several auxiliary calibration rockets were flown during the Skylab mission and also depended upon NBS radiometric calibrations.* According to one of the principal investigators active in the Skylab program:

"The NBS calibration standards program... is of vital importance to our present and potentially our future programs."

Another principal investigator predicts that:

"...although the immediate need is of greatest urgency in connection with Skylab, this need for calibration will continue to exist for follow-on projects involving the far ultraviolet, not only in space research, but also in pure and applied research conducted in ground-based laboratories."

(4) Space Shuttle. After Skylab, there will be an apparent stop in major National Aeronautics and Space Administration space experiments since the development of the space shuttle is the next major National Aeronautics and Space Administration goal. Such a development is supposed to make the utilization of the space environment economically feasible and to provide better opportunities for universities and industries to conduct space research and development. Already, a large aerospace corporation has contracted to fly a payload on one of the first space shuttles. A program director describes their interest in the NBS radiometry program,

"... The facility would consist of a variety of instrumentation for the study of the earth's atmosphere from a low earth orbit. Some of the instrumentation would be designed to operate in the vur down to about $30 \mathrm{~nm}$... . The highest possible absolute accuracy is desired for the solar instruments, and it is mandatory that an on-board calibration scheme be included to provide this accuracy. For the a tmospheric spectrometers, on-board sources for their calibration would also be desirable in addition to ground-based calibration before and after a shuttle sortie mission."

Current plans call for the shuttle to be a fully instrumented flying laboratory which will gather more and better solar and aeronomy data than the current satellite program. Flights are to be scheduled every two weeks in the 1980's. Costs will be

*It was through the Skylab program that NBS received the additional support necessary to initiate a coordinated ultraviolet radiometry program. 
about $100 \mathrm{k} \$$ per hour of $\mathrm{flight--cheaper} \mathrm{than}$ a simple Aerobee rocket flight of a few minutes. Total expenditures for development of the shuttle are currently running $\$ 475 \mathrm{M}$ per year and will increase to over \$1B per year in 1976-1978.

(5) Reentry and Rocket Exhaust Studies. Over the past decade through university, government, and industrial contracting, the Department of Defense has carried on a wide range of innovative programs of great importance to the U.S. space effort, including reentry and rocket exhaust studies. Many of their programs are dependent on the existence of radiometric standards in the ultraviolet as illustrated by the following excerpt from a letter from one of the industrial groups involved in this work:

"We definitely have a need for a calibrated source or detector covering the spectral interval 110-200 nm....Absolute intensity measurements are essential to those experiments where the results are needed to obtain measurements of the concentration of the emitting species or the photon yield of a chemiluminescent reaction. A1so, the measurements of rocket exhaust emission and reentry spectra that we perform must be quantitative for purposes of scientific interpretation or system application.... Participation of NBS in the area of providing better transfer standard sources and detectors below $250 \mathrm{~nm}$ would be of immense value to members of our laboratory and, we feel, to the spectroscopic community at large."

\subsubsection{Far U1traviolet and X-Ray Lasers}

Far ultraviolet lasers are now appearing regularly in the scientific 1iterature. Symposia featuring invited and contributed papers on ultraviolet and x-ray lasers are standard fare at American Physical Society, Optical Society, and IEEE Quantum Electronics conferences. Nonlinear optical techniques have been used to generate coherent emission at wavelengths as short as $89 \mathrm{~nm}$ [67].

Lawrence Livermore Laboratory and others have reported coherent emission in a pressurized $\mathrm{Kr}$ laser at $146 \mathrm{~nm}$ and enhanced gain at 172 $\mathrm{nm}$ when an Ar-Xe mixture is used instead of pure Xe. A cooperative effort between Maxwe 11 Laboratories and Los Alamos Scientific Laboratory is leading to the development of electron beam pumped muzti-gigowatt far ultraviolet lasers. Peak powers of $500 \mathrm{MW}$ at $172 \mathrm{~nm}$ have al ready been measured [68]. Naval Research Laboratory scientists have measured gain at $155 \mathrm{~nm}$ from triply ionized carbon ions (C IV) and are interested in extending their traveling-wave low pressure technique as far as the $\mathrm{x}$-ray region. Lasers are anticipated in $\mathrm{He}_{2}$ near $60 \mathrm{~nm}$ and in $\mathrm{Li}$ and $\mathrm{Na}$ in the $60-80 \mathrm{~nm}$ region. With these lasers and with tripling techniques, in a very short time we shall have a variety of laser related sources throughout the far ultraviolet. The need for power and energy measurements may be expected to follow the same pattern as in the visible region. Fast detectors are being developed at NBS-Boulder and may be applicable. However, they will need to be studied in the far ultraviolet and they will need to be calibrated.

Industrial applications of photochemistry are often hampered by the lack of economical intense radiation sources. However, with the advent of far ultraviolet lasers, this obstacle should be removed within the next few years, and the large scale application of far ultraviolet radiation to trigger and guide complicated chemical reactions may very well be in sight. Areas which appear to be strongly impacted are: (a) photopolymerization, (b) photo-fragmentation, (c) manufacture of pharmaceuticals, (d) biomedical, and (e) petrochemical.

The day is not too far off when the production of certain pharmaceuticals through ultraviolet radiation synthes is will be measured not in grams but rather in tons. Most of the progress in ultraviolet laser technology will probably be in the field of frequency doubling and tripling of visible lasers. However, there is a definite need for radiometric standards which will support the development and industrial application of far ultraviolet lasers. One of the industrial scientists who has been a leader in this field has related to us the following:

"I would iike to support a program" of developing vacuum uv standards. The situation at present is tmizy terribze. I cannot buy a detector which tells me power at $160 \mathrm{~nm}$ that I trust to within $50 \%$.... We are also investigating electron-beam pumped high pressure Xenon lasers. If these lasers work as efficiently as rumored, new industries could be formed to produce chemicals using the light. An example would be ozone. In such manufacturing applications, it would be vital to know the efficiency of each step of the process to within a few percent."

Some specific examples of anticipated industrial applications utilizing ultraviolet lasers are detailed in the following paragraphs.

(1) Photoetching of Microcircuitry-Integrated Circuits. By using a newly. developed electron-resist coating, it is possible to fabricate microcircuit master 
masks with components and spacing of the order of $0.1 \mu \mathrm{m}$ * ${ }^{*}$ The mask can then be used as a photographic master for mass production. When an intense source of far ultraviolet radiation is used, the system is not severely diffraction-limited, and the order of magnitude improvement in resolution is preserved. An industrial spokesman comments on this proposition:

"I cannot emphasize the value and relevance of such possible coherent radiation sources enough.... Soft $x$-ray exposure of photoresists through masks could make the cost of integrated circuits drop by an order of magnitude."

(2) Plasma Probes. The plasmas generated in fusion machines and in high power gas lasers have relatively large electron densities. As the density of a plasma is increased, the plasma frequency also increases. Since the plasma is reflective to radiation of lower frequency than the plasma frequency, diagnostic radiation probes to examine the interior must be higher in frequency than the plasma frequency. Thus it is anticipated that ultraviolet lasers will ultimately be required for diagnostics of very high density fusion devices.

(3) Isotope Separation. Predissociative states have an isotope shift. By tuning to one of the isotopic transitions, one can selectively dissociate the isotopic species. In this way important isotopes such as C 14 and $\mathrm{U}^{235}$ could be produced. Concentrating $\mathrm{C}^{14}$, for example, would make dating much more sensitive.

Experiments at a national laboratory have demonstrated the use of ultraviolet radiation in such applications. Basically one makes use of the high energy content of the ultraviolet radiation for selective photoionization. In order to separate $U-235$ and $U-238$, the experimenters first pass a $590 \mathrm{~nm}$ laser beam through a stream of uranium vapor produced in an oven. This laser frequency matches a transition frequency for bringing $\mathrm{U}-235$ from its ground state to an excited state, but not so for the U-238. Next, an ultraviolet-emitting mercury arc lamp irradiates the stream and selectively ionizes the excited U-235 atoms. A negatively charged electric field then extracts the ionized U-235. Single-step enrichment efficiencies of more than 60 percent have been obtained [69]. The main problem now is capacity or flow volume. One way to improve this is to scale up both the power of the laser and the ultraviolet source.

*Present optical techniques with photoresist coatings are on the scale of $1 \mu \mathrm{m}$.
(4) Molecular Synthesis. By tuning a far ultraviolet laser to excite selected stretching modes of a particular bond, one may break and reform molecules along desired patterns. By such techniques production reactions could be driven.

(5) High Resolution Holography. With a far ultraviolet coherent source, extremely high resolution, high contrast holography would be a possibility. For example, one might anticipate using this technique on very small objects. A university group has already begun on such a project. The general plan is to record holograms on some type of high resolution photoresist (see (1)) and to read out the hologram using an electron microscope.

(6) Tumor Therapy. If an x-ray laser were developed, one would have a pinpoint localization capability for selective destruction of cells.

(7) Laser Fusion. As currently envisioned by some fusion systems researchers, the ideal laser for fusion would have a conversion efficiency of about 10 percent, energy of about $10^{4}$ joules, wavelength in the visible or ultraviolet portion of the spectrum, a high repetition rate, and pulse widthis less than $10^{-9} \mathrm{sec}$. The $\mathrm{Xe}_{2}$ laser at $173 \mathrm{~nm}$ is potentially an extremely powerful laser and is under investigation. Its natural inversion (the lower state of the lasing transition is dissociative--thus no pileup in the lower state to shut off the lasing) leads to an expected overall efficiency of about 30 percent. It also emits in a region of the spectrum where there should be strong absorption by the pellet material [70]. Other gaseous systems such as $\mathrm{Ar}-\mathrm{Xe}, \mathrm{Hg}-\mathrm{Ar}$, and $\mathrm{Hg}-\mathrm{Xe}$ are also being carefully studied for potential application in laser fusion.

\subsubsection{Photobiology}

Far ultraviolet radiation can have many important uses in biology. Years ago it was thought that for photon energies above that required to ionize the atoms, the photoabsorption cross section was a uniform uninteresting continuum. Today we know that this is not the case. Studies in the far ultraviolet indicate that atoms and molecules exhibit many strong resonances in their "continuum" absorption region. These resonances should be found and exploited in biologically significant ways. Many of the resonances which exist in a large molecule are related to specific atoms in the molecule. Thus by tuning the radiation (via a monochromator) to a specific resonance, a particular atom in the molecule can be selectively excited, ionized, or its bonds 
broken. One might even hope to eventually selectively damage particular cells, such as cancerous ones, while doing minimal damage to normal cells.

Such research is not currently being pursued on a large scale, mainly because photobiologists are not accustomed to far uitraviolet measurement techniques and are reluctant to speculate in unfamiliar territory. However, one might assume from the biologists' acceptance of other modern physics techniques such as x-ray diffraction, nuclear magnetic resonance, and electron microprobe analysis that a few good demonstrations would lead to the opening of a new area of research in photobiology.

NBS staff are very much aware of the above described situation and have proposed several benchmark experiments, using the NBS synchrotron radiation facility, which would demonstrate the power and versatility of far u traviolet spectroscopy and radiometry as applied in the field of photobiology. Such important molecules as the polynucleotides, RNA, and DNA are optically active at ultraviolet wavelengths and have chromophores extending into the vacuum uitraviolet. By studying the excitation frequencies and characteristics, one may learn more about the forces binding macro-molecules and the relationships between chemical structure and biological activity. For example, sulfur in a bond position with a high degree of symmetry exhibits a very strong resonance near the sulfur $L_{2,3}$ edge [71]. This resonance can be selectively excited and the sulfur bonding site altered by radiation of the proper frequency. The biological activity of the material (e.g., an enzyme or amino acid component of an enzyme) could be determined as a function of selective ionization of the sulfur atom.

We have received some encouraging responses from the photobiological community as well as positive suggestions for cooperation. A chairman of one of the sections of the American Biophysical Society was most enthusiastic:

"I think that many of the people present at the session would be most interested in your facility. Accordingly, with your permission, I would like to announce the general availability of the radiation facility (SURF II) at the National Bureau of Standards. This will be a very valuable tool for many investigators."

\subsubsection{Plasma Chemistry}

Several commercial applications of arc plasmas will be described in which accurate plasma diagnostics are required for proper controt of the process of interest.
Determination of plasma composition and impurity concentrations, for example, is best done by measuring the relatively strong radiance of ultraviolet light which is emitted by the constituents. Although only three applications are described here in detail, an idea of the variety of applications and the degree of national and international interest on both the part of industry and government is given in appendix $C$. This is a list of companies having an interest in plasma chemistry applications and was prepared by an industrial liaison committee of the subcommission on plasma chemistry of the International Union of Pure and Applied Chemistry. It contains input from eight countries; areas of high interest include synthesis of plastics, extractive metallurgy, vapor deposition and thin films production, and plasma welding, cutting, and etching applications.

(1) Arc Plasma Thin Film Deposition. Arc prasma spraying of ferrite onto integrated circuit substrates offers an economical and rapid method for depositing ferrite films.

This plasma spray technique, which has been commercially developed, can deposit magnesium-manganese ferrites and other materials on a wide variety of substrates at rates that are faster than any other method. Films are reported to be thick, dense, and essentially identical to the bulk starting material. In addition, the non-vacuum process is ideal for manufacturing operations because it eliminates the confining containers required in vacuum techniques. Electron microprobe analyses indicate better than 10 percent tolerance over the entire film surface and 99 percent theoretical density.

(2) Plasma Arc Steel Furnace. A plasma arc furnace that uses powerfut "plasmatrons" to produce five-ton steel ingots reportediy has been prepared by Ukrainian specialists. The plasma arc method of obtaining high quality steels and alloys with preset properties was worked out several years ago at the Institute of Electric Weiding of the Ukrainian Academy of Sciences. Heart of the new installation is a plasmatron that heats gas to a temperature of 10,000 to $12,000 \mathrm{C}$ which melts and refines metal. A Soviet spokesman says the new technique makes it possible to control processes and produce ingots with a desired macrostructure.

Ferro-nickel magnetic ailoys can be made with an oxygen content nearing the sensitivity margin of analytical instruments.

(3) Recycling of Alloys in Plasma Arc Steel Furnace. A commercial-experimental plasma furnace, commissioned at the Chelyabinsk Iron and Steel Works, has produced its 
first 20 melts of particularly pure highalloyed steel. Temperature in the center of the plasma column reaches $18,000 \mathrm{C}$.

High temperature is not the only advantage of the Russian plasma furnace. Metal can be remelted in the furnace without a loss of alloy components. The drawback of conventional furnaces is the high loss of tungsten, chromium, vanadium, manganese, and other valuable components. Losses per ton of metal during remelting range from 20 to 120 kilograms

Soviet scientists and engineers succeeded in obtaining a steady operation of the "plasmatron", producing a column of ionized gases. This column can be seen in a plasma vortex through a light filter. The tungsten pole piece, from which high current flows, is in the center of a cooled pipe. A column of argon gas comes out of the pipe and the atoms immediately lose electrons under the effect of high temperature to become capable of conducting electricity. The plasma column burns noiselessly and steadily and is surrounded with an absolutely pure medium containing no admixtures.

\subsubsection{Economic Impacts--Costs and Benefits}

Having catalogued the types of users who need an ultraviolet measurement capability, we now turn to the question: Are these applications worthwhile? In attempting to answer this question, we will avoid the obvious response that they are good basic science or even that they result in higher quality products. Although we personally think these responses are justified in view of the previous section, nevertheless, we would rather address those who continue to ask the questions: Is it necessary? Is an ultraviolet radiation measurement capability in the national interest? Does it affect our well-being and the common good? Therefore, we intend to briefly discuss examples of both the social and economic impact of the ultraviolet radiometry National Measurement System. First, the economic question will be treated as much as possible with a view toward gaining the maximum leverage in support of the measurement system. A1so, we take the view that, al though numbers are sometimes useful to gauge the efficiency of a supporting operation, they are not always necessary in determining economic leverage. For example, it is clear that without 0-ring elastomer gaskets vacuum technology would have evolved in a completely different way. To try to estimate first of all the total economic advantage of producing demountable, versatile, and rugged vacuum systems as opposed to all glass or welded systems, then the total cost benefit of all industrial and scientific applications that require highly reliable vacuum systems that are conveniently manipulated and varied, and finally to compare these costs with the investment necessary to maintain an 0-ring production and development capability--this would be a meaningless exercise. What difference does it make if the ratio of economic output to invested input is $10^{12}$ or $10^{9}$ ? The point is that such a numbers game is worth considering in detail only when the leverage is less than something like $10^{3}$. Beyond that the leverage should be very clear, and it becomes more efficient for the economist, systems analyst, or planner to merely describe how a certain technology is completely dependent on a particular component (sine qua non) rather than to spend many man-hours searching for figures that will support an already obvious conclusion.

The economic impact of many of the applications described in the previous section are of this type. For example, is it really necessary to calculate what the industrial coating and finishing market is in order to appreciate the prediction that 20 percent of the market will soon depend upon radiation curing (3.1.1.1(5))? Throughout the previous section, such qualitative analyses can be found and are significant. Nevertheless, an attempt is made here to present a quantitative analysis for three areas where numbers were relatively easy to find.

(1) Energy Crisis. The country is now preparing to attack the energy crisis with a crash program comparable to the National Aeronautics and Space Administration space program in the 1960's. Ten billion dollars are being requested for use over the next five years, and our national goal is to have a self-sufficient energy base by 1985 . solar energy and fusion research, both of which have a need for an ultraviolet measurement capability (see, for example, $3.1 .1 .1(8), 3.1 .1 .5$, and $3.1 .3(1))$, are among the priority items in this program.

However, even before our energy problems became well-publicized and "critical", the National Science Foundation and the National Aeronautics and Space Administration had budgeted $\$ 7$ milition for solar energy studies in 1973, and one can surely anticipate even larger budgets for subsequent years. One plan that is being studied is to deploy a large mosaic of solar cells above the atmosphere and relay the absorbed energy to earth via microwaves. Ultraviolet radiometry is required here to study the effects of radiation damage which is expected to be an important factor in the decay and failure of key components in the system.

one could go one step further and begin to analyze the long range effects of an 
energy crisis on our economic system. Already we are feeling the effects of increased unemployment, production delays and shutdowns, decreasing profits, and increasing costs of consumer items from gasoline and heating oil to plastic toys. We do not pretend that an ultraviolet measurement capabi1ity will enable us to avoid these problems, but it is certainly one vital component among many others that will be necessary if the energy crisis is to be permanently solved.

(2) Solar Simulators. We have seen that the cost of building solar simulators can run into the millions of dollars and that ultraviolet calibrations of these are needed at least down to $121.6 \mathrm{~nm}(3.1 .1 .6(1)$ and $3.1 .1 .7(8))$. The problem of realistic solar simulation and accelerated weather testing is complicated by the fact that radiation damage centers (color bodies) formed by exposure at one wavelength may sometimes be destroyed when the sample is subjected to radiation at another wavelength. In addition, activation spectra show that particular wavelengths are most efficient in inducing photodegradation. In the opinion of one of the leading manufacturers of accelerated weathering machines,

"If any real progress is to be made in testing the weather resistance of materials, the exposure standard must be defined in terms of intensity and spectral distribution of incident energy rather than the present parameter of time exposed ....Thousands of dolzars are spent annualzy in the collection of misinformation due in part to improper evaluation of the effect of the radiation source."

Concerning ultraviolet calibrations and its international repercussions, the same individual states in another letter

"...we have recently received information from France that their National Bureau of Standards is considering standardizing the laboratory weathering test. Because of the different light sources in use, it is possible that some instruments may be disqualified if spectral irradiance is not properly specified. We hope they wi11 not disregard the work of the International Standards Organization, as with no generally accepted radiometric standards, more confusion may be generated than dispelled."

(3) Lamp Industry. The economic impact of the lamp industry was discussed briefly. in section 2.2.2.2 (the instrumentation industry). A few of the findings are here summarized. According to the U.S. Census of Manufacturers, the value of shipments of a certain variety of ultraviolet emitting lamps and fixtures was

$$
\begin{aligned}
& 1958--\$ 35.7 \text { million } \\
& 1963--\$ 84.3 \text { million } \\
& 1967--\$ 140.3 \text { million }
\end{aligned}
$$

More than 100 million mercury vapor lamps have been sold for outdoor illumination purposes in the last 40 years. The measurement problem arises in three ways: (a) one wishes to minimize the ultraviolet radiation which then allows more of the available energy to be used for illumination; (b) one wishes to match the artificial light source as closely as possible to natural sunlight either for health reasons, for accurate color reproduction, or for solar simulation; (c) one wishes to maximize or tailor the ultraviolet output, perhaps in certain preferred wavelength bands, for example for medical and therapeutic applications. Accurate ultraviolet radiation measurements are useful, but are they necessary? Yes, say the manufacturers, they are necessary, if only because of economic advantage:

"To substantiate claims against a uv emitting product, accurate measurements are necessary to support the manufacturers case and equally important measurements are necessary to prove that liability exists.

"...the Federal and State measurement laboratories have the power to accept or reject...mizzions of dozlars worth of business. A difference in the unit of light in these laboratories as compared to the manufacturer can cause him to lose large amounts of product sales."

The annual U.S. market for uv-vis spectrophotometers is $\$ 100$ million, with an unfavorable import-export ratio on the order of $4: 1$. The manufacturers are aware of this: "There wil1 certainly be an unfavorable foreign trade impact if electro-optical devices of U.S. manufacture cannot be relied upon to yield NBS traceability quantities."

\subsubsection{Social Impacts}

Much of what is contained in section 3.1.1 deals with the social and human impact of specific programs requiring ultraviolet radiation measurements, e.g., prevention of tooth decay (3.1.1.1(2)) and the monitoring of the stratospheric ozone layer for trends that may affect our environment and health (3.1.1.3(2)). In this section we further amplify on two areas which are of national interest: the energy crisis and health and safety. 
(1) Energy Crisis. Thermonuclear fusion is generally considered to be one of the more promising long range answers to the world's energy needs. With fossil fuels being depleted at a tremendous rate and fuel shortages already being felt, the motivation to produce new sources of energy is indeed strong. It justifies the large investments undertaken by several leading industrial nations, in spite of the enormous difficulties and financial risks involved in striving toward the goal of fusion power.

Fusion research has gone on for almost 20 years, and after periods of near despair and pessimism, recent experiments seem to have put the final goal within sight. The two most critical factors needed to attain fusion are a temperature of nearly 100 million $K$ (comparable to temperatures in the interior of stars) and a containment time (i.e., density $\times$ time) of about $10^{14}$ $\mathrm{cm}^{-3} \mathrm{sec}$ (Lawson criterion). Just a few years ago, fusion machines were a factor of 10,000 or more away from these conditions, but the gap has been closed considerably within the last years to roughly a factor of 100. However, this last factor may prove to be the most difficult to eliminate. The containment times have apparently reached a limit governed by the quenching properties of wall and limiter materials which are evaporated into the high temperature plasma. Present machines can be scaled up in size, at tremendous costs, but a prediction of their performance must first be made, and this depends critically on accurate atomic data, much of it determinable only through vacuum ultraviolet spectroscopy. For example, it is absolutely necessary to know the effect of high energy radiation on the wall materials (radiation damage, photoelectron emission, sputtering rates), the concentrations of impurities (observable as vacuum ultraviolet radiation from highly ionized species), and the transition probabilities and absorption coefficients for these contaminants. Accurate quantitative data, which in most cases depend on ultraviolet radiometry, are needed, and this need cannot be negotiated or compromised especially if meaningful computer simulations are going to be carried out. Approximate estimates and factors of 2 are no longer acceptable. The words of one of the senior staff in charge of diagnostics of one of the Tokamak facilities deserve repeating here:

"...the uv standards program is something

I consider absolutely essential.... In my opinion any plasma physics program without absolute radiation intensity measurements is a futile enterprise."
(2) National Health and Safety. We have described in section 3.1.1 several applications of ultraviolet radiation which impact on the health of the average citizen. Germicidal lamps are used in hospitals, schools, office buildings, and industry to minimize the presence of harmful bacteria and viruses and to prevent them from spreading (see 3.1.1.3(1,2)). An assortment of ultraviolet lamps are used in medical therapy (see $3.1 \cdot 1.4(1,2,3)$ ) and may soon be part of our everyday life as doctors, scientists, and builders investigate the use of artificial ultraviolet light environment for those people* whose circumstances are such that their health is affected by too little exposure to natural sunlight (see 3.1.1.4(2)).

A little bit may be good, but any of us who have been the innocent victims of a summer sunburn know that excessive exposure to ultraviolet radiation can be painful and even incapacitating. Another point must be considered, therefore, in this discussion of social impact: radiation safety. First, does ultraviolet radiation really constitute a hazard to one's health and under what conditions? Second, how often does a source of ultraviolet radiation occur on the labor scene or in our daily life?

Numerous studies indicate that, indeed, ultraviolet radiation can be dangerous. Kinsey et a 1. [72] studied the production of eye damage from arc welding-produced ultraviolet radiation, and Rieke [73] considered that it alone accounted fro 40 percent of all injuries in engineering shops. More often, it is the unshielded bystander who is injured rather than the welder himself who usually wears protective devices. Grim and Kusnetz [74] reported severe pain in workers several hours after a 10 second exposure to radiation from an arc torch that generated an intense flame $20-30 \mathrm{~cm}$ long. Powell et a1. [75] studied hazards from both laboratory and industrial plasma torches and found the output of these sufficient to cause eye and skin irritation on long exposure. There have also been many studies on photokeratitis due to ultraviolet irradiation of the eyes [76-79]. Cases of dermatitis and erythema have even been reported from ultraviolet radiation below $320 \mathrm{~nm}$ produced by fluorescent lamps used for general lighting purposes $[80,81]$.

*This group might include, for example, office workers who arrive and leave in darkness, night workers who sleep during the day, and people who live in very cold climates. 
Aircraft workers

Barbers

Bath attendants

Brick masons

Burners, metal

Cattlemen

Construction workers

Cutters, metal

Drug makers

Electricians

Farmers

Fishermen

Food irradiators

Foundry workers
Furnace workers

Gardeners

Gas mantle makers

Glass blowers

Glass furnace workers

Hairdressers

Herders

Iron workers

Li feguards

Lithographers

Metal casting inspectors

Miners, open pit

Nurses

$0 i 1$ field workers
Pipeline workers

Plasma torch operators

Railroad track workers

Ranchers

Road workers

Seamen

Skimmers, glass

Steel mi11 workers

Stockmen

Stokers

Tobacco irradiators

Vitamin D preparation makers

Welders
Cases have also been reported where the topical application or the oral administration of certain drugs or chemicals causes the skin to become hypersensitive to ultraviolet and visible light. In many cases the photosensitizing ability of a drug has been discovered only after its acceptance for clinical use. Not too long ago, there was a well-known after-shave lotion that was finally identified as causing severe allergic reactions when activated by sunlight. On the other hand, such reactions can be used therapeutically as we have seen in the treatment of psoriasis and vitiligo (see 3.1.1.4(3)). Pathak [82] lists various agents implicated in the photosensitivity reactions of the skin and showed their therapeutic uses. However, care must be exercised here also. For example, the photochemical conversion of provitamin $D$ to the active compound by uitraviolet irradiation is a well-established fact. Gorter [83] found that with $297 \mathrm{~nm}$ radiation a daily dose of $2 \times 10^{4} \mathrm{ergs} \mathrm{cm}^{-2}$ was required to cure rickets in children. However, according to standards set up by Coblentz et al. [84], this dose amounts to 50 percent of the minimal erythemal dose (MED) over a smal1 skin area.

The connection of skin cancer with ultraviolet radiation damage is now beyond controversy [85-89]. According to Epstein [85],

"...atthough there is some question about

the carcinogenic spectrum in human skin

cancer, there is no controversy about

experimental cancer produced by uv

radiation."

Solar radiation is a clear factor in the etiology of skin cancer according to

many researchers $[90,91]$, including
Dr. Frederick Urbach $[92,93]$ chairman of the Temple University Skin and Cancer Hospital. In connection with this, experiments have shown that ultraviolet radiation induces alteration of RNA and DNA synthesis $[85,94-96]$.

Ultraviolet radiation can be dangerous, but does it occur in everyday life in such harmful doses? The answer is yes. Solar radiation is probably the worst offender. Numerous statistical studies have been done such as the one by MacDonald [97], who found that the prevalence of carcinoma in E1 Paso County, Texas, where the sun shines during 80 percent of the daylight hours, was eight times higher than in Hartford, Connecticut, where the sun shines 50 percent of the daytime hours. Sunlight, specifically the wavelengths from about $290 \mathrm{~nm}$ to $325 \mathrm{~nm}$, has been shown to be far more important than aging in producing wrinkles and skin changes [98-100].

Ultraviolet radiation is also common indoors. The Food and Drug Administration has estimated [101] that over 20,000 sun 1 amprelated injuries alone have been treated in U.S. hospital emergency rooms during the two year period 1973-1974. This number includes only emergency room statistics and does not include those injuries treated by other medical personnel. Outside the home, ultraviolet radiation can be found as a by-product of welding and plasma torch operations, photoelectric scanning, and hot metal operations. It is present as an artificial source for the following applications:

(a) germicida 1 lamps in hospitals $[44,45]$, biological laboratories [49] and schools;

(b) illumination;

(c) advertising; 
(d) crime detection [102];

(e) chemical synthesis and analysis;

(f) photoengraving;

(g) food [103], water [104], and air [46-48]

sterilization;

(h) vitamin production;

(i) medical diagnosis, e.g., dermal and

scalp disorders;

(j) ultraviolet lasers;

(k) fluorescent panels;

(1) mineral identification;

... and others. For example, some occupa-

tions which are potentially dangerous due to

ultraviolet exposure [105] are shown in

table 5.

Finaliy, one more estimate of the frequency of industrial ultraviolet exposure is contained in the National Institute for Occupational Safety and Health radiation safety criterion document [1]. A poll of the Chicago metropolitan area was taken and the number of people exposed only to arc welders and ultraviolet radiation air purifiers and sanitizers in the work location was determined. Extrapolating this number to the whole U.S. population, it was estimated that about 320,000 workers are daily exposed to potentially harmful ultraviolet radiation doses from just these three types of sources.

Again, a little bit may be good, but too much is harmful. As long as ultraviolet radiation is going to be a part of the daily scene in our lives, a measurement capability will be absolutely necessary not only to provide for better health and medical treatment but also to guarantee our safety.

\subsection{Status and Trends of the System}

Far ultraviolet radiation, of interest primarily to scientists in the 1950's and 1960 's, is beginning to be applied now in many different technologies. Photochemistry, bacteriology, environmental studies, plasma diagnostics, fusion research, biomedical science, photobiology, space physics, ultraviolet lasers, microcircuitry--all are fields currently affected to some degree by the presence (or absence) of an ultraviolet radiation measurement capability. The following technology forecast, in the form of fictitious publicity releases, shows how some of these applications might conceivably intensify, broaden, and mature in the years to come.

Item 1: Oceanographers have recently occupied the world's first permanent undersea laboratory in the Gulf Stream off the United States East Coast. Mindful of the harmful effects suffered by U.S. astronauts in the manned orbiting laboratory experiments due to insufficient exposure to sunlight, the Navy engineers have installed artificial light supplemented by special ultraviolet lamps which are used to simulate actual solar irradiance. The calibration of these units was made possible through cooperation between NBS, the National Institute for Occupational Safety and Health, and Devices, Inc., producer of the lamps.

Item 2: Solar energy is now being tapped 24 hours a day through an orbiting solar cell network developed and produced by the electric power industry. Energy is beamed back to earth from a synchronous orbit via microwaves. Far ultraviolet radiation damage to the optical components has been minimized and energy transfer optimized through the identification and elimination of contaminant absorbing monolayers present because of outgassing components. Plans are now being considered to scale up the operation to such an extent that the facility may be visible in the evening sky even to the naked eye.

Item 3: Tunable vacuum ultraviolet lasers are being utilized in high sensitivity photoionization mass spectrometers to detect trace quantities of various organic and inorganic constituents in skin tissue, breath samples, perspiration, etc. The monochromatic nature of the high intensity lasers allows energy-selective ionization followed by conventional mass spectrometry. The method is being used as a medical diagnostic in several major hospitals and is expected to soon replace the conventional gas chromatograph because of $i$ ts large advantage with respect to analys is time. In addition, with the increased sensitivity as well as the simpler spectrum, precursor effects are being identified and studied in the hope that diseases such as cancer can be predicted and effectively prevented.

Item 4: An additional service from the optics and spectrophotometry industry is now available. Standard far ultraviolet sources and detectors certified by NBS are being used to provide economical spectral reflectance curves for holographic and conventional dispersion gratings. This has resulted in an order of magnitude improvement in grating production quality control and has to a large extent neutralized the impact of foreign made gratings and spectrometers on the world market.

Item 5: Far ul traviolet technology, handicapped up to now by the unavailability of transparent window materials below 110 
$\mathrm{nm}$, is expected to increase greatly due to the recent development of a multilayer structure of thin films which have transmission limits extending as far as $10 \mathrm{~nm}$ and which are capable of withstanding a 1 atm pressure differential. Scientists at NBS have described this breakthrough at a...

This is indeed an exercise of the imagination, but we all know that time seems to move more quickly these days and that there is an accelerating decrease in the time lag between scientific discovery and usefur application. This natural acceleration is enhanced even more by the identification periodically of various "crises"--Sputnik, balance of trade deficit, pollution, and now the energy crisis. The "fiction today, fact tomorrow" phenomenon sometimes can make technology forecasts outdated even as they are written. For example, I had originally intended to include in the previous fictitious publicity releases a description of an $x$-ray laser being used for medical treatments when the following was brought to my attention:

"Battelle-Columbus scientists currently are conducting a study for the North Central Health Foundation...to develop a probe device intended to deliver lasergenerated soft $x$-rays to surface tissue or to locations in the body that presently are difficult to reach with conventional techniques.... At present, large quantities of $x$-rays are radiated from a highly nonequilibrium plasma in the form of spectral lines. This $x$-ray source is said to be only one step away from being an $x$-ray laser. The final step...will be to establish the conditions for line amplification." [106]

or how about the "advertisement" reproduced in figure 7 which was published in a technical journal [107]?

What about the measurement system that supports and interacts with these fields? We have seen that primary radiometric standards, both sources and detectors, exist throughout the ultraviolet spectrum and are fairly well developed. However, they are usually too complicated or specialized to be applied in practical situations. Transfer or secondary standards are absolutely necessary and serve as the essential link between the central standards organizations, the calibration laboratories, documentary specification institutions and regulatory agencies, and the ultimate users.

Transfer sources are not very well developed in the far ultraviolet. Where they are available, radiometric uncertainties are generally not less than 6 to $10 \%$. This is adequate for many users, but quite inadequate
GUARANTEED DEMONSTRATION OF

COMPRESSION NEUTRONS

THE KMS OPTICAL SYSTEMS DIVISION OF KMS INDUSTRIES INC IS OFFERING FOR SALE, FOR $\$ 8,000,000$ A LASER FUSION SYSTEM

The system is able to vary the pulse shape at different pulse lengths. Also included in the system are the following:-(1) a unique illuminetion system. (2) a targe alignment focusing system. (3) significant diagnostic equipment including:Totel neutron vield

Target ion spectra
Total ion yield

Totel $X$-rey yield

Time integrated. spacially resolved

$\mathrm{X}$-ray emission (target compression monitor)

X-ray spectrum (both line and con(inuum)

Target coupled energy

Laser optical pulse calorimetry

(both incident and reflected energy)

Target blest wave energy.

KMS INDUSTRIES states through its KMS FUSION subsidiary it is offering LASER FUSION TARGETS for sale at a price of $\$ 250.000$. The price is inclusive f:-

(1) The right to obtain pellets for three years.

(2) An initiel 10,000 selected pellets from a KMS FUSION catalogue. The Additional pellets for sale are at the rate of $\$ 1.00$ to $\$ 3,00$ pach

If a customer acquires:-

(1) The KMS OPTICAL SYSTEMS laser fusion system.

(2) Pellets from KMS FUSION.

(3) Hires KMS INDUSTRIES to take a time and materials menagement contract for installation services, KMS FUSION will guarantee the demon stration of compression neutrons.

KMS INDUSTRIES is also willing

(1) To approve other laser infusion systems which completely meet the SY KMS FUSION.

(2) If KMS INDUSTRIES received the installation manegement contrect to guerantee that the specifications continue to be met efter installetion.

(3) And if KMS FUSION will guarantee the demonstration of the production of compression neutrons from the KMS FUSION epproved laser fusio system

Figure 7. A copy of a magazine advertisement illustrating a technology forecast with respect to laser fusion systems.

for several high impact user groups, who require $1 \%$ or better uncertainty limits. Below $165 \mathrm{~nm}$ there are currently no reliable sources which are suitable as secondary standards. However, high pressure noble gas stabilized arcs are being studied and seem to be a good candidate for servicing the $110-300 \mathrm{~nm}$ region. Below $110 \mathrm{~nm}$, one would really prefer a low pressure source (in order to avoid an inconvenient differential pumping system) such as transition radiation [108]. Currentzy, to our knowledge, there are no plans to develop such sources.

Transfer detectors are fairly well deve1oped in the far ultraviolet although recent service requirements from the user community seem to demand increased research in this area. There is a need for extension of the calibration range (currently 19-320 $\mathrm{nm}$ ) to both shorter $(5 \mathrm{~nm})$ and longer $(400 \mathrm{~nm})$ wavelengths. There is also a need for higher accuracy, especially in the wavelength range $110-400 \mathrm{~nm}$ where $1 \%$ uncertainties are required.

The significant thing to observe at this point is the lack of suitable interaction 
among almost all of the areas identified as having a measurement need or responsibility and the suppiiers of primary and transfer standards. So far, only the space industry has been adequately served, as can be seen by referring to the input-output matrix in table 3 . This is one of the key findings of this survey. The reasons for the lack of interactions seem to be the following:

(a) basic standards are necessary before regulatory agencies or commercial calibration laboratories can be set up, and in general, these standards are still in the research stage;

(b) those standards that are available have not been sufficiently publicized because no one seems to have a clear view of the impact and needs of the various areas of application.

In short, the measurement network is still in the development stage. The lack of a more rapid evolution is due in large part to the lack of adequate standards. If the interactions among the standards community and the rest of the measurement system are not improved, it is doubtful that some of the fields with extraordinary social and economic potential such as environmental lighting, medical therapy, and photopolymerization applications will continue to develop.

\section{SURVEY OF NBS SERVICES}

\subsection{The Past}

Until 1971, ultraviolet radiometric activities at NBS were carried out only in the Optical Radiation Section (232.04). A description of the size and magnitude of the ultraviolet portion of the total radiometry and photometry effort of this Section is contained in the microstudy Radiometry and Photometry and will not be repeated here. In general it can be said that ultraviolet radiation was viewed in this case as the lower limit of the visible spectrum, and with regard to the Optical Radiation Section's priorities, ultraviolet radiometry was certainly secondary to visible radiometry and photometry.

In 1971, the Far UV Physics Section (232.03) of the Optical Physics Division made its first ultraviolet detector calibrations for the National Aeronautics and Space Administration. Their facility was an outgrowth of the Section's ongoing basic research which depended upon stable and reliable detectors. This ad hoc facility grew some roots when in 1972 both the Far UV Physics and Plasma Spectroscopy (232.07)

Sections, with National Aeronautics and Space
Administration funding, accepted the responsibility of developing ultraviolet detector and source standards which would provide for a common baseline for the solar physics experiments on board Skylab, the first manned orbiting scientific workshop. Through continuous outside funding, the effort in these two sections has been built up to its present size, a full-time professional staff of four. Four workshops were organized during this period by the staff concerning calibrations of space experiments. In addition, one of the staff acted as the overall coordinator of ground-based radiometric calibration programs for Skylab. However, a weakness of the NBS far ultraviolet radiometry program should already be apparent: total dependence on National Aeronautics and Space Administration funds results in total cormitment to their goals and deadlines. Under these circumstances, responding to other measurement priorities as expressed by other sectors of the system is a slow and indirect business, mostly a matter of adaption and "on-the-side", "when-there-is-time" calibrations and experiments.

\subsection{The Present--Scope of NBS Services}

\subsubsection{Scope of NBS Services}

A short description of the various calibration methods has already been given in section 2.2.2.1. This section concentrates more on what services are or will be available from NBS. In its short span of existence, this work has already found many applications in the nation's space program, and more recently in plasma and fusion laboratories, in national health and safety programs, and in several other measurement sectors. The ultraviolet radiometric calibrations of virtually all current space physics experiments may be traced to the NBS program.

The NBS program on far ultraviolet radiometric calibrations is carried out primarily in the Far UV Physics (232.03) and the Plasma Spectroscopy Sections (232.07), and to a somewhat lesser extent in the Optical Radiation Section (232.04). All three Sections are in the Optical Physics division, Institute for Basic Standards. The program consists of several complementary components and makes use of absolute detectors as well as standard radiation sources. That there are several avenues of research at NBS is a good thing since none of the calibration methods is without its drawbacks and uncertainties. A survey of the current and projected NBS capabilities (for the next 
two or three years) is given in figure 8 .

(1) Detectors. Two basic detector configurations suitable for radiometric calibrations have been developed and tested in the Far UV Physics Section. Both types are small, relatively rugged and require only conventional electronics for use; they thus may be used to transfer a radiometric calibration from NBS to outside calibration laboratories or other users. About 60 such detectors have been calibrated during the past two years, forming the radiometric base for much of this country's solar and upper atmosphere physics programs as well as portions of the astronomy program. In addition, several calibrated detectors have been supplied to scientific laboratories in England, France, and West Germany.

The two configurations are selected variations of the simple vacuum photodiode. A windowed type with a low work function photocathode is calibrated from about $120 \mathrm{~nm}$ to about $260 \mathrm{~nm}$, and a windowless type, of NBS design and fabrication, with a high work function photocathode, is presently calibrated from about $19 \mathrm{~nm}$ to about $120 \mathrm{~nm}$. The quantity calibrated is the absolute quantum efficiency, or the number of photoelectrons per incident photon, as a function of wavelength.

Master photodiodes of each type are calibrated by means of a special thermopile which, in turn, is calibrated with a rare gas ionization chamber. Comparisons are periodically made with calibrations of the same thermopile using either a total irradiance source or calibrated $253.7 \mathrm{~nm} \mathrm{Hg}$ source. The master detectors thus calibrated are used to transfer the calibration to outgoing detectors.

The estimated probable error in the calibrations of outgoing detectors is 6-10 percent in the case of the windowed photodiodes and 8-10 percent in the case of the windowless photodiodes. The apparent change in the calibration of windowed photodiodes in use outside NBS for a period of eight months was less than 2 percent. Windowless photodiodes have not yet been returned for recalibration, but in-house samples have demonstrated reproducibilities of 2-3 percent over a few months and 10-20 percent over two to three years.

Plans for the next two or three years include the establishment of a facility at NBS-SURF (Synchrotron Ultraviolet Radiation Facility) for the extension of the short wavelength calibration of windowless photodiodes to about $10 \mathrm{~nm}$. This facility will also be used to study the environmental effects on the stability of a calibration at these shorter wavelengths. Initial measurements in this program have already been taken. Extension of the high wavelength

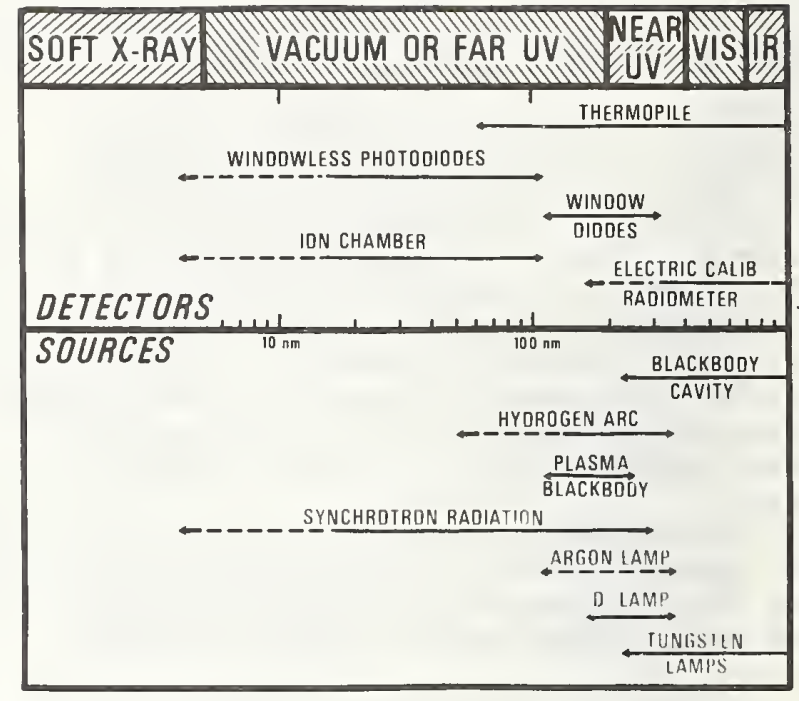

Figure 8. Current and projected NBS standard source and detector capabilities. The dashed lines indicate near term projections.

limit of the window photodiodes to about 400 $\mathrm{nm}$ is also being considered due to a high degree of interest expressed by the biomedical field and the regulatory agencies, the Occupational Safety and Health Administration in particular.

(2) Sources. The sources currently under development for radiometric applications are the wall-stabilized hydrogen arc and the NBS synchrotron, both primary standards, and the deuterium lamp and the argon "mini-arc", both transfer standards.

The wall-stabilized hydrogen arc plasma radiates a calculable and strong continuum in the vacuum ultraviolet. The spectral radiance of this source has been checked for consistency against a number of other standard sources and techniques. It has been compared to calibrated tungsten strip lamps for wavelengths above $250 \mathrm{~nm}$ : the measured and calibrated hydrogen continuum intensities were found to agree within 5 percent. Below $250 \mathrm{~nm}$ it has been checked against blackbody-1imited lines produced by another stabilized arc (plasma blackbody) seeded with impurities of carbon, nitrogen, and oxygen atoms: consistency with this technique has been achieved within 5-10 percent. Based on these results, the hydrogen arc is considered to be operational as a spectrai radiance standard for the range from $130 \mathrm{~nm}$ to $350 \mathrm{~nm}$. The method is to be extended in the next few years down to about $50 \mathrm{~nm}$.

Since the arc and its associated power supplies are rather bulky and difficult to 
handle, preliminary work is also underway on the development of secondary or transfer radiation sources. Commercial deuterium arcs have been found to perform this function quite satisfactorily (except for gradual aging effects) in the range from $165 \mathrm{~nm}$ to $350 \mathrm{~nm}$. During the last four years, the spectral radiance of a total of 47 deuterium lamps and one Krafft-Roessler mercury lamp has been calibrated for outside users, and more calibration requests are anticipated. Calibration uncertainties are estimated to be $\pm 10 \%$ due in large part to lamp alignment difficulties and some variability in the output of the lamp from one lighting to another.

As of January, 1976, deuterium lamps calibrated for spectral irradiance in the range 200 to $350 \mathrm{~nm}$ have also become available due to a collaborative research effort between the Plasma Spectroscopy and Optical Radiation Sections. About 20 calibrated lamps were issued during the first five months following the announcement of this service. Uncertainties are estimated to be $\pm 6 \%$.

Current research is concentrating on the development of a more versatile (wider wavelength range, higher intensity) and more reliable (ease of alignment, no aging effects, better stability) transfer source. The argon mini-arc, a one atmosphere pressure noble gas discharge, is being calibrated with respect to spectral radiance between 115 and $350 \mathrm{~nm}$. Although slightly more complicated than the deuterium lamp, it is anticipated that this radiation source will eventually replace the deuterium lamp source in far ultraviolet applications requiring uncertainties of about $5 \%$. A spectral irradiance calibration facility in the far ultraviolet is also planned for development; both the deuterium lamp and argon mini-arc will be calibrated to serve as spectral irradiance standards below $200 \mathrm{~nm}$ in the next few years. The argon mini-arc will be tested also between 200 and $350 \mathrm{~nm}$ where it may prove suitable as a high intensity irradiance standard much stronger than both the deuterium lamp and the tungsten quartz-halogen 1 amp.

The NBS synchrotron radiation facility is also being used as a calculable source for far ultraviolet calibrations. In the recent conversion of the NBS electron synchrotron into an electron storage ring source, particular attention was paid to making accurate determinations of the operating parameters. The energy and radius of the orbiting electrons can be determined to within 0.1 percent while the orbiting current can be determined to about 3 percent (by means of radiometry in the visible region). In its new form the NBS synchrotron facility provides a smooth calculable continuum of useful radiation from about $4 \mathrm{~nm}$ in the soft $x$-ray region into the visible. Its highly collimated output varies slowly in time with a time constant of the order of one hour. It is compatible with ultrahigh vacuum systems and is capable of determining the polarization response of spectroscopic instruments. The spectral radiance emitted may be determined to a precision of better that 5 percent.

It is intended that the facility will be made available to guest workers on a fee basis. Several users meetings have been organized and a booklet describing the characteristics of the storage ring facility has been distributed to potential users.

(3) Interactions of NBS with the National Measurement System.

(a) Standards Laboratories. NBS has provided standard detectors to standards groups both in England (National Physical Laboratory in Teddington), in Germany (Max-Planck Institut für Physik und Astrophysik in Munich) and in the United States (Stanford University in Palo Alto) for intercomparison purposes. The results of the international comparisons are not yet completed, but are of great interest since the preliminary results obtained by the Munich group in comparing detectors calibrated by several independent European laboratories show large discrepancies (up to a factor 2). Comparisons of detectors calibrated by NBS and by the Stanford group are excellent and result in consistency to within the calibration uncertainties.

NBS is also involved in standard source intercomparisons. In a completed effort, the Hamburg DESY synchrotron ultraviolet source was essentially compared with the NBS hydrogen arc source through a common deuterium lamp transfer standard [31]. Future intercomparisons with the central standards laboratories in England, France, and West Germany are being planned in cooperation with three guest workers from these countries who spent one year each at NBS and assisted in setting up part of our ultraviolet radiometry program.

NBS has aiso recentiy cooperated with a commercial standards laboratory in order to make available standard lamps of spectral radiance and irradiance in the far ultraviolet. Nevertheless, the magnitude and intensity of this type of interaction is considered to be much too smali in view of the needs of the various measurement sectors for uniformity in ultraviolet radiation diagnostics.

(b) Regulatory Agencies. NBS interaction with regulatory agencies is on the increase, and, in fact, it is through such interaction that the commercial standards laboratory just 
referred to first became aware of the available NBS services. Two mini-workshops were organized at NBS in order to exchange information with representatives from the National Institute for Occupational Safety and Health and the Bureau of Radiological Health. This was followed up by site visits, calibration of standard lamps for each group, and a service contract to evaluate, through the use of standard detectors, a commercially produced "hazard-meter" which the National Institute for Occupational Safety and Health was considering recommending to the Department of Labor as a monitor of ultraviolet exposure in the work environment. This is obviously a high leverage situation for NBS. If it can be definitely established that the meters meet specifications--and this depends on NBS having the necessary radiometric capability--it is possible that every machine shop and every industry 1 isted previously in table 5 will need to purchase such an instrument. Also, there will be created a market for accessories such as standard sources of spectral irradiance to periodically check the operation of the unit.

(c) Instrumentation Industry. A very strong interaction exists between NBS and one particular manufacturer of photodiode detectors. NBS did the research and development in cooperation with this industrial group which now furnishes all the detectors calibrated by NBS. The interest of this group was high enough that they even undertook an internationa 1 round-robin intercomparison of detectors among various space science groups who were using NBS calibrated detectors. The results were reported at a calibration workshop [35] and provided information with regard to aging effects, uncertainties associated with calibration transfers, and steps that ought to be taken toward a measurement assurance program. Unfortunately, such interactions are rare, although several optical companies have been in periodic contact with NBS concerning our standard source developments and have expressed some interest in marketing our final product, especially if it has universal appeal. As close as we can ascertain, this means that it must have a wide wavelength range of applicability and it must be intense, calibrated, convenient, and cheap.

(d) Basic Data and Materials. NBS generates and evaluates basic data which are necessary in the analysis of many ultraviolet emitting systems (see section 2.2.3). Transition probabilities, oscillator strengths, reaction rates, and photoemission cross sections are measured or calculated. In addition, a data center on atomic transition probabilities has been established to review and critically evaluate the available literature. Although such basic research can be found in many places throughout NBS (and the world), most of the NBS work which utilizes ultraviolet radiometry to generate such data is centered in the Far UV Physics Section (storage ring) and the Plasma Spectroscopy Section (wal1-stabilized arcs and theta pinch). The Far UV Physics Section also has done optical reflectance and transmittance research in the far ultraviolet using the NBS synchrotron. However, standard filters or materials in this wavelength region have not been researched and are not available as an NBS service. Such services are available commercially, al though the calibrations are not NBS traceable.

(e) Dissemination Network. Some, but not all, of the radiometric services available in the far ultraviolet are listed in Special Publication SP-250: Calibrations and Test Services of the National Bureau of Standards. An effort is being made to improve on this situation and to include alz of these services in the next edition.

\subsubsection{Users of NBS Services}

Table 6 illustrates the impact areas and users so far served by the five year old NBS far ultraviolet radiometry program.

It is clear from this table that so far the primary consumers of our far ultraviolet services are the space scientists who need absolute detectors and sources to determine the response of spectrometric optical systems. These dominate by about 10:1 over the combined number of all other current consumers. However, this statistic is due mainly to the chronology of how the measurement system has evolved, as alluded to several times earlier. Research and scientific exploration (National Aeronautics and Space Administration in the 60's) forced the development of advanced technology which, in turn, stimulated and made possible new applications of that technology. Some evidence that we are entering this latter stage is seen in the fact that the number of non-space oriented far ultraviolet calibration requests has increased from zero just two years ago to about ten this year, with more anticipated following the publication of all our services in the NBS calibration service manua 1, SP-250, and appropriate publicity releases in journals and technical magazines. If we are indeed in this transition region, our own customer lists will not be significant in evaluating the validity of our conception of the National Measurement System until we have undertaken a thorough communication, including workshops and seminars, with the many consumer groups identified in section 3.1.1. 
Table 6. List of customers requiring NBS ultraviolet radiometric services 1972-1976

Astrophysics

University of Hawaii

Kitt Peak Observatory

Aeronomy, Atmospheric Sciences

Air Force Cambridge Research Laboratories

Aerospace Corporation

Johns Hopkins University

Massachusetts Institute of Technology

Naval Research Laboratory

University of California (Berkeley)

NASA Goddard Space Flight Center

Max-Planck Institut (Germany)

\section{Basic Research}

National Bureau of Standards

University of Florida

University of Western Kentucky

Technical University of Wroclaw (Poland)

University of Belgrade (Yugoslavia)

Centre Etude Atome (CEA, France)

NASA Langley Research Center

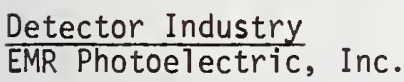

Fusion Research

Gulf General Atomic

Lamp Industry

Sylvania

North American Philips Corp.
Planetary Sciences

University of Colorado

Johns Hopkins University

Laboratory for Atmospheric and Space Physics

Regulatory Agencies

National Institute for Occupational

Safety and Health

Bureau of Radiological Health

Solar Physics

Naval Research Laboratory

NASA Goddard Space Flight Center

Ba11 Brothers Corporation

Harvard College Observatory

Air Force Cambridge Research Laboratories

Centre National de la Recherche Scientifique (France)

Standards Laboratories

Stanford University

Max-Planck Institut (Germany)

Optronics Laboratories, Inc.

EMR Photoelectric, Inc.

NASA Goddard Space Flight Center

National Physical Laboratory

(England)
Almost hidden in this table are several high leverage customers: the $1 \mathrm{amp}$ and detector industry and other standards laboratories. These are discussed more in the next section.

\subsubsection{Alternate Sources}

In the introduction it was mentioned that the Bureau's aim is not necessarily to perform all the many calibrations required by government and industry, but rather to undertake those calibrations and other services that will make the National Measurement System function most effectively and economically. Unfortunately, decisions which are meant to determine where we can obtain maximum leverage from our programs are difficult to make and often require tedious technology assessments. However, these decisions as weil as the social and economic impact analysis are sometimes made for us if we can only recognize it. For example, when we see a major lamp or detector manufacturer set up and advertise a calibration service at their own expense, it can be assumed that there is a serious need in the measurement sector and that there is money to be made. At that stage, NBS should be prepared to support these programs as much as possible and provide a calibration baseline which would lend more credence to the claims of the manufacturer and probably result in increased sales and applications.

The above scenario may seem very obvious and logical but, practically speaking, this is what can and sometimes does happen: NBS may not instigate a contact ("They know we are here--if they need us, they'11 call us"); industry may not instigate a contact ("I've never heard of any NBS work in this area" or "I don't think they are set up to do such calibrations, but if they are, we'll probably hear from them"). Non-communication!

In this microstudy we have seen interest expressed by the instrumentation industry not only by words but by action.

"We urgently need a standard uv source (or 
detector) to provide us with an absolute calibration. We supply, with each of our detectors, an individually measured absolute spectral response calibration....

Presently we cannot maintain our absolute uv calibration accuracy to the accuracy which we desire.... We would like to cooperate with NBS in any way possible on this problem."

NBS must be sensitive to situations like this and must be more responsive.

\subsubsection{Funding Sources for NBS Services}

The radiometric calibration programs both in the Far UV Physics Section and the Plasma Spectroscopy Section were initially funded almost entirely by National Aeronautics and Space Administration outside contracts. Energy Research and Development Administration funding (fusion program) has now been obtained and essentially replaces the reduction in NASA funding which followed the termination of the Skylab program. In addition, there has always been miscellaneous funding from calibration fees. Such complete dependence on other agency funds makes it difficult, if not unethical, to reprogram or to change priorities in order to respond to other measurement sectors. What interaction has taken place with users outside the space and thermonuclear fusion community has so far been restricted to applications which have the same requirements. In order to be able to respond effectively and in a positive way (rather than with the attitude: "Here is what we have--do the best you can") to the National Measurement System, direct NBS funding is absolutely necessary.

\subsubsection{Mechanism for Supplying Services}

In the field of ultraviolet radiometry, NBS provides services to the National Measurement System in the form of: (1) instrument calibration; (2) use of NBS facilities for systems calibrations; (3) publications and talks; (4) committee membership; and (5) workshops.

(1) The instrument calibration services have already been described in section 4.2.1. Source and detector calibrations are done either through outside contracts, for example with the National Aeronautics and Space Administration or with the National Institute for Occupational Safety and Health, or under the auspices of the NBS Office of Measurement Services (200.00). Over the last five years, over 100 devices have been calibrated.

(2) Users have also come to NBS, for example to use the synchrotron ultraviolet source. This is done when the calibration requirements are such that either no transfer standards are available or the highest possible accuracy is desirable. The hydrogen wall-stabilized arc primary source has been used likewise [31].

(3) A list of publications and talks concerning far ultraviolet radiometry are contained in appendix D. In addition, the Data Center for Atomic Transition Probabilities has sold a total of 4000 copies of its critically evaluated data compilation and over 6000 copies of its bibliography surveys.

(4) One of the staff has served on the 1974 Atomic Energy Commission (now Energy Research and Development Administration) research panel on atomic, molecular, and nuclear data needs for controlled thermonuclear research. In addition, he is serving on an ad hoc committee studying the feasibility of a national fusion facility. Another of the staff acted as the chairman of the Apollo Telescope Mount (ATM) Collaborative Task Group on Radiometric Calibrations during the National Aeronautics and Space Administration Skylab missions.

(5) Several workshops have been organized by NBS staff concerning ultraviolet radiometric calibrations. Four workshops (1971, 1972, 1974 and 1976) were held primarily for space scientists and those instrument manufacturers who were involved in the space program. About 40 professionals from the U.S. and abroad attended each meeting. Similar but less formal workshops were also arranged in 1972 and 1973 in order to initiate a dialogue with the National Institute for Occupational Safety and Health and with the Bureau of Radiological Health. About 20 people attended each meeting. Finally, a workshop was organized in 1975 to discuss data and diagnostic needs of the controlled thermonuclear fusion measurement sector. About 40 leading scientists were invited and participated in this meeting held at NBS.

\subsection{Impact of NBS Services}

\subsubsection{Economic Impact of Major User Classes}

For purposes of an economic overview, the major user classes described in section 3.1 and 1 isted in table 4 can be grouped into the following general categories: (1) medical; (2) energy; (3) environmental; (4) space; (5) photochemical; and (6) instrumentation. NBS does not presently serve a 11 these areas, but should and probably will in the years to come. Therefore, the following discussion is relevant, at least as applied to potential users and potential NBS impact.

(1) Medical. We have seen that ultraviolet radiation is being used increasingly 
as a clinical tool: (a) in the treatment of several disfiguring skin diseases; (b) in a treatment for the prevention of tooth decay; (c) for bacteriological control in hospitals, schools, and industry; and (d) for the treatment of calcium deficiencies. In situations like these, the economic impact is coupled with the social impact and is almost impossible to calculate. For example, psoriasis alone afflicts nearly eight million Americans. What is the economic impact of treating these people? Surely it is far more than just the volume of ultraviolet lamps sold for that purpose. Freed from their affliction, major personality changes could be anticipated in the patients "who suffer the heartbreak of psoriasis" along with a corresponding improvement in their effectiveness as a contributing member of our society. What is the economic impact of possible el imination of caries (tooth decay) through large scale use of the protective polymeric coating which can be applied to the teeth with the use of ultraviolet radiation curing? Surely it is far more than the first order impact, the sales volume of the ultraviolet lamp system and accessories. It reduces our time spent in the dentist's office, thus increasing our own productivity. It also liberates the dentist from much routine work, thus allowing him more opportunity for specialized work like preventive dentistry, orthodontics, periodontics, and endodontics. The socioeconomic impact of medical applications is very large, but where does NBS fit into this picture?

It has been discussed in preceding sections how excessive ultraviolet radiation can be a hazard. Several regulating agencies have been established to oversee the use and applications of ultraviolet radiation. Currently, they find it extremely difficult even to specify hazard levels

since experiments, designed to determine such levels, in many cases have been 111 -defined due in large part to lack of adequate standards. Of course, once the limits are specified, the agencies must also enforce the regulations, but currently they do not have adequate standards on which to base their measurements.

Besides providing a base from which the regulatory agencies can operate, NBS has a direct impact in the actual medical applications. Ultraviolet radiation measurements need to be made. When they are made indiscriminately, misinformation is propagated. For example, in the cited dental application $(3.1 .1 .1(2))$ the manufacturer's recommended procedure is to first irradiate a sample of the liquid formulation prepared on a glass slide and "see how long it takes to harden".
This is an uncertain empirical approach and, having seen a dental hygienist carry out these instructions, we can safely predict that varying degrees of success or failure will be experienced. Too little exposure means that the formulation does not polymerize and will wash out; too much exposure can cause the coating to become brittle, crack in time, and to become ineffective. Radiation damage to the surrounding gum tissue may also be a problem. Although my own dentist has one of the units, he does not use it for preventive dentistry, but rather only to overcoat gold fillings: "If it sticks, fine; if it doesn't, no harm done." Personally, this dentist considers the unit a waste of his money. But the story does not end here. In 1975, the Bureau of Radiological Health ordered the manufacturer of this ul traviolet radiation appliance to tell its customers (about 20,000 dentists according to a company spokesman) to stop using the device until modifications were made to prevent incidents where leaking radiation has injured both dentists and patients [109].

The above sequence of events is an example of what can happen to new techniques when they are not accompanied with adequate measurement capabilities: lack of confidence in empirical application procedures, inconsistent results, lack of enthusiasm, diminished sales volume, and finally the product is no longer available. Similar cases for the role of basic standards can be made for other examples. In addition, biomedical measurement problems are often compounded by the fact that radiation of one wavelength can influence the effects caused by another wavelength (see the discussion on the treatment of jaundice in section $3.1 .1 .4)$. Without adequate standards one can go in circles for years; with standards real scientific progress followed by effective commercialization can take place.

(2) Energy. An ultraviolet radiation measurement system is useful in fusion system research and development, solar energy systems analysis, and in conservation programs involving the illumination efficiencies of lamps. All of these areas have obvious economic value and have been discussed in detail along with appropriate documentation in previous sections.

In fusion systems the energetic ultraviolet radiation emitted by contaminants is a major energy loss mechanism and is currently limiting progress. Scaling calculations which depend eriticalzy on accurate measurements are currently being made to estimate the practicality of larger, extremely expensive devices.

The operation and lifetime of solar energy systems are affected by radiation damage to 
the materials. Accelerated weathering machines or solar simulators are used to estimate the various effects of prolonged exposure to the sun. Again, the reliability of such scaling depends criticalzy on accurate measurements. In addition such tests are complicated by the fact that certain effects caused by exposure at one wavelength may sometimes be changed or even neutralized when the sample is subjected to radiation at another wavelength.

The use of arc lamps for illumination purposes is extensive mainly because the lamps are typically three or four times more efficient than incandescent light sources. Because the economic impact is so large, competition is keen. In this case, the manufacturer is interested in minimizing ultraviolet radiation and maximizing the visible radiation from the lamp. The company that can demonstrate that it can provide a slightly more efficient lamp usually gets the coritract. U1traviolet radiation standards stimulate progress in this area and therefore impact on the international balance of trade and the overall cost of illumination.

(3) Environmental. The earth is protected from deadly solar ultraviolet radiation by a thin layer of stratospheric ozone. Any change in the density or thickness of this absorbing layer can have disastrous consequences. The results of a study by the National Academy of Science and Engineering indicated that even a 5 percent decrease in the ozone concentration would lead to 8000 additional cases of skin cancer. They were not able to calculate due to lack of data, but estimated that there would be even more serious effects on. important agricultural plants and ocean plankton which are so necessary in the ecological balance of our breathing air. What can cause a change in the ozone concentration? Combustion products of high flying airplanes (supersonic transport fleet), the freon propellant in common aerosol products, and perhaps even industrial smog--all have been shown to have such an effect. The board recommended the establishment of worldwide monitoring stations to detect small changes in solar ultraviolet radiation transmission. Where does NBS have its impact? First of al1, high accuracy standards are needed in order that all stations have a uniform base of reference. Otherwise, measurements in one place cannot be compared to measurements in another place, the significance of any one measurement is diminished, and global patterns are impossible to determine. Secondly, standards are needed because, practically speaking, systematic trends are what are most interesting, and the identification and quantification of trends in a reasonable amount of time require very high measurement accuracy. What is the economic impact? Again, it is far more than measurement equipment sales volume--such measurements could alert us to an ecological effect of worldwide proportions and give us time to modify the conditions which are responsible for changes in the ozone layer.

(4) Space. The United States still maintains a large national program in the space sciences. Almost one billion dollars a year is being spent on the development of the space shuttle. Routine shuttle flights in the 1980's are expected to cost 100 thousand dollars per hour of flight. Many millions of dollars are being expended annually for rocket and satellite experiments. Often the key components in these experiments are ultraviolet radiation measurements which cannot be done on the earth because of atmospheric absorption. Government, universities, and industry are presently involved; with the space shuttle, the involvement is expected to be even greater since flights will be less expensive and more convenient, and the weight and power specifications on payloads will be less restrictive. The National Aeronautics and Space Administration has demonstrated its continued interest in basic standards by actively supporting the NBS ultraviolet radiometry program. In an area where capital investment is very large and where the scientific output depends critically on standards, NBS participation should be considered as a high leverage impact.

(5) Photochemical. Every one of the photochemical applications described in section 3.1.1.1 was industry-oriented. Some of them have tremendous economic impact. For example, the use of radiation curing in the textile industry requires from 10 to 50 percent less energy than is required for a solvent-saturated heat cure which is among the largest energy consuming operations in textile mill processes. In addition, radiation curing is a non-polluting process [39, $41]$. The use of ultraviolet radiation in the wood finishing industry is also on the increase; one industrial representative estimates that as much as 20 percent of a 11 industrial finishes may be cured by some form of radiation by the end of the decade. The role of radiometric standards is as follows:

(a) New techniques require accurate documentation for the process to gain widespread commercial acceptance;

(b) Commercially available products need standards in order to meet specifications and to compete in a national and international market place; and

(c) The user needs standards in order to 
check specifications, to maintain quality control, and to monitor health and safety of workers.

As an example of these impacts, consider an industrial assembly line process in which certain manufactured items like paper products, glass, metal parts, microcircuits, or textiles are being coated by radiation curing. (a) For the technique to be accepted in the first place, it would have to be well understood so that performance could be guaranteed. (b) The equipment which would enable the assembly line to operate at the highest speed is the most desirable (maximum ultraviolet flux in the most effective bandpass). (c) Periodic calibrations in the field are necessary to guard against aging of the equipment due to radiation damage of the optical components.

(6) Instrumentation. The economic impact of this group has been discussed in detail in sections $3.1 .2(3)$ and 2.2.2.2. The annual value of shipments of a variety of ultraviolet emitting lamps and fixtures doubled between 1958 and 1963 and almost doubled again to the 1967 figure of 140 million dollars, according to the U.S. Census of Manufacturers. The estimated U.S. market for uv-vis spectrophotometers is 100 million dollars annually. What is the impact of NBS? This was discussed in section 3.1.2(3), but the main reason that manufacturers need standards is to enable them to meet (and give) specifications, to substantiate claims against an ultraviolet emitting product, and to compete in an international market.

\subsubsection{Technological Impact of Services}

This subject has already been covered in the preceding section on the economic impact of major user classes. However, there is perhaps one technological area that was not relevant in the economic discussion but is relevant in a discussion of technological impact: exploratory research.

Several areas of research could have significant repercussions economically (and socially), especially far ultraviolet and $x$-ray lasers (section 3.1.1.7) and photobiology (section 3.1.1.8). Documentation from industry researchers supports the idea that whole new industries could be formed using intense ultraviolet laser sources for petrochemical manufacturing, drug and pharmaceutical synthesis, and isotope separation. $x$-ray lasers are a long way off, but their obvious usefulness in a clinical sense is presently serving as a stimulus for their development (see also section 3.2). New insights into the mechanism of biological structure and activity through the use of far ultraviolet absorption spectroscopy, sometimes in combination with other tech niques like liquid chromatography (section 3.2), are possible. Because of the enormous potential impact of these new areas of research, an ultraviolet standards program designed to respond to the developing needs and to provide essential services (including facility sharing as in the case of the NBS synchrotron radiation facility) is necessary.

\subsubsection{Payoff from Changes in NBS Services}

NBS is presently unable to serve most of the areas requiring ultraviolet radiometric standards. This is partly due to the fact that our program is relatively young and partly due to the fact that it is almost entirely supported by other agency funds.

What would happen if NBS services were terminated altogether? The answer is: both a primary and a secondary negative impact. The space science measurement sector and the radiation health and safety regulatory agencies would miss us the most and would have to independently develop their own internal calibration facilities or depend upon a secondary standards laboratory to do the same. These are not bad alternatives, especially if the radiometric requirements were very narrow and applied only to a certain sector. However, one of the findings of this study is that the user sector is quite broad and diversified, that a National Measurement System, although scattered and diffuse, does exist, and that, therefore, the National Bureau of Standards ought to be involved.

Besides this first order negative impact, a much larger secondary impact would follow. The previously discussed social and economic impacts of those areas requiring an ultraviolet radiation measurement capability are reaz. Without a centralized standards program at NBS, it is believed that many of these areas will drift, go in circles due to the propagation of misinformation, * and at best, evolve in a very uncertain and erratic way. We will not witness development--this depends upon learning from previous experiences and extending and applying techniques in different ways and directions; it implies order and knowledge. Rather we will witness what might be termed the invention of gadgets. The potential for growth will not be realized, and our society will not enjoy the economic

*This situation has been pointed out several times in this microstudy through statements by correspondents from various sectors of the National Measurement System. 
and social benefits which would follow from that growth.

What would happen if NBS services were maintained at the status quo? The answer is: only the secondary negative impact, as described above. NBS is presently unable to serve most of the important developing areas requiring ultraviolet radiometric standards. This is partly due to the fact that our involvement is relatively recent and partly due to the funding situation. Without a much stronger commitment in terms of manpower and facilities, NBS cannot interact in a positive way with the photochemical industry, the biomedical community, and all the other sectors whose needs have been identified and documented. At present, we are not even able to serve adequately the demands of the regulatory agencies.

What would happen if NBS services were intensified? The answer is: good things. These good things are what a large percentage of this report has been trying to define. High leverage areas could be impacted such as the photochemical industry, medical and therapeutic treatments, environmental lighting applications, the instrumentation industry, the environmental sciences, the energy community, photobiological and bacteriological applications, national health and safety, plasma chemistry, and, of course, space sciences. There are priorities among these areas, of course, but fortunately many of the requirements have similarities. The recommended expansion of NBS services is described in section 4.5 .

\subsection{Evaluation of NBS Program}

As described in section 4.3, the present NBS program was set up with National Aeronautics and Space Administration funds to solve space science calibration problems. Attempts to respond to other users' needs, or even to find out if there are other users, have been secondary to meeting National Aeronautics and Space Administration obligations. NBS is in the position of saying, "This is what we have, can you use it?" rather than "Tell us what you need and we'11 try to provide it!" Presently NBS does not play a pivotal or central role in the Far U1traviolet Radiometry portion of the National Measurement System. There is little dialogue with the System, and even within NBS the ultraviolet radiometry efforts, which are divided among several sections, suffer from a communications problem brought on partly by an instinct for survival in times of constant (if not diminished) funding.

\subsection{The Future}

The status and future trends of the System as a whole have been discussed in section 3.2. What about the future of NBS in this System? In our opinion, if NBS is to meet its responsibility as a key component of the ultraviolet radiometry National Measurement System as it is now evolving, it must maintain its present expertise in both the Plasma Spectroscopy and Far UV Physics Sections and consider the following immediate programs where it is deficient:

(1) Develop reliable transfer source standards (a) in the region $110 \mathrm{~nm}$ to $165 \mathrm{~nm}$, with uncertainties of $1 \%$ and (b) below 110 $\mathrm{nm}$, with uncertainties of $10 \%$ or better. (2) Develop a spectral irradiance source standard for the region $110 \mathrm{~nm}$ to $250 \mathrm{~nm}$, with uncertainties of $1 \%$.

(3) Develop both the hydrogen arc source and the NBS synchrotron (storage ring) as complementary sources of absolute spectral radiance.

(4) Extend the calibration range of standard detectors to fully cover the range 5-400 $\mathrm{nm}$, with uncertainties of $1 \%$.

(5) Develop a program which would result in an NBS service providing for monochromator or filter transmission calibrations in the far ultraviolet.

(6) Initiate a program where standards from the different sections within NBS can be cross-calibrated and compared with each other. Internal consistency should be the first thing we should check. This would include such independent standards as the NBS photodiodes (232.03), synchrotron radiation (232.03), hydrogen arc radiation $(232.07)$, electrically calibrated radiometers (232.14), and tungsten lamps (232.04).

(7) Initiate more workshops and contacts with the various interest groups similar to the successful meetings described previously which involved the health and safety regulatory agencies, the National Aeronautics and Space Administration, and fusion scientists. It should be clear from the lack of interaction between the standards community and the majority of users that NBS is not keeping up with the evolving National Measurement system. Photochemistry and biomedical applications should be the first fields where NBS should generate new initiatives.

(8) Initiate a formal measurement assurance program (MAP) for all ultraviolet radiation services--presently only informal arrangements exist. 


\section{SUMMARY AND CONCLUSIONS}

It would be a mistake to consider the ultraviolet as just the lower end of the visible wavelength range and to arrange priorities accordingly. Ultraviolet radiation as defined in this microstudy covers a rather wide range of the electromagnetic spectrum, and because of its special characteristic of being very energetic, the uses and applications are quite different than those for visible radiation. Ultraviolet radiation should be considered as a separate field from visible or optical radiation in the same way as radio waves and microwave radiation are considered distinct from visible radiation.

An ultraviolet radiation measurement system exists and is composed of central

standards authorities, documentary specification institutions, regulatory agencies, secondary standards laboratories, an instrumentation industry, and a diverse users

group. Commination among these sectors is ineffective due essertialiy to the lack of a comor. language--the language of acmrate measurements. The NBS has so far played a very minor part in the evolving measurement system and must bear part of the responsibility for this lack of dialogue.

The key findings of this microstudy can be summarized:

(1) The exploitation of the unique properties of ultraviolet radiation and the consequent evolution of a National Measurement System are relatively recent developments.

(2) There is a diverse users sector which has significant economic and social impact.

(3) There is very weak interaction among the different elements of the National Measurement System.

(4) Very little use has been made of the resources that could be available from secondary standards laboratories and the instrumentation industry.

(5) Despite the potential impact and leverage involved in supporting and contributing to the measurement system, the NBS participation has been indirect and inadequate. A positive, seven-point program which would greatly improve this situation has been outlined in the preceding section, 4.5.

(6) Demands and requirements for standards are rapidly increasing. These standards are essential for continued growth of those industries and technological areas which utilize and depend upon ultraviolet radiation measurements.

And firally, it should be obvious that we must preach the gospel, the "good news". We must tell people what we have and what we intend to do--not only those scientists whom we meet at our own topical conferences, but also those in other neighboring disciplines. It must be done in such a way that some feedback is possible so that we know whether or not we are serving the community in the best possible way. Invited papers, publications in trade journals, committee memberships, workshop participation--all are possible forums. However, without a strong commitment in terms of program support from NBS and the Department of Commerce, there will be no incentive and few will be able to justify the time to act on these possibilities. The NBS program will continue to be oriented toward outside contracts and to the specific missions of particular agencies or groups rather than to the many needs of the evolving far ultraviolet national measurement system. 


\section{APPENDIX A. METHODOLOGY OF THE STUDY}

The information sources used to determine what areas of technology are presently or potentially impacted by ultraviolet radiation calibrations were as follows:

(1) technical magazines

(2) customer lists,

(3) topical meetings,

(4) personal contact,

(5) unsolicited contacts, and

(6) data sources within NBS.

Technical magazines like Physics Today, Laser Focus, Industrial Research, Optical Spectra, and Electro-Optical Systems Design are a rich source of publicity releases on current state of the art technology. Generally the magazines are free and can be delivered monthly to one's office. Many initial contacts for this survey were made through this method and were followed up usually by letters and phone calls. Because of the convenience, staff members should be encouraged to monitor such magazines. If more people were to cut out and fize the articles of interest, the program analyst or technology forecaster who is faced with developing a realistic scenario would have immediately available from his organizational unit a solid source of base material.

Customer lists were a second source of user information. These were followed up as usual by letter contacts and in some cases phone calls. However, this method is complicated slightly by the fact that in many cases the original purchaser has relocated. One must decide then either to abandon the contact or to follow it up with additional efforts to determine the current users.

Topical meetings are obviously a good place to make contacts. However, there are many fields impacted by our services and, unfortunately, we are usually not aware of their society functions and meeting schedules. This was especially clear to us when the president of one of these "other" societies observed

"One of the subsections of our Society is

phototechnology. While I can appreciate

that the real technology of building your

lamps is more closely related to the

Optical Society, the users may belong to our Society."

Personal contact is probably the best way to convey information, especially if followed up with a confirming letter. We have. made a few such contacts as part of the National Measurement System study, notable among which were two NBS mini-workshops in which we met with representatives from the Bureau of Radiological Health in one case and from the National Institute for Occupational Safety and Health in the other case.
We also organized and participated in four workshops to discuss ultraviolet calibration needs and capabilities with representatives from the space science community. A similar workshop was set up at NBS with the fusion community to discuss basic data and diagnostic needs. More of the workshops should be encouraged, especially involving fields that we don't normally have too much contact. with, for example, ul traviolet lasers, photochemistry, and biomedical applications.

Unsolicited contacts were quite frequent in this survey, mainly because of our listing in the NBS Index of Technical Activities under the general subject, Ultraviolet Radiometry. Most of the time the requests are minor and can easily be handled. For example, we had the opportunity to advise the White House concerning the selection of window pane material which would have low ultraviolet transmission and thus would minimize the fading of the Blue Room carpet and curtains; a similar request from the National Gallery of Art was also received and answered. On the other hand, our initial contact with the National Institute for Occupational Safety and Health was also made in this way and culminated in the formation of a cooperative ultraviolet calibrations program with NBS.

All of the above can be considered data sources outside of NBS. Within NBS, there are basically three independent sources of National Measurement System data: the Plasma Spectroscopy Section, 232.07, the Far UV Physics Section, 232.03, and the Optical Radiation Section, 232.04. Besides depending on individual contributions from members of each section, we have made great use of issue studies that have been developed by each of these sections over the last three years. However, although there is much in common, communication and cooperation between the three sections could be improved. For example, there have been occasions when a request for calibration information or a particular service has reached one section and goes essentially unanswered because the expertise, unbeknownst to the person involved, lies in one of the other sections.

What about the significance of our sampling? Customer lists ought to be solicited from the various ultraviolet lamp and detector manufacturers, for example those discussed in section 2.2.2.2. These lists could then be compared with the users described in section 3 . Such a survey ought to serve as an internal check on the significance of the applications and may lead to some modifications on our conception of where the main activity in the ultraviolet radiation National Measurement System is centered. 


\author{
BLACKBODY SOURCES \\ AGA Cor \\ Advanced Kinelics Ine \\ Aesulux Lighit Co Inc \\ OBA SYstems/Electro Dptics Oiv pg 36 \\ Eleciro Outical Industies Inc \\ The Eupley Laboraloiy \\ Gilol Associales Inc \\ Inlared liddusiries Inc \\ licon Inc \\ Land Instlunients Inc \\ INFRARED SDURCES \\ AGA CoIp \\ Advanced Kinlencs lic \\ Aerolux Lighi Co Inic \\ Chicagu Minialure Lanen Woiks \\ Chissire Elecinic \\ DBA Systems/Electro Dptics Div pg 36
The Ealing Coip/Optics Drv cover 2 \\ Elect10. Oplical Indusitues Ine \\ The Epuley Labolalor \\ CTE Sylvania Inc \\ Gamma Scientific inc pg 37 \\ Gerielal Electuc Min Lanip Piod \\ Greol Associares Inc \\ Panovia Divicaniad Piecision \\ Hainck Scienuile Corp \\ Holobedm Inc \\ Illunination Industues Inc \\ Kull Engineeung Ine \\ Kull Engineeling Inc
Land Instiumenis Inc \\ MERET line \\ Monsanio/Eleci Special Pidis \\ Oplical Associales Inc \\ Oplical Communications tnc \\ Opilition Inc \\ Oplion lic \\ Photobell Co Ine \\ HCA \\ Ragnk Precision Induslues Inc \\ Sanders Assoc Inc/E 0 Dir pg 7 \\ Sania Haibala Research Cenier \\ Sensol Technology Ine \\ Shiugoio Indusiries Lid \\ Syeclionics thic \\ Wainecke Eleciion Tubes Inc \\ Westinghiouse Eleciuc Coip
}

LAMPS, Cold Cathode, Dark Trace

Aerolux Lighr Co Inc

Opucal lndusures ine

Shigolo induslities Lid

LAMPS, Alkali Vapo

Hamanalsu Corp

Holobeam Inc

ILC Technology

Klinger Scientific Appaatus pg 42

Micther son Instiument Corp

Wainecke Eleclion Tubes Inc

LAMPS. Argon

Aerolus Light Co ine

Chicago Minialule Lamp Woiks

EGå G Inc/Electro-Dptics Div cover 4

The Ealing Corp/Optics Div cover 2

Edmund Scientilic Co

General Flecinc Min Lamip Prod

IIC Technology

Klinger Scientific Apparatus po 42

McPherson Insli umeni Corp

Shigolo Indusiues Lid

Uhia Violel Producis Inc
Wesinghouse Electic CoIp

LAMPS. Flash

Amenican Uliraviolel Co

Amglo Coip

Aissio Gird Lamp Pioducis Inc

Beikey Technic

EG\&G Inc/Electro-0ptics Div cover 4

Glass Instl uments Inc

ILC Technology

IIluminatuon Indusulues Inc

Kemline Laboraloues Inc
Oplition linc

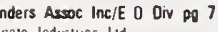

Shigoto Indusilies Lid

Siemens Colp

u S Scientilic Insinumenis Inc

Vollaic Tubes Inc

West Coasi Research Coup

Westinghouse Elecilic Coip

Xenon Corp

LAMPS. Hollow Cathode

Aeroitux Light Co Inc

Bausch\&LLonib Arialyucal Syslenis

The Ealing Corp/Dptics Div cover 2

Eisher Screntulic Co

Hanlanialsu Coup

Oplical indusines Ine

Ralik Piecision Industues the

Scientilic Selvices Co

Speciro Pioducis Inc

LAMPS, Helium

Aerolux Lıghı Co Inc

Alisio Gird Lame Pioducis in

Black Lighı Easteı

Chicago Minalute Lamp Woiks

The Ealing Corp/Optics Div covel 2

Edinund Scientilic co

George W Gales \& Co Inc

J A Oplic:

Klinger Scientific Appaıatus op 42

McPheison Insirument Corp

Opical Indusilies Inc

Shigolo Induslives tid

Surlace Finishes inc

LAMPS, Incandescent

Aerolux tighı co Inc

B \& E Enieipi|ses

Chicago Minializle Lamp Woiks

The Ealing Corp/Dptics Div covel 2

Edmund Scientilic $\mathrm{Ca}_{0}$

Cieorne W Gates \& Co Inc

Geneial Elecilic Min Lamp Piod

Gilway Sales Co

Klinger Scientific Apparatus po 42

Lumitron Co

Macbeth Sales Corp

$\mathrm{J}$ A Noll CO

Pinlies Div ol Relac

Piecision Lamp Inc

Shelly Associales

Shigolo Indusiries tid

Stan A Matic Corp

Svivania Minialure Lghing Pids

Technical Insliument Co

Wagreel Elec Corp/Tung Sol Oiv

Westinghouse Eleciıc Curp

LAMPS. Infrared

Aerolux Lighı Co Inc

Chicago Minaluie Lamp Woiks

Chussie Elecluic

EG\&G Inc/Electro-Optics Div corver

The Ealing Corp/Optics Div cover 2

Edmund Scienulic Co

CTE Sylvania Inc

General Eleciuc Min Lamp Prod

Hanovia Oly/Caniad Piecision

Holobeanl In

Illumination Indusures inc

Monsanto/Eleci Special Pidis

Optical Indusilies Inc

Duallz Radiation Colp

Shigoto Indusilies tid

Skàn A Malic Coip

Watnecke Elecison Tubes Inc

Westinghouse Flecliic Corp

LAMPS. Krypton

Aesolux Lighi co Inc

EG\&G Inc/Electro-Dptics Div cover 4

The Ealing Coip/Optics Div corvel 2

Edmurid Scientilic Co

Hadron Inc Koiad Depi

ILC Technology

Klinger Scientific Appaıatus po 42
Oplical Industries Inc

Ulira Violel Producis Inc

Westinghouse Elecilic Corp

LAMPS. Mercury. Metal Additives

Advanced Kmences Inc

Ameucan Uituaviulet Co

Beikey Technical

Gaeunner Scienulic Coup

Ceorge W Gates \& $C_{0}$ Inc

Hanovia Div/Canirad Piecision

ILC Techulogy

Illunination lidustries inc

Klinger Scientific Apparatus pg 42

Uptical industies Inc

Ouarte Radiation Coip

Wha Violes Producis Ine

Westinghouse Electic Coip

LAMPS. Meıcury-Xenon

American Uiltiavulet Co

Berkey Technical

Chussie Elecl

PIC Inc

The Ealing Corp/Optics Div cover 2

Hanovia Div/Caniad Piecision

ILC Technology

Illumination Industries Inc

Klingar Scientific Apparans pg 42

McPherson insitument Colp

Oplical Industires inc

Direl Coip of America po 2

Schoetfel insirument Coip

Shigoro Indusines lid

Ulira.Violel Pioducis Inc

Wesinghouse Elecilic Coip

Xenon Coip

LAMPS. Miniature

Aisto Gird Lamip Pioducis Inc

B \& F Enterprises

Chicago Minaluie Lanip Woiks

Dialught Coip

Eder Instiument co Inc

Edniund Scienulic Co

Ceoige $W$ Cales 2 co Inc

Geneial Eleciric Min Lamp Piod

Gllway Sales Co

J A Noll Co

Opicical Indususues ine

Ppitcal Indusinles Inc

Pinlites Div of Rel

Precision Lamip line

Sigrialıe Dv.General Insiı Co

Skan. A Mauc Coip

Sylvania Minaluie Lghing Pids

Ulisa Violer Products Inc

Weich Allyn loc

Westinghouse Electric Coip

LAMPS, Point Soutce

Ace Scientific Supply Co Inc

Beikey Technical

EG\&G Inc/Electro-Optics Div cover 4

Edmund Scientilic Co

Gaeriner Scienilic Corp

George $W$ Cares \& Co inc

Gilway Sales Co

ILC Technolog

IIT Eleccisen Tube Ory

Optical Indusilies Inc

Duarle Radialion Coip

Schoellel Insirubient Co

Shigoto Induslines tid

U) S Scieutitic Instiuments Int

Wesinghouse Elecilic Coip

Xenon Coup

LAMPS. Shor AIC

American Ulitravioler Co

B \& F Enleipuses

Beikey Technical
Chistie Eleclicic

EG\&G Inc/Electro-Optics Div covel 4

The Ealing Coup/Dptics Div cover 2

Cearge W Gates \& Co Inc

Hanuvia Divi/Caniad Piecison

ILC Technology

Eleclion Tube Div

Klinger Scientific Apparatus pg 42

Opical Industues Inc

Dilel Coip of America po 2

Schaelle! Instiument Coip

Shigolo Industiles LI

Wesinghouse Flecinc oils Ine

LAMPS, Spectial

Ace Scientilic Supply Co lut

Berkey Techincal

Black Light Eastein

The Ealing Coip/Optics Dir cover

Caentnel Scientilic Coolp

Klinger Scientilic Apparatus pg $\mathbf{4 2}$

Optical Industries line

Oriel Corp of America og 2

Rank Piecisiun Industives lite

Schoeflel Insilunienı Colp

Scientilic Seivices Cu

Sloan Technology Corp

AMPS, Strobe

Aniglo Corp

EG\&G Inc/Electro-Optics Div cove

Edmund Scientitic Co

General Electıc Min Lamip Plod

General Radio Co

ILC Technology

Iilluirnation industues Inc

Kemline Laboratures linc

U S Scientific Instiultienis luc

Xenan Corp

\section{LAMPS. Tungsten-Halogen}

American Ultraviolet Co

Gauschis Loint Altalyucal SysteiI!

Berkey Technical

Coherent Radialion

Corion Instrunielli Coop

The Ealing Corp/Optics Div covel 
Westinghouse flectic Coip

\section{LAMP. Xenon}

Ameucan Uliraviole Co

Alssto Gind Lamp Pioducts Ine

Allas Elecuic Devices Co

Bausch\&lonib Analyutcal Sysienis

Beikey Technica

Candela Corp

EGSG Inc/Electro-0ptics Oiv cover 4

EGaG inc/E

EMR Photoelectiric

The Enling Corp/Optics Oiv cover

George W Gales \& Co inc

Haniamatsu Coip

Hanovia Oiv/Caniad Precision

Hughes Allcratt $C_{0}$

ILC Technology

IIT Election Tube Div

Illumination Indusiries Inc

Klinger Scientific Apparotus po 42

McPherson Instument Corp

Optical Industies Inc

Opical Radiation Coı

Oriel Colp of America pe 2

Phase $\cdot R$ Coip

Ouali Radiation Corp

Senders Aesoc Inc/E 0 Oiv pg $?$

Schoellel Insitunient Coip

Shigoto Industiles Lid

Sieniens Corp

U S Scientilic Instiunients Inc

Ultia-Violet Products Ine

Vollarc Tubes Inc

West Coast Research Corp

Westinghouse Electuc Coip

Xenon Coris

LEOS. Mear If (noneoherent)

B \& F Enterpuses

Cenir alab Semiconducio

Cłanex Corp

Fanchuld Caniera \& Instlument

Fauchild Senuconducto

Geneial Electirc Min Lamp Piod

Geneıal Sensors

Hewlelr-Packard

Lasel Olode Labolalories inc

Litionia

MEREI InC

Monsantu/Elect Special Pidts

Motolola Semiconductors Inc

Nippon Electuic Co.NEC Amenica

Option lne

Ouanium Sensing Inc

RCA

Sensol Technology Ine

Skan.A.Matic Coip

Spectionics Inc

LEO'S. Visiblo

B \& F Enterpuses

Calveil Elecifonics Inc

Chicago Miniatule Lamp Wouks

Oialight Coip

Fauchild Camera \& Instument

Fartchild Semiconducto

eulanu Eleculic Inc

General Elecuic Min Lamp Piod

Hewiell. Packard

Inflared Indusitues loc

Lillonix

Monsanio/Elect Special Prdis

Motorola Semiconduciors Inc

Nippon Electuic Co.NEC Ameuica

RCA

Shigoio Industiles Lid

Siemens Coip
Sola Sysiems Inc

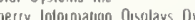

Wesunghouse Eleciric Coip

LEO ARRAYS. Nonimaging

B \& $\mathrm{f}$ Entergrises

Chicago Miniature Lamp Woiks

Fauchuld Semiconducio

Fertanti Electuic Inc

Goefz-Inland Sysiems ON

Laser Olode Labolaroiles Inc

LIII ONI*

MERET Inc

Monsanio/Eleci Special Pidts

Moloola Semiconduttors Inc

Oplion Ine

Sensor Technology Inc

Shigoto Industlies LId

Spectıonics Ine

LIGHT SOURCES. Carton Asc

Klingar Scientific Apparatus of 42

UIGHT SOURCES, Colomotic Reference

Cauadian Reseaıch Instuure

EGoG Inc/Elactro-Optica Oiv cover 4

Edimund Scientilic Co

Gemma Scientific Inc po 37

Macheth Colol \& Pholomelly On

Macbeth Sales Coip

LIGHT SOURCES. Electroluminescemt

Hughes Aucialı co

Laser Olode Laboratolies Inc MERET Inc

Shigoro Industiles LId

Sigmation inc

Signalıe Ov. Geneıal Insu Co

kan-A.Malic Coup

LIGHT SOURCES, Fiber Optic

Amer Opucal Corp.Fiber Optics

Areopix Technology Corp

Bausch \& Lomb

Chustie Electrit

Oavidson Optronics Inc

Oolan Jenne

Oyonics Inc

The Ealing Corp/Optice Oiv cover 2

Edel Instiunieni Co Inc

Edmund Scientilic Co

Electio Fiberuptics OIv/Naliec

Inconil Inc

Keystone Opucal Fibers Inc

Lenox Inst co Inc

Pank Precision Indusiues Inc

Sensol Technology In

Shelly Associates

Shigolo Industlies Lid

Skan-A.Matic Coip

$3 \mathrm{M} \mathrm{CO}$

Welch Allyn Ine

LIGHT SOURCES. Multiple Poim

Aleopixx Technology Corp

Opical Associales Inc

LIGHT SOURCES. Photonotric Reforence

Canadian Reseaıch Insutute

EGSG Inc/Electro-Optics Oiv cover

Edmund Scientilic co

The Eppley Laboialouy

German Sciontific Ine on 37

Gilot Associales Inc

Hollman Engineering Coup
Lighung Engneers Inc

hulo Research

Schoeflel Instumnen coip

LIGHT SOUACES Poin

Areopix Technology Coip

Davidson Optionics Inc

Oyonics Inc

EGs Inc/Electro-0ptics Oiv cover

The Ealing Corp/Optice Oiv cover 2

Gaerneer Scientilic Corp

Gamm Scientific Ine of 37

George W Gales \& Co Inc

Hollman Engineeuning Cor

Illumination Industues Inc

Oplical Associales Inc.

Orial Corp of America og 2

Schoellel tonstiument Corp

U S Scientilic instiuments Inc

LIGHT SOURCES, Self-Luminascem

Shigoro Indusiries Lid

$3 \mathrm{M}$ Co

LIGHT SOURCES, Spectrally Selective

Edmund Scientilic Co

Optical Associales Inc

Driel Corp of Amarica po 2

United Detecmer Technology Ine po 1

LIGHT SOURCES. Strobe

Amglo Coip

Bertey Technical

Chadwick. Helmuth co Inc

GaG inc/Electro-Optics On cover 4

General Radio Co

ILC Technology

Siemenis Coop

U S Scienilic instiuments Inc

Wabash Instiument Coip

Xenon Lolp

LIGHT SOUACES. XonOn ArE

Aurencan Uimaviuter $\mathrm{Co}_{0}$

Aninglo Coip

Ailas Electiuc Oevices co

Beikey Technica

Chadwick-Helmuin Co Inc

Chusste Eleciri

EGaG Inc/Electro-0ptics Oiv cover

The Ealing Corp/Optics Oiv cover 2

George W Gates \& $C_{0}$ Inc

Hanovia Oiv/Camiad Piecision

Hollman Engmeerning Corp

Hotobeanı Inc

Hughes Auccalı Co

ILC Technology

III Elecilon Pube

Illumination. ludusluss

Klinger Sciemutite Apparatus po 42

Lenus Insi Lo Hit

Opuical lidustites luc

Ophical Hastits linc

Orial Corp of Anerica po 2

Oriall Corp of

Schoeflel Instiument Corp

s̀entens Coip

U S Scienulic Instiuments Inc

Voltaic Tubes Inc

Wesunghouse Electuc Coip

MONOCHROMATOAS (Light Source)

Ace Scientitic Sugply Co Inc

Advanced Kinelics Inc

Amencaul Insiu umeni Co

Bausch\&Llomb Analyical Systems

EPOI Insti umenI OIV

The Ealing Corp/Optics Oiv cover 2

Gaerine! Scienulic Corp

Ganme Sciontific Ine pg 37

Giol Associates Ific

Haluck Sciennilic Corp

Heaih/Schlumbergeı Sa Insis

Mckee Pedersen Insti unients

Oputitiou Ine

Oriol Corp of Americe po 2

Rank Precision Indusilles Ine

SMI Ine

Schoellel Instiumen Coip

Technical Insliument Co

United Oetector Technology Ine pg 1

Carl Zeass Inc

OPTICAL PARAMETAIC OSCILLATORS

Chromalix

Oavidson Detionics inc

Sigualite Ov.General Instı co

SEARCHLIGHTS

Brookstone $C_{0}$

ILC Technology

Opucal Radiaion Coup

Tamarack Scienulic Co Inc

SOLAR SIMULATOAS

Atlas Electric Oevices Co

Caison Astionomical Insilumnis

Electio-0pical Industries ine

Exolech Inc

High Vollage Engneering Coip

Hollman Engneering Coip

Hughes Aucialt Co

IL Technology

Orial Corp of America po 2

Schoellel Instiument Corp

Tamaıack Scientilic Co Inc

\section{ULTRAVIOLET SOURCES}

Amencan Ulitazvioler co Alisio Gild Lamp Products Inc Allas Elecuic Devices co

Beıkey Technical

Black Light Easıeın

Christie Eleciric

Coherent Radration

Corron Instiviment Corp

MR Pholoeleciri

The Ealing Corp/Optice Oin cover ?

edmunt Screntilic Co

Geoige W Gales \& Co In

Gelman Insirument co

Glass Insı uments inc

Hamamatsu Corp

Hanovia Oiv/Canrad Precision

ILC Technologr

Illymunation Indusilues inc

Laser Energy Inc

McPheison Insti ument Coip

Mckee.Pedersen Instiuments

Oplical Indusiues Inc

Oriel Corp of America po 2

PQLL Inte'nalional Inc

Polysciences Inc

Projecina USA

Schoellel Insirument Corp

Scienulic Services Co

Shannon Luminous Marerials Co

Ianıarack Scientilic Co inc

Ulira-Violer Pioducts Inc

Voliaic Tubes Inc

Wesinghouse Electuic Coup

Xenon Coup 


\section{IN OPTICS}

Acron Research Cuip

Anersil ine of 45

Apuled instivirients Ind

Bromier Research Cuid

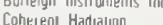

Conerent Hadratan

Conimerinal Oplical Cory

Cosno Opilics Inc

Oiveise Technologies Inc

The Ealing Corp/Optics Oiv cover

Edriahite Curp

Eicona Coin

Exolic Matellals Inc

FWW Indusilues

Raver Optical Assoc Ine

Gaesiner Scientulic loip

Grayco Oplical Cop

Groud 128 lac

Hantick Scienulic Corp

Heloolek/Optical Thu film On

Honewwell Test Insilumenis Dy

Inieınaiınal Lıgha Inc

liek Coip

Janos Oplical Core

Karr Lambrecht Corp pg 25

Kollsman Instrumen! $\mathrm{C}_{0}$

Lambda/Aintron Drvilnalo, Sy

Lase! Energy Inc

Lasel Oplucs Inc

Lenox Inst co Inc

McPherson Insicumenl Corp

Adoll Meller Co $_{0}$

Adoll Meller Co

Meriologic insilumenls Inc

Nye Opucal Co

Olympus Coip of America

Dimpus Coip of Amercis

Oplical Insiruments Caip

Oplical Producis Inc

Oplical Producis Inc

Oplucs Internalional

Opics Technology Inc

Pace 2

Pesic Qpical On/Bouns

E-O Div 20

Pholo-Oplix

Phorronics Corp

Precision Cells Ine

Precision Lapping as Do

Ovariz Pioducis Corp

Rolin Oplics Co

Fiank A Ruhmanis \& Co

SORL

SORL A Hih

Schoellel Insti

Special Dplics

Spectium Srstems Dir

leledyne Lamera Sysiems

Thermal Ameican Fused Ouari:

Tropel Jnic

Valpey Coip

Carl Zers Inc
IN TRANSMITTING MATERIALS (nOn-glass)

Acion Research Corp

Anersil Inc of 45

Atomergic Chemetals Co

Cryslal Sysiems Inc

Esce Pioducts

The Hasshaw Chemical Co

The Homalite Corp

Insaco Inc

Lambda/Aultion Oiv/Limon Sys

Merii Chemical $\mathrm{C}_{0}$

Nye Oplical Co

Ouariz Products Corp

WINDOWS. WV (non-glass)

Actun Reseacli Lump

Alherux tor

Ameisil Inc pg 45

Auplied instruments luc

Celatilatetal lisc

Counuenal Unical Cun

Conturenial Uplical Curn

Custllo Opilics ho

Diverse Technologies Inc

The Ealing Corp/Optics Div cover 2

Esco Pruduces

Esco Pruducls

Exoric Materials inc

Karl Feues Optical Assoc lac

Gaertiet Scieminlic Co

Glayco Oplical Corp

Harrick Scientilic Corp

The Hashaw Chemical co

Herron Opuical co

Hughes Aircialt $C_{0}$

IIC Technology

ITT Flectron Tube OIV

Insaco Inc

Janos Oplical Coro

Karl Lambrecht Corp po 25

Hugo P Keller Inc

Lanibda/Aitron Divilution S

Laser Energy Inc

Laser Oplucs Inc

McDonnell Douglas Electronics

McPherson Inssionieni Cory

Adoll Meller Co

Múlolento Optical Co Inc

Oprical Indusiries Inc

Perkin-Elmer Corp/E-0 Div po 20

Pheilo-Oplix

Photionics Coip

Quate Pidocis Corp

Duarli Pioducls Corp

Fiank A Ruhruamn \& C

Sciernitic Services Co

Space Optics General

Syecial Oplics

Specirum Sysieins Drv

eledvne Latrera Systenus

Theriulal Almerican fused Ouaris

Tyeo Suphisum OIv

valuey Curp 


\section{APPENDIX C \\ An International List of Companies Having an Interest in Plasma Chemistry Applications}

\section{BELGIUM}

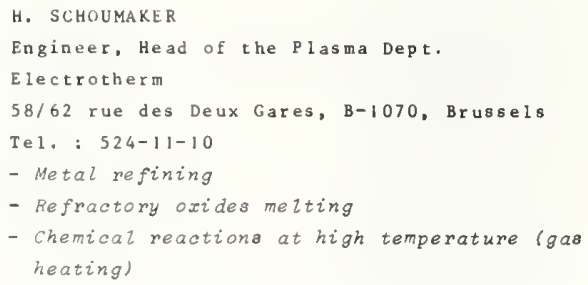

Dr. W. H. GAUVIN

Director of Research and Development

Dr. G.R. KUBANEK, Dr. R.J. MUNZ

Norands Resestch Centre

240 Hymus Boulevard, Pointe Clsire, Québec

Te1. : (314) 697-6640

- Industrial possibility of decomposing molybderite to yield molybdenum metal and elemental aulphur

- Application of plasma technology to the extracti of other refractory metala

Jesn-Paul R. HUNI Research Physicist Alcan Reduction Research snd Dev. P.O. Box 250, Arvida, Québec G7S $4 \mathrm{~K} 8$ TeI. : (418) 548-46!1 (3332)

Dr. Antoine THEORET Senior Scientist Institut de Recherche de l'Hydro-Québec Case Postale 1000, Varennes, Québec Tel. : (514) 652-8233

- Diajoncteura d huile, transformateura

- Tout procédé d'intérêt industriel (ex. : métalturgie extractive).

\section{FRANCE}

Pierre J. 80RTAUD

Directeur des Recherches Produits Electrofondus Sté Européenne des Produits Réfractaires 84130 Le Pontet Tel. : (90) 31-90-00

Fusion et travaiz des mélanges d'oxydes fondus

\section{André 8RUNET}

Ingënieur

Office National d'Etudes et Recherches Aéroapatisles

29, av, de ls division Leclerc, 92320 Chstillon

Te1. : 253-50-80

- Flowing plaamas of high denaity

- Simulation of ionoapheric plaama

- Hollow cathode arc discharge

M. CARON

Ingénieur à $1 \mathrm{~s}$ Direction Scientifique Pechiney Ugine Kuhlmann

23. rue Balzac, 75360 Psris Cedex 08

Te1. : 766-52-00

Dépota d'oxydea 
Jean Daniault

Ingénieur

Comissariat à l'Energie Atomique

C.E.N.G., B.P. ${ }^{\circ} 85$, Centre de Tri

38041 Grenoble Cedex

Te1. : 97-41-11

- Etude sur les caractéristiques des plasmas de projection. Etude systématique de revêtements. Applications en fabrication

- Ootention de couches minces par plasma inductif

Robert GUENOT

Coordination des Recherches.

Centre de Recherche Claude-Delorme, Société

L'Air Liquide

B.P. 126, 78350 Jouy en Jos as

Ie 1. : $956-80-20$

- Synthese par plasmas chauds et froids lexcitation selective pour synthesel

- Décharges et analyses chimiques

- Ozoneurs et gênérateurs d'orygène singulet

- Gaz actifs dans les plasmas de coupage des métaux

Jean-Pierre KIEHL

Directeur Centre de Recherches de Vénissieux Sociétê Européenne des Produits Réfractaires 10, rue de 1'Industrie, 69631 Vénissieux

Te 1. : Lyon 74-20-81

Projection ae pouires céramiques, notamment

c'alumine pour la réalisation de certaines

études de réfractaires

MOTtOO PERLE

Chef de Groupe Recherche Soudage

Centre de Recherche Claude Delorme, L'Air li-

quide

B.P. 126, 78350 Jouy en Jos as

Te 1. : $956-80-20$

Soudage, Coupage

Joseph RECASENS

Centre de Recherches, Sociêté Europêenne de

Produits Réfractaires

84130 Le Pontet

Te 1. : (90) 31-90-00

Helting, Welding, cutting, coating

Georges SAYEGH

Responsable du Département d'Etudes Scientifiques

Sciaky S.A.

119, quai Jules Guesde, 94400 Vitry

Te 1. : 680-85-D7

Utizisation du plasma dans le soudage des métaux

M. TRE VEDY

Ingênieur à l'I.R.S.I.D.

57210 Maizières les Metz

Te1. : (87) 60-21-54

Four d are

\section{GERMANY}

Dr. G. FRANCK

OSRAM Research

8 Münich - 90, Hellabrunner-Str. I

Tel. : $089 / 62133321$

- Ion-molecule reactions

- Associative ionisation

- Mass spectrometry of Plasmas

Dr. Kar 1 GEIERSBERGER

Direktor; Leiter Abt. Forschung und Entwicklung

Chemische Fabrik Kalk G.m.b.H.

BRD-5000 Köln 91, Kalker Bauptstr.22

Te1. : Kö 8296-330

Dr. Ludwig REHDER

Group Leader

Philips Forschungstab., G.m.b.h. Aachen

51, Aachen

Te1. : 024162071

- Light sources

- Atomic and molecular radiation, thermodynamico of plasmas, non equilibrium phenomena, elementary processes

\section{JAPON}

taruhiko $A B E$

Research $S t$ af $f$

Central Researchlab. Mitsubishi Electric Corp.

Minarishimizu, Amagasaki, Hyogo

Te1. : (06) $491-8021$

- Semiconductor integrated Circuits

- Display (plasma display)

- Breaker

- Ozonizer

- Syntheris

Yoshito FUKUBE

Senior Engineer

Institute for Super Materials, Ulvac Corp.

523 Yokota Sanbu-cho Sanbu-Gun,

289-12 Chiba Prefecture

Te1. : 04758 (9) 0131

- Melting of refractory materials

- Evaporation of refractory materiala

- Chemical reactiono 


\section{UNITED KINGDOM}

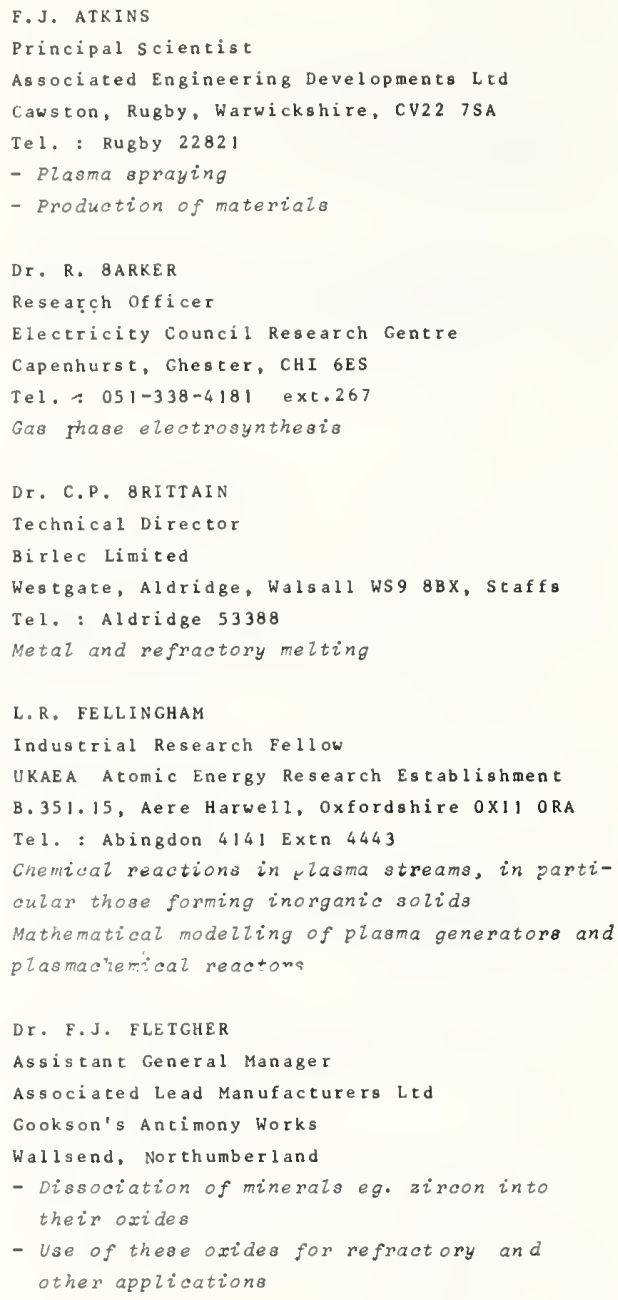

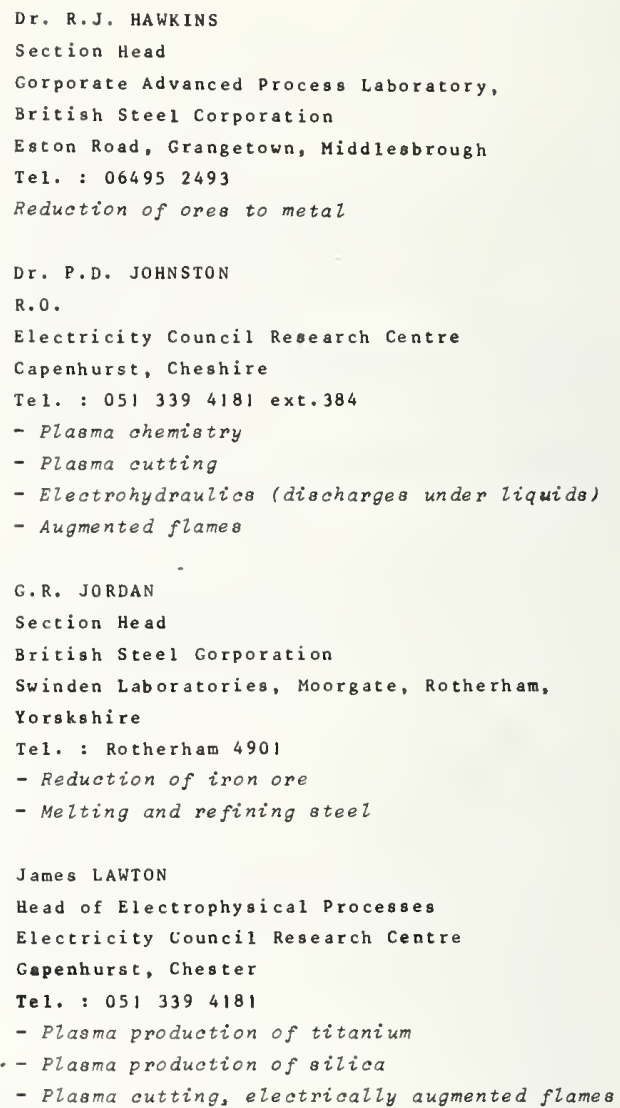


J.K. IYLKD

Research Director

Tetronics Research and Development Co.Ltd.

Faringdon, Dxon

Tel. : 0367-20224

- Extractive metallurgy at various levele up to.

final metal treatment.

- specialised refractories

- Occasional studies of inorganic and organic

endothermic syntheses

A.J. WICKENS

Group Leader (chemical research)

Borax Consolidated Ltd.

Cox Lane, Chessington Surrey

I el. : 01-397-6262

- Production of ultra-fine, high purity refrac-

tory powders.

John A. WINTERBURN

Research Manager

Thermal Syndicate Ltd.

Wallsend, Iyne and Wear

Tel. : 0632-625311

- Production of hydroxyl free vitreous silica.

UNITED STATES,

A.C. ADAMS

M.T.S.

Bell Telephone Laboratories

lurray Hill, N.J. 07974

Tel. : 201-582-3459

- Plasma deposition of thin films $\mathrm{SSi}_{3} \mathrm{~N}_{4}, \mathrm{SiO}_{2}$,

Si, $\mathrm{Al}_{2} \mathrm{O}_{3}$, SiC, BN) for Bemiconductors

- Plasma etching of thin filma.

Dr. Harold BASSECHES

Head, Integrated Circuit Technology Dept.

Bell Telephone Labs.

555 Union Boulevard, Allentown, PA 18104

Iel. : $215-439-7861$

- Deposition of thin metal and dielectric films

for application in the microelectronics tech-

nology.

- Plasma etching techniques.

C.K. BHAT

Head, Materials and Process Engineering Group.

Carnegie-Mellon Institute of Research

4400 Fifth Avenue, Pittsburgh, PA 15213

Te1. : $412-621-1100, \times 215$

- Plasmare heating

- Plasmare melting and refining

- Plasmarc metal consolidation

- Plasmarc remelting
Edwin J. BIELECKI

Manager, Research Chemistry

Kawecki Berylco Industries, Inc.

County Line Road, Boyertown, Pennsylvania 19464

Tel. : 215-367-2181

- Coatings on refractory metals.

- Metal powders.

- Special physical properties of materials.

Dr. Hartwell F. CALCDTE

oirector of Research

Aerochem. Research Laboratories, Inc.

P.0.Box 12, Princeton, N.J.08540

Tel. : 609-921-7070

- Treatment of surfaces

- Chemical synthesis

- Source of ions and excited states of atoma and radicala.

- Environment for studying chemical reactions of ions and excited molecules.

John D. CHASE

Senior Research Engineer

Americal Cyanamid Co.

1937 W. Main St., Stamford, Conn. 06904

Te1. : 203-348-7331

- Fine particle synthesis.

- Ore reduction.

- Pigment synthesis.

J. E. ORUMMOND

0 irector, Plasma Engineering

Maxwell Laboratories, Incorporated

9244 Balboa Ave., San Diego, Ca. 92123

Te1. : 714-279-5100 X134

- Air poliution control.

- Thermonuclear fusion research.

- ore reduction.

Bernard J. EASTLUND

Vice President for Research and Development

Fusion Systems Corporation

11810 Parklawn Dr. Rakville, Md.

Tel. : 301-881-5934

- High power density electrodeless plasmas have been developed for the commercial U.V. polymerizable coating industry.

Hans U. ECKERT

Member of the Technical Staff

The Aerospace Corp. Chemistry and Phys.Lab.

P.D. Box 92957, Los Angeles, Calif. 90009

Tel. : 213-648-6975

- Lasers

- Chemical processing lespecially coal gasifica-

tion)

- Atmospheric reentry

- Astrophysica. 
Maurice G. FEY

Supervising Engineer - Arc Heater Project

Westinghouse Electric Corporation

Trafford, Pennayluania 15085

Tel. : 412-256-5210

- Minerals processing - Ore reduction

- Inorganic chemical production

- Hydrocarbon proceasing

- Procesaes which have potential for oonoumption

of large quantities of electric power.

Dr. Gino J, FRISONE

Sect.Head Analytical Research and Services

The General Tire and Rubber Co.

$R$ and D Center, Akron, Ohio

Te1. : $216-298-2870$

- Surface treatment of plastic and rubber.

W.M. GOLDBERGER

Manager, Mineral and Metallurgical Processing

Battelle-Columbus Laboratories

505, King Avenue, Columbus, Ohio 43201

Tel. : 614-299-3151

- Chemical prooessing, coating, apheroidizing, extractive metallurgy, welding, thermal analyaia, material development.

Or. C.L.GRANT

Associate Oizector, CIID and Profesoor of Chemistry.

Center for Induatrial and Inatitutional Development.

University of New Hampshire, Kingsburv Hall

Durham, New Hampshire 03824

Tel. : 603-862-1354

- processing of metallurgically important orea

to recover values.

- Synthesia of inorganic compounda.

- Sources for analytical spectroscopy.

William R. HARSHBARGER

Member of the Technical Staff

Bell Telephone Laboratories

555 Union Blvd, Allentown, PA 18103

Tel. : 215-439-7844

- Etching silicon, oilicon nitride, oilicon dio-

xide, chromium.

- Stripping photoresiat.

- Depoaiting polymer filmo and thin filma of ailicon nitride.

John C. HAYGARTH

Senior Research Scientist

Teledyne Wah Chang Albany

Box 460, Albany, Oregon 97321

Tel. : 503-926-4211

- Ore treatment

- Preparation of refractory matarialo.

- Coating.
Or. John R. HOLLAHAN

Vice-Preaident, Research and Development

Tegal Corporation

860 Wharf St., Richmond, Calif. 94804

Tel. : 415-232-1757

- Applioations to semiconduotor materials letohing.

oxidation, etc....)

- polymer aurface treatment.

- Thin film depoaition

- Analytical applicationa.

Richard J. JANOWIECKI

Research Group Leader

Monsanto Resesrch Corp.

1515 Nicholas Rd., Oayton, Ohio 45407

Tel. : 513-268-3411 X 315

- Metal, ceramic, polymeric coatings and struotures.

Catherina C. JOHNSON

Chemist

National Aeronatics and Space Administration,

Ames Research Center.

Moffett Field, Calif.

Tel, : 415-965-5723

- plama polymeriaed thin filma for reverae oamosis membranes and plasma polymeriaed thin filmo for optioal coatings, ie antirefleotion and moisture reaistance.

Rdward 0, KAZIMIR

Scientist

NL Industries CRL

Box 420, Hightstown, N.J.

Tel. : 609-448-3200

- procesaing of ilmenite and siroon ores on an industrial scale.

Jed KELLER

Senior Chemiot

Motorola Semiconductor Products Oivision

5005 E Mcoowell Road, PHX, AZ 85008

Tel : 602-244-6843

- The uae of low temperature plama for material processing in semiconductor manufaoturing. Our primary uses for these plasmas are the ashing of organic films and the deposition and etohing of thin filma.

Virgil B. KURFMAN

Research Specialist

Dow Chemical Co.. Central Research Inorganic

Bldg 1703, Midland, MI 48640

Tel. : 517-636-2951

- plasma activation of polymer ourfacea for aubaequent treatmenta, plasma torch coating of various aubatratea. 
Kyle E. LEMONS

Mgr., Materials Res.

Signetics Corp.

811 E Argnes Ave., Sunnyvale, Ca. 94086

Tel. : 408 - 739-7700 × 2733

- Low temperature chemical reactions involving

the manufacture of semiconductors.

Dr. R.LIEPINS

Senior Chemist

Camille Dreyfus Lab., Research Triangle Insticute

P.O.Box 12194, Res.Triangle Park, N.C. 27709

Tel. : 919-549-8311

- Lo- pressure plasma fluidized beds.

- Contiruous lö pressure plasma fiber treatment

$\therefore a c i z i t y$.

Paul M. MORAN

Sr. Electronic Engineer

Instrumentation Lab. Inc. A. I.D.Div.

113, Hartwell Ave., Lexington, Mass.

Tel. : 861-0710 Ext. 384

- se oj R.E.plasma in analytical instrumenta-

tion.

Dr. A.C. MORGAN

Pilot Plant Manager

Cabot Corporation

Concord Rd., Billerica, Mass. 01821

Tel. : 617-272-3500

- Carbon black and other flame process pigment

marufacture.

Dr. F.A.J. Moss

Director, Eastern Research Center

Stauffer Chemical Company

Dobbs Ferry, New York 10522

Te1. : 914-693-1200

Dr. John H. MULLEN

Scientist

McDonnel Oouglas Research Laboratory

Box 516, St.Louis, Mo. 63166

Tel. : 314-232-4687

- Electron attachment.

- Ion production.

- Plasma generation.

- Diagnostica.

- Plasma chemistry.

Daniel O'HARA

Mgr.- Technical Services

Thermal Dynamics Corporation

W. Lebanon, N.H. 03784

Te1. : 603-298-57।1

- Transferred plasma arc torches for welding

and cutting metals.
DI, J.P.REDMOND

Research Associate

AMP lne.

P.0.Box 3608, Harrisburg, PA 17105

Tel. : 717-564-0100 × 1365

- Synthesis of coatings (organic).

Alan R. REINBERG

Member of Technical Staff, Central Research Lab.

Texas Instruments lnc.

P.0.Box 5936, Dallas, Texas 75222

Tel. : $214-238-4466$

- Low pressure deposition of inorganic films.

S. B. ROBOFF

Director, Corporate Development

Kawecki Berylco Industries

220 East 42 nd Street, New-York, N.Y. 10017

Te1. : 212-682-7143

- Plasma spray of materials.

Joseph R. SAGMULLER

Research Scientist

Batcelle Memorial Institute

505 King Ave., Columbus, Ohio.

Tel. : 614-299-3151 ext. 2931

- Plasma sputtering

- Plasma spheroidization

- Microwave plasmas

- Vapor ceposition

Robert J. SCHWAR2

'Sr. Research Physicist

Hercules Incorporated

Research Center, Wilmington, Delaware

Tel. : 302-995-3528

- Clow discharge polymerization of organic compounds.

- Surface modification of polymer fibers and

films by inert and reactive gaseous discharges.

- Hydrogenation and oxidation of organies by

glow discharge.

Michael M. SHAHIN

Manager, Webster Research Center

Xerox Corporation

Rochester, New-York

Tel. : 716-872-2000 ext.22011

- Plasma physics.

- Plasma chemistry.

- Plasma processing.

Dr. Gerald SMOLINSKY

Member of Technical Staff

Bell Telephone Lab. Inc.

Murray Hill, N.J. 07974

Te1. : 201-582-4856

- Polumer deposition. 


\section{STAND}

Director of Resesrch

Sealectro Corp.

225 Hoyd St., Msmaroneck, N.Y. 10543

Tel. : $914-698-5600$

- Spray coating with any powder coating materials, various polymers and plastic composites.

John M. STENGREVICS

Assistant Product Manager

L.F.E. Corporation, Process Control Division

1601 Trapelo Road, Waltham, Mass. 02154

Tel. : 617-890-2000

- Low temperature ashing.

- Plasma cleaning.

- Etching semiconductors and metals.

- Photoresist removal.

- Polymer surface treatment.

Charles $S$. STOKES

Vice-President, Manager Test site

Germantown Laboratories, Inc.

P. O.Box 164, Elverson, PA 19520

Tel. : $215-286-5108$

- Nitrogen fixation.

- Ore melting.

- Chemical reactions.

Dr. William R. STOWELL

Research Scientist

Battelle Columbus Laboratories

505 King Ave., Colombus, Ohio 43220

Tel, : 614-299-3151 ext.3302

- Reactive chemistry.

- Materials spheroidization.

- Sputtering

- Ion plating

- Materials processing using plasmas le.g. plasma spray coatings).

Norman W. THOMAS

Research Associate

Celanese Research Co.

P. O. Box 1000, Summit, N.J.09901

Tel. : 201-469-1504

- Modification of polymers.

- Modification of reinforcements for reinforced

plastios.

Larry THOMPSON

Member of Technical Staff

Bel1 Telephone Labs.

600 Mountain Ave., Murray Hill, N.J. 07933

Tel. : 201-582-2252

- R.F. Plaama $\left(\mathrm{O}_{2}\right)$ cleaning.

- R.F. Plasma etching.

- Plasma polymerization.
Dr. Joseph L. WALDMAN

Associate

N. L. Industries, Titanium Pigment Division

P.O. Box 58, South Amboy, N.J. 08879

Tel. : 201-721-1500 ext. 386

- Industrial application of plasma techniques to inorganic chemical reactions.

- Use of plasma in the production of titanium dioxide pigment.

Dr. Theodore WYDEVEN

Research Scientist

National Aeronatics and Space Administration,

Ames Research Center. Mail Stop N 239-4

Moffett Field, Califoraia 94035

Tel. : 415-965-5738

- Synthesis of semi-permeable membranes for

water purification by plasma polymeriation.

- Deposition of anti-refleotion and moisture resistant coatings for optical components by plasma polymerization.

\section{SWITZERLAND}

Dr. Werner J. BORER

Group Leader

Alusuisse Forschung and Entwicklung

$\mathrm{CH}-8212$ Neuhausen

Tel. : Us3-20221 ext.424

- Plasma apraying.

- Developmant of plasma chemical producto.

Jacques GILLOT

Hesd, Carbons and Inorganic Materials Group

Battelle Geneva Research Center

7. route de Drize, 1227 Carouge/Genève

Tel. : $022 / 439831$

- Inorganic synthesis and especially oynthesis of ultra-fine powders of refractory compounds (carbides, nitrides, oxides, etc...)

\section{Michel KORNMANN}

Chercheur

8attelle Genevo Research Center

7, route de Drize, 1227 Carouge/Genève

Tel. : $022 / 439811$

- sputtering and ion plating.

K. RAGALLER

Head Plasma Physics Group

Brown Boveri Research Center

$\mathrm{CH}-5401$ Baden

Tel.: $056 / 848170$

- High power plasma burners 
1. Bridges, J. M. and 0tt, W. R., The argon mini-arc as a secondary standard of vuv spectral radiance, J. Opt. Soc. Am. 66, 1097 (1976).

2. Bridges, J. M. and Ott, W. R., Vacuum ultraviolet radiometry. 3: The argon mini-arc as a new secondary standard of spectral radiance, Appl. Opt. 16, 367-376 (1977).

3. Canfield, L. R., Johnson, R. G., and Madden, R. P., NBS detector standards for the far ultraviolet, Appl. Opt. 12, 1611-1617 (1973).

4. Ederer, D. L. and Madden, R. P., Use of synchrotron radiation as a known source for spectrometer calibrations, J. Opt. Soc. Am. 64, No. 4, 545 (1974).

5. Ederer, D. L. and Dhez, P., Some applications of GM counters in the vacuum ultraviolet spectral region, Rev. Sci. Instrum. 46, 144-146 (1975).

6. Ederer, D. L., Saloman, E. B., Ebner, S., and Madden, R. P., The use of synchrotron radiation as an absolute source of vuv radiation, J. Res. Nat7. Bur. Stand. (U.S.A.), 79A, 761-774 (1975).

7. Madden, R. P., Synchrotron radiation and applications, X-ray Spectroscopy, Chap. 7, Ed. L. Azaroff, pp. 338-378 (McGraw-Hill Book Co., Inc., New York, 1974).

8. Madden, R. P. and Ederer, D. L., SURF I I, a new synchrotron ultraviolet radiation facility at NBS, Bul1. Amer. Phys. Soc. 19, No. 4, 469 (1974).

9. Ott, W. R., Fieffe-Prevost, P., and Wiese, W. L., A new radiation standard for the vacuum ultraviolet, Bul1. Amer. Phys. Soc. 17, No. 3, 388 (1972).

10. Ott. W. R. and Bartoe, J. D., A recalibration of spectral radiance of mercury and deuterium arc standard lamps in the near uv, J. Opt. Soc. Am. 62, No. 11, 1372 (1972).

11. Ott, W. R., Fieffe-Prevost, P., and Wiese, W. L., VUV radiometry with hydrogen arcs. 1: Principle of the method and comparison with blackbody calibrations from $165 \mathrm{~nm}$ to $360 \mathrm{~nm}$, Appl. Opt. 12, 1618-1629 (1973).

12. Ott, W. R. and Wiese, $\bar{W}$. L., Far ultraviolet spectral radiance calibrations at NBS, Opt. Eng. 12, 86-94 (1973).

13. Ott, W. R. and Wiese, W. L., Hydrogen arc radiometry applied to fusion effort, Nat. Bur. Stand. (U.S.A.), Tech. News. Bul1. 57, No. 6, 144-145 (1973).

14. Ott, W. R. and Gieres, G., A new transfer standard for vuv spectral radiance calibrations, J. Opt. Soc. Am. 64, 1405 (1974).

15. Ott, W. R. and Gieres, G., An improved method for vuv radiometric calibrations using hydrogen arcs, Bu11. Amer. Phys. Soc. 20, 248 (1975).

16. Ott, W. R., Behringer, K., and Gieres, G., VUV radiometry with hydrogen arcs. 2: The high power arc as an absolute standard of spectral radiance from $124 \mathrm{~nm}$ to $360 \mathrm{~nm}$, Appl. Opt. 14, 2121-2128 (1975).

17. Ott, W. R., NBS ultraviolet radiometric standards, Symposium on Measurements for the Safe Use of Radiation, Gaithersburg, Md., March, 1976. NBS-SP456, 107-110 (1976).

18. Saloman, E. B., Ederer, D. L., and Madden, R. P., Windowless diode radiometric transfer standards from 20 to $120 \mathrm{~nm}$, J. Opt. Soc. Am. 64, No. 4, 547 (1974).

19. Saloman, E. B. and Ederer, D. L., Absolute radiometric calibration of detectors between 20-60 nm, Appl. Opt. 14, 1029-1034 (1975).

20. Saloman, E. B., Unfolding first and second order diffracted radiation when using synchrotron radiation sources: A technique, Appl. Opt. 14, 1391-1394 (1975).

21. Saloman, E. B., Time response of NBS windowless xuv radiometric transfer standard detectors, Appi. Opt. 14, 1764 (1975).

22. Saunders, R. D. and ott, W. R., Spectral irradiance measurements: Effect of uv-produced fluorescence using integrating spheres, Appl. 0pt. 15, 827 (1976).

23. Saunders, R. D., Ott, W. R., and Bridges, J. M., Spectral irradiance standard for the ultraviolet, J. Opt. Soc. Am. 66, 1097 (1976). 
1. Ederer, D. L., Use of synchrotron for radiometry--progress report, 6 th Synchrotron Users Meeting, Stoughton, Wisc., Oct. 1973.

2. Ederer, D. L., Synchrotron radiation as an absolute radiometric standard as applied to SURF-II, 3rd Workshop on VUV Radiometric Calibrations of Space Experiments, Boulder, Colo., Sept. 1974.

3. Madden R. P. and Ederer, D. L., Transfer standard detectors and synchrotron calibrations, lst Workshop on VUV Radiometric Calibrations of Space Experiments, Gaithersburg, Md., Nov. 1971.

4. Madden, R. P., Detector standards and synchrotron radiation for vuv calibrations, NBS Astronomy Day Program, Feb. 18, 1972.

5. Madden, R. P., Synchrotron radiation and applications, Optical Sciences Center Colloquium, University of Arizona, Tucson, Mar. 1972.

6. Madden, R. P., Synchrotron radiation and applications, Physics Department Colloquium, University of Virginia, Mar. 1972.

7. Madden, R. P. and Saloman, E. B., Photodiode standard detectors 20-254 nm, 2nd Workshop on VUV Radiometric Calibrations of Space Experiments, Boulder, Colo., Nov. 1972.

8. Madden, R. P., Contamination of tungsten photocathodes, 2nd Workshop on VUV Radiometric Calibrations of Space Experiments, Boulder, Colo., Nov. 1972.

9. Madden, R. P., Far uv radiometric activities at NBS, 3rd Workshop on VUV Radiometric Calibrations of Space Experiments, Boulder, Colo., Sept. 1974.

10. Madden, R. P., Far uv radiometric standards, NBS Workshop on Atomic Data for Fusion, Gaithersburg, Md., Mar. 1975.

11. Madden, R. P., The status of SURF II as a radiometric standard, 4th Workshop on VUV Radiometric Calibrations of Space Experiments, Boulder, Colo., April, 1976.

12. Madden, R. P., Radiometry with synchrotron radiation, Synchrotron Radiation Facilities Quebec Summer Workshop, June, 1977.

13. Ott, W. R. and Wiese, W. L., The hydrogen arc continuum as a calibration source, 1st Workshop on VUV Radiometric Calibrations of Space Experiments, Gaithersburg, Md., Nov. 1971.

14. Ott, W. R., The wall-stabilized hydrogen arc as an absolute standard for the vuv, NBS Astronomy Day Program, Feb. 18, 1972.

15. Ott, W. R., Progress on the NBS wall-stabilized hydrogen arc, 2nd Workshop on VUV Radiometric Calibrations of Space Experiments, Boulder, Colo., Nov. 1972.

16. Ott, W. R., Vacuum ultraviolet radiometry at NBS, Physikalische Technische Bundesanstalt Colloquium, Berlin, Germany, Sept. 1973.

17. Ott, W. R., Absolute vuv radiometry with hydrogen arcs--comparisons with blackbody calibrations, 11 th International Conference on Phenomena in Ionized Gases, Prague, Czechoslovakia, Sept. .1973.

18. Ott, W. R., A new transfer standard for vuv spectral radiance calibrations, IAU Colloquium No. 27, 4th Conference on UV and X-Ray Spectroscopy of Astrophysics and Laboratory Plasma, Sept. 9-11, 1974.

19. Ott, W. R., NBS research on ultraviolet transfer source standards of spectral radiance and irradiance, 3rd Workshop on VUV Radiometric Calibrations of Space Experiments, Boulder, Colo., Sept. 1974.

20. Ott, W. R., Review of present standard source technology, 4th Workshop on VUV Radiometric Calibrations of Space Experiments, Boulder, Colo., April, 1976.

21. Ott, W. R., The argon mini-arc as a new transfer standard of spectral radiance in the far ultraviolet, 4 th Workshop on VUV Radiometric Calibrations of Space Experiments, Boulder, Colo., April, 1976.

22. Ott, W. R., NBS ultraviolet radiometric standards, 12th Informal Conference on Photochemistry, Gaithersburg, Md., June 1976.

23. Ott. W. R., Sources of UV-B radiation, Environmental Protection Agency Biological Instrumentation Workshop, Washington, D.C., November, 1976.

24. Saloman, E. B., Ederer, D. L., and Madden, R. P., Radiometry in the euv spectral region: Standard sources and detectors, 4th International Conference on VUV Radiation Physics, Hamburg, Germany, July 22-26, 1974.

25. Saloman, E. B., Calibration of radiometric standard detectors between 20 and $60 \mathrm{~nm}$, 3rd Workshop on VUV Radiometric Calibrations of Space Experiments, Boulder, Colo., Sept. 1974. 
26. Saloman, E. B., NBS transfer standard diodes, 4 th Workshop on VUV Radiometric Calibrations of Space Experiments, Boulder, Colo., April, 1976.

27. Wiese, W. L., Arc plasmas as radiation standards in the vacuum ultraviolet, 6 th Yugoslav Symposium and Summer School on the Physics of Ionized Gases, Miljevac, Yugoslavia, July 1972.

28. Wiese, $W$. L., The high current hydrogen arc--a new radiometric standard source for the vacuum ultraviolet, Culham Laboratories Plasma Physics Colloquium, Abingdon, England, July 1973.

29. Zalewski, E. F., Detectors of UV-B radiation, Environmental Protection Agency Biological Instrumentation Workshop, Washington, D.C., November, 1976. 


\section{REFERENCES}

1. Criteria for a recommended standard...occupational exposure to ultraviolet radiation, U.S. Department of Hea1th, Education, and Welfare, Report HSM73-11009, 96 pages (1972).

2. Steiner, B., The Present State of Radiometry and Photometry, Nat. Bur. Stand. (U.S.), Tech. Note 594-6, 56 pages (Mar. 1974).

3. Electro-Optical Systems Design 5, No. 12, 11-29 (Nov. 1973).

4. Wright, A. N., Ultraviolet sources for photochemical application, Polymer Eng. \& Sci. 11 , No. 5, 416-420 (1971).

5. Hagen, W. F., Ultraviolet lasers, Industrial Research 14, No. 5, 48-51(1972).

6. Rhodes, C. K., Review of ultraviolet laser physics, IEEE J. Quantum Electron. QE-10, No. 2, 154-174 (Feb. 1974).

7. Elton, R. C., Waynant, R. W., Andrews, R. A., and Reilly, M. H., X-ray and vacuum uv lasers: current status and prognosis, Naval Research Laboratory Report 7412, 23 pages (May 1972).

8. Kostkowski, H. J., Erminy, D. E., and Hattenberg, A. T., High accuracy spectral radiance calibrations of tungsten strip lamps, Chap. 4, Advances in Geophysics, pp. 111-127 (Academic Press, Inc., New York, 1970).

9. Goldbach, C., Nollez, G., and Peyturaux, R., A study of a high-pressure thermal argon plasma as a high-radiance standard, J. Quant. Spectrosc. Radiat. Transfer 12, No.7, 1089-1104 (1972).

10. Boldt, G., The thermal plasma as a standard intensity source in the wavelength range from 110 to $310 \mathrm{~nm}$, Space Sci. Rev. 11, 728-772 (1970).

11. Ott, W. R., Behringer, K., and Gieres, G., VUV radiometry with hydrogen arcs. 2. The high power arc as an absolute standard of spectral radiance from $124 \mathrm{~nm}$ to $360 \mathrm{~nm}$, App 1. Opt. 14, 2121-2128 (1975).

12. Codling, K. and Madden, R. P., Characteristics of the synchrotron light from the NBS $180 \mathrm{MeV}$ machine, J. Appl. Phys. 36, No. 2, 380-387 (1965).

13. Ederer, D. L., Saloman, E. B., Ebner, S., and Madden, R. P., The use of synchrotron radiation as an absolute source of vuv radiation, J. Res. Nat1. Bur. Stand. (U.S.A.), 79A, 761-774 (1975).

14. Ott, W. R. and Wiese, W. L., Far ultraviolet spectral radiance calibrations at NBS, Opt. Eng. 12, 86-94 (1973).

15. Pitz, E., Absolute calibration of light sources in the vacuum ultraviolet by means of the synchrotron radiation of DESY, Appl. Opt. 8 , No. 2, 255-259 (1969).

16. Optical Radiation News, National Bureau of Standards, 14, 1 (1976).

17. Hattenburg, A. T., Spectral radiance of a low current graphite arc, App1. Opt. $\underline{6}$, 95-100 (1967).

18. Hua, C. T., Cerenkov radiation as a standard source for rocket experiments: measurement of the spectral energy distribution. Astron. Astrophys. 27, 255-260 (1973).

19. Geist, J. and Blevin, W. R., Chopper-stabilized null radiometer based upon an electrically calibrated pyroelectric detector, Appl. Opt. 12, No. 11, 2532-2535 (1973).

20. Samson, J. A. R., Techniques of Vacuum U1traviolet Spectroscopy, pp . 265-277 (John Wiley and Sons, Inc., New York, 1967).

21. Canfield, L. R., Johnson, R. G., and Madden, R. P., NBS detector standard for the far ultraviolet, Appl. Opt. 12, No. 7, 1611-1617 (1973).

22. Hudson, R. D., National Bureau of Standards, private communication.

23. Miles, B. M. and Wiese, W. L., Bibliography on Atomic Transition Probabilities, Nat. Bur. Stand. (U.S.), Spec. Pub1. 320, 111 pages (Feb. 1970).

24. Wiese, W. L., Smith, M. W., and Glennon, B. M., Atomic Transition Probabilities, Hydrogen through Neon, Vol. I, Nat. Stand. Ref. Data Ser., Nat. Bur. Stand. (U.S.), 4, 153 pages (May 1966). Also, Sodium through Calcium, Vol. II, Nat. Stand. Ref. Data Ser., Nat. Bur. Stand. (U.S.), 22, 268 pages (Oct. 1969).

25. Hinnov, E. and Hofmann, F. W., Measurement of absolute radiation intensities in the vacuum ultraviolet region, J. Opt. Soc. Am. 53, No. 11, 1259-1265 (1963).

26. Magdeburg, H., Spektraler Emissionsgrad und Strah̄idichte des Niederstrom-Kohlebogens, Z. Naturforsch. A 20,980-981 (1965).

27. Pitz, E., Spectral radiance of the carbon arc between $250 \mathrm{~nm}$ and $190 \mathrm{~nm}$, App1. 0pt. 10, No. 4, 813-818 (1971).

28. Stuck, D. and Wende, B., Photometric comparison between two calculable vacuum ultraviolet standard radiation sources: synchrotron radiation and plasma blackbody radiation, J. Opt. Soc. Am. 62, No. 1, 96-100 (1972). 
29. Pitz, E., private communication.

30. Hessburg, H., Niekerke, J., and Stephan, K.H., Absolute rocket photometry of $\gamma$-Ori in the vuv region, Astron. and Astrophys. 42, 395 (1975).

31. Ott, W. R. and Bartoe, J. D., A recalibration of spectral radiance of mercury and deuterium arc standard lamps in the near uv, J. Opt. Soc. Am. 62, No. 11, 1372 (1972).

32. Nakagawa, Y. and Otani, F., The suitability of deuterium lamps as a short ultraviolet spectral irradiance standard, Tokyo J. of the I11um. Eng. Inst. of Japan 56, No. 12, 678-681 (1972).

33. Guenther, K. and Radtke, R., Radiation standard for the visible and near uv region, Proc. 11th Int1. Conf. Phen. in Ion. Gases (Prague, Czechoslovakia, Sept. 10-14, 1973), p. 446.

34. Fisher, G. B., Spicer, W. E., McKernan, P. C., Pereskok, V. F., and Wanner, S. J., A standard for ultraviolet radiation, App1. Opt. 12, No. 4, 799-804 (1973).

35. Minutes of the second workshop on the vuv radiometric calibrations of space experiments, Boulder, Colorado, Nov. 28-29, 1972, available from J. L. Linsky, JILA, Boulder, Colo.

36. Physics Today 28, No. 3, 74 (Mar. 1975).

37. Maylotte, D. H. and Wright, A. N., Surface photopolymerization from tetrafluorethylene, General Electric Tech. Inf. Ser., Report No. 74CRD170, 18 pages (Aug. 1974).

38. Radiation and the textile industry, Optical Spectra 8, No. 10, 15 (0ct. 1974).

39. Schroeter, S. H. and lloore, J. E., The ultraviolet cure of solventless resins--a pollution free method?, Nonpolluting Coatings and Coating Processes, Ed. J. L. Gardon and J. W. Prane, pp. 135-147 (Plenum Publishing Corp., New York 1973).

40. Schroeter, S. H., Moore, J. E., and Orkin, O. V., The ultraviolet cure of unsaturated polyestermonomer systems, Coatings and Plastics Preprints 34, 751-758 (1974).

41. Schroeter, S. H., The ultraviolet curing of coatings, Nonpolituting Coatings and Coating Processes, Ed. J. L. Gardon and J. W. Prane, pp. 109-133 (Plenum Publishing Corp., New York, 1973).

42. Plastic replaces plaster in new casting system, Chemical and Engineering News 51, No. 31,17 (JuTy 30, 1973).

43. The energy crisis--tapping the sun, Optical Spectra 7, No. 3, 27-38 (1973).

44. Hart, D., Bactericidal ultraviolet radiation in the operating room--twenty-nine year study for control of infections, J. Amer. Med. Assoc. 137, 1600-1603 (1948).

45. Hart, D. and Nicks, J., U1traviolet radiation in the operating room--intensities used and bactericidal effects, Arch. Surg. 82, 449-465 (1961).

46. Riley, R. L. and O'Grady, F., Airborne Infection--Transmission and Control, (Macmillan Co., Hew York, 1961).

47. McLean, R. L., The effect of ultraviolet radiation upon the transmission of epidemic influenza in long term hospital patients, Amer. Rev. Resp. Dis. 83, 36-40 (Feb: pt. 2, 1961).

48. Nagy, R., Mouromseff, G., and Rixton, F. H., Disinfecting air with sterilizing lamps, Heating, Piping, Air Cond. 26, 82-87 (1954).

49. Phillips, G. B. and Hanel, Jr., E., Use of ultraviolet radiation in microbiological laboratories, Library of Congress, Photoduplication Services, Publication Board Project PB147043 (1960).

50. Hanson, K. J., Limitation of radiometry to meteorology and oceanography, J. Opt. Soc. Am. 62, $1342(1972)$.

51. Johnston, H., Reduction of stratospheric ozone by nitrogen oxide catalysts from supersonic transport exhaust, Science 173, 517-522 (1971).

52. Crutzen, P. J., The influence of nitrogen oxides on the atmospheric ozone content, Quart. J. Roy. Meteorol. Soc. 96, 320-325 (1970).

53. Heath, D., Some experimental techniques and problems associated with stratospheric measurements (Proc. Survey Conf. Climatic Impact Assess. Prog., Cambridge, Mass., Feb. 15-16, 1972), Dept. of Transp. (U.S.) Report DOT-TSC-0ST-72-13, pp. 226-242.

54. Slavin, W., Spectroscopy in the clinic, Optical Spectra 8, No. 10, 36-39 (1974).

55. Thorington, L., Cunningham, L., and Parascandola, J., The $i 17$ uminant in the prevention and phototherapy of hyperbilirubinemia, I1lum. Eng. 66, No. 4, 240-250 (1971).

56. Loomis, W. F., Rickets, Sci. Am. 223, 77-91 (1970).

57. Neer, R. M., Davis, T. A. R., Walcott, A., Koski, S., Schepis, P., Taylor, I., Thorington, L., and Wurtman, R. J., Stimulation by artificial lighting of calcium absorption in elderly human subjects, Nature 229, 255-256 (1971).

58. Neer, R. M., Davis, T., Thorington, L., and Litt Te, A. D., Use of environmental lighting to stimulate calcium absorption in healthy men, Clinical Res. 18, No. 4, $693(1970)$. 
59. Thorington, L., Parascandola, L., and Cunningham, L., Visual and biologic aspects of an artificial sunlight illuminant, J. Illum. Eng. Soc. 1, No. 1, 33-41 (1971).

60. Volkova, N. V., Experience in the use of erythemic uTtraviolet radiation in the general lighting system of a machine shop, Gigiena: Sanitariya (U.S.S.R) 32, 109-111 (Oct. 1967).

61. Zamkova, M. A. and Krivitskaya, E. I., Effect of irradiation by ultraviolet erythema lamps on the working ability of school children, Gigiena: Sanitariya (U.S.S.R.) 31, 41-44 (April 1966).

62. Wurtman, R. J. and Neer, R. M., Good 1ight and bad, New Eng1. J. Med. 282, No. 7, 394-395 (Feb. 12, 1970).

63. Bishop, J. E., New treatment that clears up psoriasis is reported by research team in Boston, Wall Street Journal (Dec. 5, 1974).

64. Parrish, J. A., Fitzpatrick, T. B., Tanenbaum, L., and Pathak, M. A., Photochemotherapy of psoriasis with oral methoxsalen and long wave ultraviolet iight, New Engl. J. Med. 291, No. 23, 1207-1211 (Dec. 5, 1974).

65. UTrich, W. F., Solutions from spectra, Industrial Research 13, No. 6, 52-55 (June 1971).

66. Oke, J. B., Absolute flux measurements in astronomy, J. Opt. Soc. Am. 62, 1342 (1972).

67. Harris, S. E., Kung, A. H., and Young, J. F., Nonlinear optical techniques for the generation of ultraviolet, vacuum ultraviolet, and soft $x$-ray radiation, J. Opt. Soc. Am. 64, No. 4, 556 (1974).

68. A laser promise, Optical Spectra 8, No. 7, 12 (July 1974).

69. Light processes are seen to enrich heavy isotopes, Industrial Research 16, No. 8, 19-21 (Aug. 1974).

70. Souping up electrons for lasers and fusion, Industrial Research 15, No. 9, 30-32 (Sept. 1973).

71. LaVilia, R. E., The sulfur $K$ and $L$ and fluorine $K X$-ray emission and absorption spectra of gaseous $\mathrm{SF}_{6}$, J. Chem. Phys. 57, 899-909 (1972).

72. Kinsey, V. E., Cogan, D. G., and Drinker, P., Measuring eye flash from arc welding, J. Amer. Med. Assoc. 123, 403-404 (1943).

73. Rieke, F. E., Arc flash conjunctivitis: actinic conjunctivitis from electric welding arc, J. Amer. Med. Assoc. 122, 734-736 (1943).

74. Grim, R. C. and Kusnetz, H. L., The plasma torch--industrial hygiene aspects, Arch. Environ. Health 4, 295-400 (1962).

75. Powell, C. H., Goldman, L., and Key, M. M., Investigative studies of plasma torch hazards, Amer. Ind. Hyg. Assoc. J. 29, 381-385 (1968).

76. Kinsey, V. E., Spectral transmission of the eye to ultraviolet radiations, Arch. Ophthalmol. 39, 508-513 (1948).

77. Bachem, A., Ophthalmic ultraviolet action spectra, Am. J. Ophthalmo1. 41, 969-975 (1956).

78. Cogan, D. G. and Kinsey, V. E., Action Spectra of keratitis produced by ultraviolet radiation, Arch. Ophthalmol. 35, 670-677 (1946).

79. Sherashov, S. G. , Spectral sensitivity of the cornea to ultraviolet radiation, Biofisika (U.S.S.R.) 15, 569-571 (1970).

80. James, A. P. R., Sensitivity of the skin to fluorescent 1ight, Arch. Dermatol. 44, 256-257 (1941).

81 Bresler, R. R., Cutaneous burns due to fluorescent 1ight, J. Amer. Med. Assoc. 140, 1334-1336 (1949).

82. Pathak, M. A., Basic aspects of cutaneous photosensitization, The Biologic Effects of Ultraviolet Radiation with Emphasis on the Skin, Ed. F. Urbach, pp. 489-511

(Pergamon Press, New York,' 1969).

83. Gorter, E., On rickets, J. Pediat. 4, 1-11 (1934).

84. Coblentz, W. W., Stair, R., and Hogue, J. M., Spectral erythemic reaction of the untanned human skin to ul traviolet radiation, J. Res. Nat. Bur. Stand. (U.S.), $\underline{8}$, 541-547 (1932).

85. Epstein, J. H., Ultraviolet light carcinogenesis, Advances in Biology of Skin, pp. 215-236 (Pergamon Press, New York, 1969).

86. Epstein, J. H., Epstein, W. L., and Nakai, J., Production of melanomas in hairless mice with ultraviolet light, Ciinical Res. 13, 226 (1965).

87. Lancaster, H. O. and Nelson, J., Sunlight as a cause of melanoma: a clinical survey, Med. J. Aust. I, 452-456 (1957).

88. Blum, H. F., Quantitative aspects of cancer induction by ultraviolet light: including a revised model, The Biologic Effects of Ultraviolet Radiation with Emphasis on the Skin, Ed. F. Urbach, pp. 543-549 (Pergamon Press, New York, 1969). 
89. Blum, H. F., Ultraviolet light and skin cancer, Proc. XII Int1. Congr. Dermatol. Excerpt Medica Int1. Congr. Series No. 55, pp. 296-301 (1962).

90. Brodkin, R. H., Kopf, A. W., and Andrade, R., Basal-cell epithelioma and elastosis: a comparison of distribution. The Biologic Effects of U1traviolet Radiation with Emphasis on the Skin, Ed. F. Urbach, pp. 581-618 (Pergamon Press, New York, 1969).

91. Blum, H. F., Carcinogenes is by U1traviolet Light, 340 pages (Princeton University Press, Princeton, New Jersey, 1959).

92. Urbach, F., Geographic pathology of skin cancer, The Biologic Effects of U1traviolet Radiation with Emphasis on the Skin, Ed. F. Urbach, pp. 635-650 (Pergamon Press, New York, 1969).

93. Urbach, F., Davies, R. E., and Forbes, P. D., U1traviolet radiation and skin cancer in man, Advances in Biology of Skin, Vol. XII, pp. 195-214 (Pergamon Press, New York, 1966).

94. Mika, L. A., Officer, J. E., and Brown, A., Inactivation of two arboviruses and their associated infectious nucleic acids, J. Infect. Dis. 113, 195-203 (1963).

95. Swenson, P. A. and Swetlow, R. B., B-Galactosidase: inactivation of its messenger RNA by ultraviolet irradiation, Science 146, 791-793 (1964).

96. Freeman, R. C., Hudson, H. T., and Carnes, R., U1traviolet wavelength factors in solar radiation and skin cancer, Int1. J. Dermato1. 9, 232-235 (1970).

97. MacDona1d, E. J., The epidemiology of skin cancer, J. Invest. Dermato1. 32, 379-382 (1959).

98. Kligman, A. M., Early destructive effect of sunlight on human skin, J. Amer. Med. Assoc. 210, 2377-2380 (1969).

99. Papa, C.M. and K1igman, A. M., The effect of topical steroids on the aged human axilla, Advances in Biology of Skin, Ed. W. Montagna, Vol. XI, pp. 165-198 (Pergamon Press, New York, 1965).

100. Smith, J. G. and Lansing, A. I., Distribution of solar elastosis (senite elastosis) in the skin, J. Geronto1. 14, 496 (1959).

101. Suntamp-related injuries analys is for FY 1973 and FY 1974, ACPE Study 9, available from Medical Data Systems Staff, FDA Office of Planning and Evaluation (HFP-50), Rockville, Md.

102. Barnes, R., An unusual hazard in forgery detection, Med. J. Aust. 1, 540-541 (1970).

103. Nagy, R., Application of ozone from sterilamp in control of mold, bacteria, and odors, Advances in Chemistry Series No. 21, pp. 57-65 (American Chemical Society, 1959).

104. Nagy, R., Application and measurement of ultraviolet radiation, Ind. Hyg. J. 25, 274-281 (1964).

105. Gafafer, W. M., Occupational diseases--a guide to their recognition, Dept. of Health, Education, and Welfare (U.S.), Public Health Service, Publ. 1097, pp. 260-261 (1966).

106. Work on X-ray laser continues, Electro-Optical Systems Design $\underline{5}$, No. 13, 12 (Dec. 1973).

107. Nature 252, No. 5482, Cover 3 (Nov. 29, 1974).

108. Böhm, W. and Labs, D., Transition radiation as a secondary standard source in the vuv, App 1. Opt. 10, 2021-2023 (1971).

109. U.P.I., Dentists told to halt use of 1 ight devices, Washington Post, May 23, 1975. 

NBS.114A (REV. 7.73)

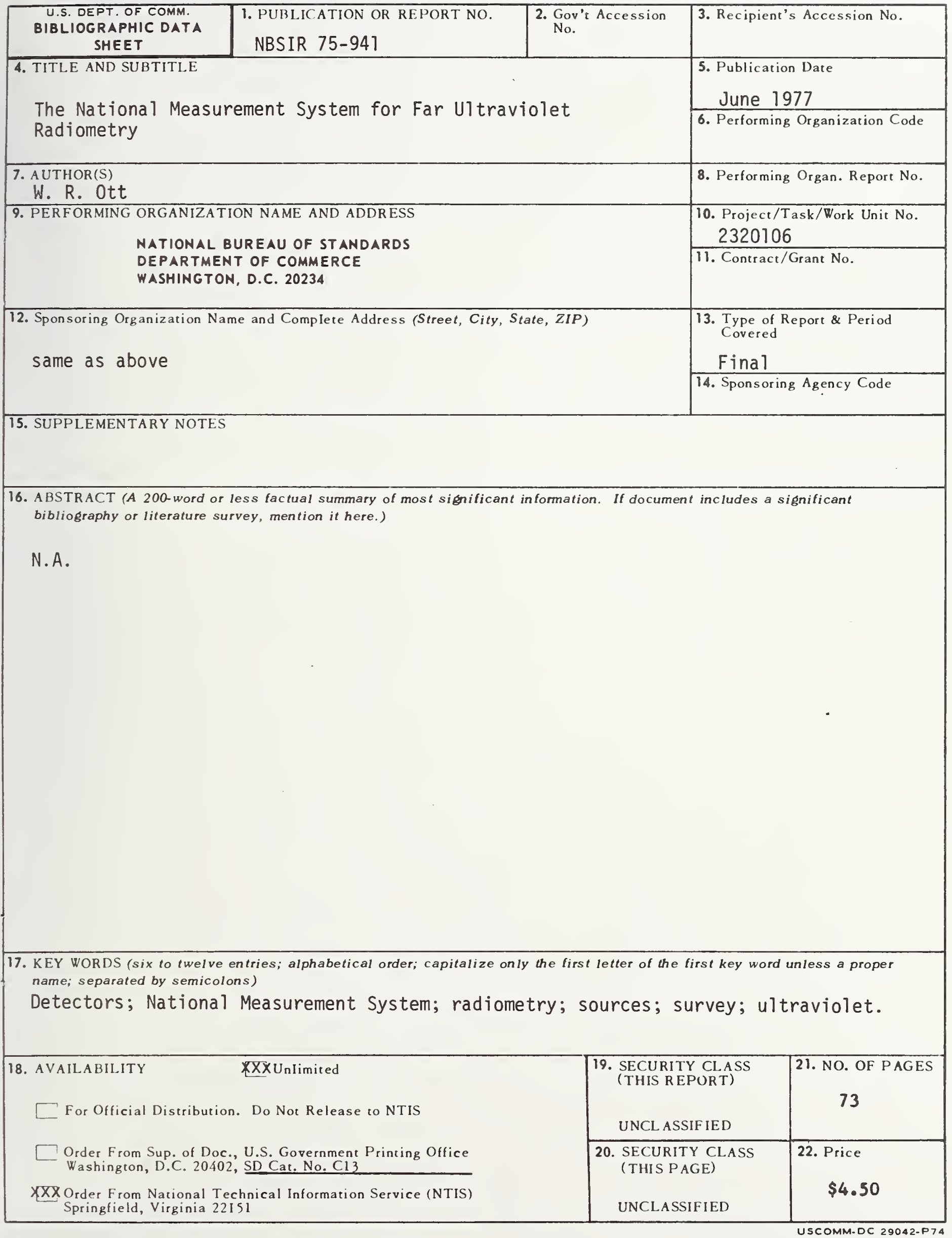




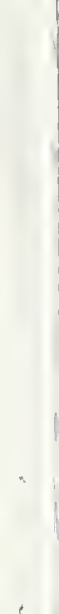



